\title{
A Convergent Synthesis of Polysubstituted Aromatic Nitriles via Palladium Catalyzed C-H Functionalization
}

\author{
Brian Mariampillai, Julien Alliot, Mengzhou Li and Mark Lautens* \\ Davenport Research Laboratories, Department of Chemistry, University of Toronto, Toronto, \\ Ontario, Canada M5S 3 H6
}

\section{SUPPORTING INFORMATION}

General Experimental

Intermolecular Alkylation/Cyanation Reaction:

Intermolecular Alkylation/Cyanation Reaction Using Alkyl Chlorides:

Intermolecular Arylation/Cyanation Reaction Using Aryl Bromides: 


\section{General Experimental}

Infrared (IR) spectra were recorded on a Perkin Elmer FT-IR spectrometer, SPECTRUM 1000. ${ }^{1} \mathrm{H}$ and ${ }^{13} \mathrm{C}$ NMR spectra were measured on Varian Unity 500, Varian Mercury 400, Varian 400 and Varian Mercury 300 spectrometers. Splitting patterns are indicated as s, singlet; d, doublet; t, triplet; q, quartet; m, multiplet. Melting points were carried out on a Fisher-Johns Melting Point Apparatus, all solids were crystallized from dichloromethane via slow evaporation. For thin layer chromatography (TLC) analysis throughout this work, Silicycle precoated TLC plates (silica gel $60 \mathrm{~F}_{254}$ on aluminum sheet) were used. The products were purified by preparative column chromatography on silica gel $(60 \AA$, ultrapure silica gel, SILICYCLE). The high-resolution mass spectra (HRMS) analysis was performed at the Department of Chemistry, University of Toronto (EI and ESI). Dimethoxyethane and acetonitrile were distilled from calcium hydride stills under nitrogen prior to use. Potassium hexacyanoferrate trihydrate was ground to a fine powder using mortar and pestle. Anhydrous potassium hexacyanoferrate was prepared according to the procedure of Beller. ${ }^{1}$ Iodonaphthalene was purified via column chromatography prior to use, using hexane as eluent; all other starting materials were purchased from Aldrich, VWR and Strem Chemicals and used as received. N-(2iodophenyl)-4-N-dimethylbenzenesulfonamide, ${ }^{2} \quad$ N-(3-iodo-2-methylphenyl)-4-Ndimethylbenzenesulfonamide, ${ }^{3}$ and $\mathrm{N}$-(4-iodophenyl)-4-N-dimethylbenzenesulfonamide, ${ }^{2}$ were prepared according to published literature procedures. Reactants were filled into appropriate microwave process vials and either heated in oilbath or using microwave irradiation. Microwave reactions were performed on a Biotage Initiator EXP US System using $5 \mathrm{ml}$ vials and septa purchased from Biotage. For experimental details of intramolecular ortho alkylation/cyanation please see: J. Am. Chem. Soc. 2006, 128, 14436-14437 supporting information.

\footnotetext{
${ }^{1}$ Schareina, T.; Zapf, A.; Maegerlein, W.; Mueller, N.; Beller, M. Tet. Lett. 2007, 48, 1087-1090.

${ }^{2}$ Wilhelm, T.; Lautens, M. Org. Lett. 2005, 7, 4053-4056.

${ }^{3}$ Martins, A.; Alberico, D.; Lautens, M. Org. Lett. 2006, 8, 4827-4829.
} 
General Procedure for Intermolecular Alkylation/Cyanation Reaction: To a $5 \mathrm{ml}$ microwave vial containing a magnetic stir bar was added 1.0 equivalent aryl iodide, 10.0 equivalents alkyl halide, 0.1 equivalents palladium acetate, 0.22 equivalents triphenylphosphine, 4.5 equivalents $\mathrm{K}_{4}\left[\mathrm{Fe}(\mathrm{CN})_{6}\right] 3 \mathrm{H}_{2} \mathrm{O}, 3.0$ equivalents norbornene and 2.0 equivalents cesium carbonate. The vial was then sealed and flushed with argon. Dimethoxyethane was added to give a final concentration of $0.05 \mathrm{M}$ based on the aryl iodide. The resulting solution was then purged with argon for 5 minutes followed by heating with stirring in a preheated oil bath set to $90{ }^{\circ} \mathrm{C}$ for 24 hours. The mixture was then diluted with water and extracted with dichloromethane. The combined organic phases were dried with sodium sulphate and evaporated under reduced pressure. The product was then purified by flash column chromatography.

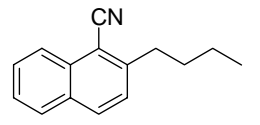

2-Butyl-naphthalene-1-carbonitrile (25): Following the general procedure above on 0.2 mmol of 1-iodonaphthalene and iodobutane as the alkylating agent. The crude product was purified by column chromatography using $20 \%$ dichloromethane in hexane to give $39 \mathrm{mg}\left(91 \%\right.$ Yield) as a clear colorless oil. ${ }^{1} \mathrm{H} \mathrm{NMR}\left(\mathrm{CDCl}_{3}, 300 \mathrm{MHz}\right) \delta 8.22(\mathrm{dd}, 1 \mathrm{H}$, $\mathrm{J}=1.0 \mathrm{~Hz}, \mathrm{~J}=8.4 \mathrm{~Hz}), 7.97(\mathrm{~d}, 1 \mathrm{H}, \mathrm{J}=8.5 \mathrm{~Hz}), 7.87(\mathrm{~d}, 1 \mathrm{H}, \mathrm{J}=8.1 \mathrm{~Hz}), 7.66$ (ddd, $1 \mathrm{H}$, $\mathrm{J}=1.3 \mathrm{~Hz}, \mathrm{~J}=6.9 \mathrm{~Hz}, \mathrm{~J}=8.4 \mathrm{~Hz}), 7.55(\mathrm{ddd}, 1 \mathrm{H}, \mathrm{J}=1.2 \mathrm{~Hz}, \mathrm{~J}=6.9 \mathrm{~Hz}, \mathrm{~J}=8.1 \mathrm{~Hz}), 7.41(\mathrm{~d}, 1 \mathrm{H}$, $\mathrm{J}=8.5 \mathrm{~Hz}), 3.09-3.01(\mathrm{~m}, 2 \mathrm{H}), 1.80-1.68(\mathrm{~m}, 2 \mathrm{H}), 1.51-1.37(\mathrm{~m}, 2 \mathrm{H}), 0.97(\mathrm{t}, 3 \mathrm{H}$, $\mathrm{J}=7.3 \mathrm{~Hz}) ;{ }^{13} \mathrm{C}-\mathrm{NMR}\left(\mathrm{CDCl}_{3}, 75 \mathrm{MHz}\right) \delta 148.0,133.1,132.9,131.5,128.7,128.6,127.1$, 126.8, 125.3, 117.3, 109.1, 35.4, 33.5, 22.6, 14.1; IR (film) 3056, 2957, 2930, 2870, 2362, 2214, 1623, 1594, 1507, 1466, 1377, 1272, 1147, 1094, 1027, 868, 823, $752 \mathrm{~cm}^{-1}$; HRMS-EI (m/z): $\left[\mathrm{M}^{+}\right]$calcd for $\mathrm{C}_{15} \mathrm{H}_{15} \mathrm{~N}$ : 209.1204 found 209.1199 .<smiles>CCCCc1cccc(C)c1C#N</smiles>

2-Butyl-6-methyl-benzonitrile (26): Following the general procedure above on 0.2 mmol of 1-iodo-2-methylbenzene and iodobutane as the alkylating agent. The crude product was purified by column chromatography using $20 \%$ dichloromethane in hexane to give $29 \mathrm{mg}\left(84 \%\right.$ Yield) as a clear colorless oil. ${ }^{1} \mathrm{H} \mathrm{NMR}\left(\mathrm{CDCl}_{3}, 400 \mathrm{MHz}\right) \delta 7.36$ (t, $1 \mathrm{H}, \mathrm{J}=7.7 \mathrm{~Hz}), 7.12(\mathrm{dd}, 2 \mathrm{H}, \mathrm{J}=2.8 \mathrm{~Hz}, \mathrm{~J}=7.7 \mathrm{~Hz}), 2.88-2.79(\mathrm{~m}, 2 \mathrm{H}), 2.54$ (s, $3 \mathrm{H}), 1.71-$ $1.59(\mathrm{~m}, 2 \mathrm{H}), 1.46-1.34(\mathrm{~m}, 2 \mathrm{H}), 0.95(\mathrm{t}, 3 \mathrm{H}, \mathrm{J}=7.3 \mathrm{~Hz}) ;{ }^{13} \mathrm{C}-\mathrm{NMR}\left(\mathrm{CDCl}_{3}, 100 \mathrm{MHz}\right) \delta$ 147.1, 142.2, 132.0, 127.4, 126.5, 117.2, 112.8, 34.6, 33.0, 22.4, 20.8, 13.8; IR (film) 2957, 2866, 2218, 1594, 1468, 1381, 1037, 782, $747 \mathrm{~cm}^{-1}$; HRMS-EI (m/z): $\left[\mathrm{M}^{+}\right]$calcd for $\mathrm{C}_{12} \mathrm{H}_{15} \mathrm{~N}$ : 173.1204 found 173.1206 .<smiles>CCCc1cccc(Cl)c1C#N</smiles>

2-Butyl-6-chloro-benzonitrile (27): Following the general procedure above on $0.2 \mathrm{mmol}$ of 1-chloro-2-iodobenzene and iodobutane as the alkylating agent. The crude product was 
purified by column chromatography using $20 \%$ dichloromethane in hexane to give $23 \mathrm{mg}$ (59\% Yield) as a clear colorless oil. ${ }^{1} \mathrm{H} \mathrm{NMR}\left(\mathrm{CDCl}_{3}, 400 \mathrm{MHz}\right) \delta 7.42(\mathrm{t}, 1 \mathrm{H}, \mathrm{J}=7.9 \mathrm{~Hz})$, $7.33(\mathrm{~d}, 1 \mathrm{H}, \mathrm{J}=8.1 \mathrm{~Hz}), 7.21(\mathrm{~d}, 1 \mathrm{H}, \mathrm{J}=7.7 \mathrm{~Hz}), 2.89-2.81(\mathrm{~m}, 2 \mathrm{H}), 1.71-1.60(\mathrm{~m}, 2 \mathrm{H})$, 1.47-1.32 (m, 2H), $0.95(\mathrm{t}, 3 \mathrm{H}, \mathrm{J}=7.3 \mathrm{~Hz}) ;{ }^{13} \mathrm{C}-\mathrm{NMR}\left(\mathrm{CDCl}_{3}, 75 \mathrm{MHz}\right) \delta$ 149.6, 137.3, 133.3, 127.7, 127.3, 115.4, 113.6, 34.9, 33.0, 22.5, 14.0; IR (film) 2958, 2931, 2869,

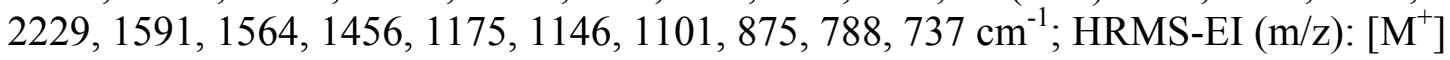
calcd for $\mathrm{C}_{11} \mathrm{H}_{12} \mathrm{NCl}$ : 193.0658 found 193.0656.

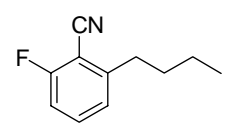

2-Butyl-6-fluoro-benzonitrile (28): Following the general procedure above on $0.2 \mathrm{mmol}$ of 1-fluoro-2-iodobenzene and iodobutane as the alkylating agent. The crude product was purified by column chromatography using $20 \%$ dichloromethane in hexane to give $11 \mathrm{mg}$ (31\% Yield) as a clear colorless oil. ${ }^{1} \mathrm{H}$ NMR $\left(\mathrm{CDCl}_{3}, 400 \mathrm{MHz}\right) \delta 7.48(\mathrm{~m}, 1 \mathrm{H}), 7.11(\mathrm{~d}$, $1 \mathrm{H}, \mathrm{J}=7.8 \mathrm{~Hz}), 7.02(\mathrm{dt}, 1 \mathrm{H}, \mathrm{J}=0.6 \mathrm{~Hz}, \mathrm{~J}=8.6 \mathrm{~Hz}), 2.88-2.80(\mathrm{~m}, 2 \mathrm{H}), 1.72-1.60(\mathrm{~m}, 2 \mathrm{H})$, 1.47-1.33 (m, 2H), 0.96 (t, 3H, J=7.3Hz); 19F-NMR $\left(\mathrm{CDCl}_{3}, 400 \mathrm{MHz}\right),-106.34$ (dd, $\mathrm{J}=5.8 \mathrm{~Hz}, \mathrm{~J}=8.7 \mathrm{~Hz}) ;{ }^{13} \mathrm{C}-\mathrm{NMR}\left(\mathrm{CDCl}_{3}, 100 \mathrm{MHz}\right) \delta 165.1,162.5,149.4,134.3(\mathrm{~d}, 1 \mathrm{H}$, $\mathrm{J}=8.9 \mathrm{~Hz}), 125.2(\mathrm{~d}, 1 \mathrm{H}, \mathrm{J}=3.3 \mathrm{~Hz}), 113.5(\mathrm{~d}, 1 \mathrm{H}, \mathrm{J}=19.6 \mathrm{~Hz}), 101.9(\mathrm{~d}, 1 \mathrm{H}, \mathrm{J}=14.6 \mathrm{~Hz})$, 34.4 (d, 1H, J=1.8Hz), 33.0 , 22.5 , 14.0; IR (film) 2959, 2871, 2230, 1614, 1574, 1471, 1268, 1104, $792 \mathrm{~cm}^{-1}$; HRMS-EI $(\mathrm{m} / \mathrm{z}):\left[\mathrm{M}^{+}\right]$calcd for $\mathrm{C}_{11} \mathrm{H}_{12} \mathrm{NF}: 177.0953$ found 177.0949.<smiles>CCCCc1cccc(OC)c1C#N</smiles>

2-Butyl-6-methoxy-benzonitrile (29): Following the general procedure above on 0.2 mmol of 1-iodo-2methoxybenzene and iodobutane as the alkylating agent. The crude product was purified by column chromatography using $40 \%$ dichloromethane in hexane to give $21 \mathrm{mg}\left(56 \%\right.$ Yield) as a clear colorless oil. ${ }^{1} \mathrm{H}$ NMR $\left(\mathrm{CDCl}_{3}, 400 \mathrm{MHz}\right) \delta 7.42(\mathrm{t}$, $1 \mathrm{H}, \mathrm{J}=8.1 \mathrm{~Hz}), 6.87(\mathrm{~d}, 1 \mathrm{H}, \mathrm{J}=7.7 \mathrm{~Hz}), 6.78(\mathrm{~d}, 1 \mathrm{H}, \mathrm{J}=8.4 \mathrm{~Hz}), 3.91(\mathrm{~s}, 3 \mathrm{H}), 2.85-2.75(\mathrm{~m}$, $2 \mathrm{H}), 1.70-1.58(\mathrm{~m}, 2 \mathrm{H}), 1.47-1.33(\mathrm{~m}, 2 \mathrm{H}), 0.94$ (t, 3H, J=7.3Hz); ${ }^{13} \mathrm{C}-\mathrm{NMR}\left(\mathrm{CDCl}_{3}, 100\right.$ MHz) $\delta 161.7,148.8,133.5,121.3,115.6,108.2,101.9,56.0,34.3,32.8,22.3,13.8 ;$ IR (film) 2957, 2932, 2865, 2221, 1580, 1475, 1282, 1111, 1080, 1055, 783, 741 $\mathrm{cm}^{-1}$; HRMS-EI (m/z): [ $\left.\mathrm{M}^{+}\right]$calcd for $\mathrm{C}_{12} \mathrm{H}_{15} \mathrm{NO}: 189.1153$ found 189.1156 .

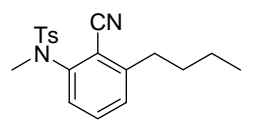

N-(3-Butyl-2-cyano-phenyl)-4,N-dimethyl-benzenesulfonamide (30): Following the general procedure above on $0.2 \mathrm{mmol}$ of $\mathrm{N}$-(2-iodophenyl)-4,N-

dimethylbenzenesulfonamide and iodobutane as the alkylating agent. The crude product was purified by column chromatography using 50\% dichloromethane in hexane to give $29 \mathrm{mg}\left(42 \%\right.$ Yield) as a clear viscous colorless oil. ${ }^{1} \mathrm{H} \mathrm{NMR}\left(\mathrm{CDCl}_{3}, 400 \mathrm{MHz}\right) \delta 7.67(\mathrm{~d}$, $2 \mathrm{H}, \mathrm{J}=8.3 \mathrm{~Hz}), 7.45(\mathrm{t}, 1 \mathrm{H}, \mathrm{J}=7.9 \mathrm{~Hz}), 7.31(\mathrm{~d}, 2 \mathrm{H}, \mathrm{J}=8.0 \mathrm{~Hz}), 7.27(\mathrm{~d}, 1 \mathrm{H}, \mathrm{J}=7.9 \mathrm{~Hz}), 7.08$ $(\mathrm{dd}, 1 \mathrm{H}, \mathrm{J}=1.0 \mathrm{~Hz}, \mathrm{~J}=8.0 \mathrm{~Hz}), 3.22(\mathrm{~s}, 3 \mathrm{H}), 2.86-2.78(\mathrm{~m}, 2 \mathrm{H}), 2.44(\mathrm{~s}, 3 \mathrm{H}), 1.68-1.56(\mathrm{~m}$, 2H), 1.44-1.32 (m, 2H), 0.94 (t, 3H, J=7.3Hz); ${ }^{13} \mathrm{C}-\mathrm{NMR}\left(\mathrm{CDCl}_{3}, 75 \mathrm{MHz}\right) \delta 148.9$, $144.2,134.3,132.7,129.7,129.4,128.9,128.3,126.5,115.4,114.0,38.6,34.5,32.9$, 22.3, 21.6, 13.8; IR (film) 2957, 2869, 2227, 1596, 1558, 1468, 1457, 1354, 1170, 1156, 
1089, 951, 815, 747, $668 \mathrm{~cm}^{-1}$; HRMS-ESI (m/z): $\left[\mathrm{M}^{+}\right]$calcd for $\mathrm{C}_{19} \mathrm{H}_{22} \mathrm{~N}_{2} \mathrm{O}_{2} \mathrm{~S}: 343.1474$ found 343.1469 .

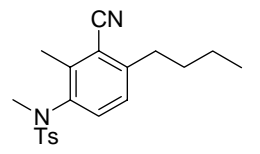

N-(4-Butyl-3-cyano-2-methyl-phenyl)-4,N-dimethyl-benzenesulfonamide (31):

Following the general procedure above on $0.2 \mathrm{mmol}$ of N-(3-iodo-2-methylphenyl)-4,Ndimethylbenzenesulfonamide and iodobutane as the alkylating agent. The crude product was purified by column chromatography using $50 \%$ dichloromethane in hexane to give $30 \mathrm{mg}(41 \%$ Yield $)$ as a clear viscous colorless oil. ${ }^{1} \mathrm{H} \mathrm{NMR}\left(\mathrm{CDCl}_{3}, 300 \mathrm{MHz}\right) \delta 7.61$ $7.55(\mathrm{~m}, 2 \mathrm{H}), 7.35-7.29(\mathrm{~m}, 2 \mathrm{H}), 7.01(\mathrm{~d}, 1 \mathrm{H}, \mathrm{J}=8.2 \mathrm{~Hz}), 6.78(\mathrm{~d}, 1 \mathrm{H}, \mathrm{J}=8.2 \mathrm{~Hz}), 3.10(\mathrm{~s}$, $3 \mathrm{H}), 2.85-2.76(\mathrm{~m}, 2 \mathrm{H}), 2.55(\mathrm{~s}, 3 \mathrm{H}), 2.46(\mathrm{~s}, 3 \mathrm{H}), 1.70-1.58(\mathrm{~m}, 2 \mathrm{H}), 1.43-1.35(\mathrm{~m}, 2 \mathrm{H})$, 0.95 (t, $1 \mathrm{H}, \mathrm{J}=7.3 \mathrm{~Hz}) ;{ }^{13} \mathrm{C}-\mathrm{NMR}\left(\mathrm{CDCl}_{3}, 75 \mathrm{MHz}\right) \delta 147.3,144.0,143.3,138.8,134.6$, 131.2, 129.7, 128.0, 127.3, 116.6, 115.0, 38.8, 34.5, 32.8, 22.4, 21.6, 17.3, 13.8; IR (film) 2955, 2870, 2221, 1734, 1653, 1597, 1558, 1480, 1349, 1185, 1155, 1089, 1033, 887, 810, 710, $667 \mathrm{~cm}^{-1}$; HRMS-EI (m/z): $\left[\mathrm{M}^{+}\right]$calcd for $\mathrm{C}_{20} \mathrm{H}_{24} \mathrm{~N}_{2} \mathrm{O}_{2} \mathrm{~S}: 356.1558$ found 356.1556 .<smiles>CCCCc1cc(Cl)cc(CCCC)c1O</smiles>

2,6-Dibutyl-4-chloro-benzonitrile (32): Following the general procedure above on 0.2 mmol of 1-chloro-4-iodobenzene and iodobutane as the alkylating agent. The crude product was purified by column chromatography using $20 \%$ dichloromethane in hexane to give $37 \mathrm{mg}\left(72 \%\right.$ Yield) as a clear colorless oil. ${ }^{1} \mathrm{H}$ NMR $\left(\mathrm{CDCl}_{3}, 300 \mathrm{MHz}\right) \delta 7.13$ (s, $2 \mathrm{H}), 2.84-2.76(\mathrm{~m}, 4 \mathrm{H}), 1.70-1.58(\mathrm{~m}, 4 \mathrm{H}), 1.48-1.34(\mathrm{~m}, 4 \mathrm{H}), 0.96(\mathrm{t}, 6 \mathrm{H}, \mathrm{J}=7.3 \mathrm{~Hz}),{ }^{13} \mathrm{C}-$ NMR $\left(\mathrm{CDCl}_{3}, 75 \mathrm{MHz}\right) \delta 147.8,137.5,125.9,115.5,109.9,33.4,31.7,21.3,12.7$; IR (film) 3065, 2949, 2928, 2874, 2218, 1582, 1456, 1416, 1378, 1263, 1174, 1106, 1018, 939, $865,729 \mathrm{~cm}^{-1}$; HRMS-EI (m/z): [ $\left.\mathrm{M}^{+}\right]$calcd for $\mathrm{C}_{15} \mathrm{H}_{20} \mathrm{NCl}: 249.1284$ found 249.1283.<smiles>CCCCc1ccc(CCCC)c(CCCC)c1</smiles>

2,6-Dibutyl-4-methoxy-benzonitrile (33): Following the general procedure above on 0.2 mmol of 1-iodo-4-methoxybenzene and iodobutane as the alkylating agent. The crude product was purified by column chromatography using $40 \%$ dichloromethane in hexane to give $35 \mathrm{mg}\left(71 \%\right.$ Yield) as a clear colorless oil. ${ }^{1} \mathrm{H}$ NMR $\left(\mathrm{CDCl}_{3}, 400 \mathrm{MHz}\right) \delta 6.63(\mathrm{~s}$, $2 \mathrm{H}), 3.83(\mathrm{~s}, 3 \mathrm{H}), 2.83-2.75(\mathrm{~m}, 4 \mathrm{H}), 1.71-1.60(\mathrm{~m}, 4 \mathrm{H}), 1.47-1.33(\mathrm{~m}, 4 \mathrm{H}), 0.95(\mathrm{t}, 6 \mathrm{H}$, $\mathrm{J}=7.3 \mathrm{~Hz}) ;{ }^{13} \mathrm{C}-\mathrm{NMR}\left(\mathrm{CDCl}_{3}, 100 \mathrm{MHz}\right) \delta 162.3,149.2,117.7,112.2,104.3,55.3,34.7$, 32.9, 22.4, 13.8; IR (film) 2962, 2922, 2853, 2212, 1599, 1575, 1467, 1443, 1337, 1297,

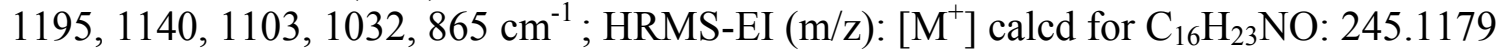
found 245.1780 . 


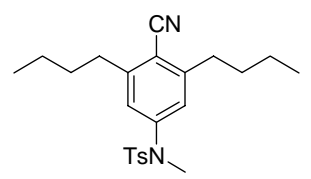

N-(3,5-Dibutyl-4-cyano-phenyl)-4,N-dimethyl-benzenesulfonamide (34): Following the general procedure above on $0.2 \mathrm{mmol}$ of $\mathrm{N}$-(4-iodophenyl)-4,N-

dimethylbenzenesulfonamide and iodobutane as the alkylating agent. The crude product was purified by column chromatography using 50\% dichloromethane in hexane to give $50 \mathrm{mg}\left(72 \%\right.$ Yield) as a clear viscous colorless oil. ${ }^{1} \mathrm{H}$ NMR $\left(\mathrm{CDCl}_{3}, 300 \mathrm{MHz}\right) \delta 7.40(\mathrm{~d}$, $2 \mathrm{H}, \mathrm{J}=8.3 \mathrm{~Hz}), 7.24(\mathrm{~d}, 2 \mathrm{H}, \mathrm{J}=8.0 \mathrm{~Hz}), 6.88(\mathrm{~s}, 2 \mathrm{H}), 3.16(\mathrm{~s}, 3 \mathrm{H}), 2.81-2.73(\mathrm{~m}, 4 \mathrm{H}), 2.42$ $(\mathrm{s}, 3 \mathrm{H}), 1.61-1.51(\mathrm{~m}, 4 \mathrm{H}), 1.42-1.28(\mathrm{~m}, 4 \mathrm{H}), 0.93(\mathrm{t}, 6 \mathrm{H}, \mathrm{J}=7.3 \mathrm{~Hz}) ;{ }^{13} \mathrm{C}-\mathrm{NMR}$ $\left(\mathrm{CDCl}_{3}, 75 \mathrm{MHz}\right) \delta 148.0,144.9,143.9,133.2,129.4,127.7,123.9,116.7,110.6,37.5$, 34.5, 32.8, 22.3, 21.5, 13.8; IR (film) 2957, 2871, 2218, 1599, 1465, 1355, 1306, 1186 , 1166, 1089, 960, 805, 784, $666 \mathrm{~cm}^{-1}$; HRMS-EI (m/z): $\left[\mathrm{M}^{+}\right]$calcd for $\mathrm{C}_{23} \mathrm{H}_{30} \mathrm{~N}_{2} \mathrm{O}_{2} \mathrm{~S}$ : 398.2028 found 398.2028 .

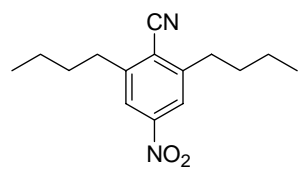

2,6-Dibutyl-4-nitro-benzonitrile (35): Following the general procedure above on 0.2 mmol of 1-iodo-4-nitrobenzene and iodobutane as the alkylating agent. The crude product was purified by column chromatography using 50\% dichloromethane in hexane to give $14 \mathrm{mg}\left(26 \%\right.$ Yield) as a yellow viscous oil. ${ }^{1} \mathrm{H} \mathrm{NMR}\left(\mathrm{CDCl}_{3}, 400 \mathrm{MHz}\right) \delta 7.99$ (s, 2H), $2.95(\mathrm{~m}, 4 \mathrm{H}), 1.70(\mathrm{~m}, 4 \mathrm{H}), 1.44(\mathrm{~m}, 4 \mathrm{H}), 0.98(\mathrm{t}, 6 \mathrm{H}, \mathrm{J}=7.3 \mathrm{~Hz}) ;{ }^{13} \mathrm{C}-\mathrm{NMR}\left(\mathrm{CDCl}_{3}\right.$, $100 \mathrm{MHz}) \delta 149.7,149.3,121.2,118.3,115.4,34.6,32.6,22.3,13.7$; IR (film) 3084, 2058, 2871, 2223, 1593, 1531, 1456, 1350, 1106, 901, 793, $746 \mathrm{~cm}^{-1}$; HRMS-EI (m/z): $\left[\mathrm{M}^{+}\right]$calcd for $\mathrm{C}_{15} \mathrm{H}_{20} \mathrm{~N}_{2} \mathrm{O}_{2}: 260.1524$ found 260.1527 .<smiles>CC(C)Cc1ccc2ccccc2c1C#N</smiles>

2-Isobutyl-naphthalene-1-carbonitrile (36): Following the general procedure above on $0.2 \mathrm{mmol}$ of iodonaphthalene and 1-iodo-2-methylpropane as the alkylating agent. The crude product was purified by column chromatography using $20 \%$ dichloromethane in hexane to give $32 \mathrm{mg}\left(62 \%\right.$ Yield) as a clear colorless oil. ${ }^{1} \mathrm{H} \mathrm{NMR}\left(\mathrm{CDCl}_{3}, 400 \mathrm{MHz}\right) \delta$ $8.23(\mathrm{dd}, 1 \mathrm{H}, \mathrm{J}=0.9 \mathrm{~Hz}, \mathrm{~J}=8.4 \mathrm{~Hz}), 7.97(\mathrm{~d}, 1 \mathrm{H}, \mathrm{J}=8.5 \mathrm{~Hz}), 7.88(\mathrm{~d}, 1 \mathrm{H}, \mathrm{J}=8.2 \mathrm{~Hz}), 7.67$ $(\mathrm{ddd}, 1 \mathrm{H}, \mathrm{J}=1.3 \mathrm{~Hz}, \mathrm{~J}=6.9 \mathrm{~Hz}, \mathrm{~J}=8.3 \mathrm{~Hz}), 7.56(\mathrm{ddd}, 1 \mathrm{H}, \mathrm{J}=1.2 \mathrm{~Hz}, \mathrm{~J}=6.9 \mathrm{~Hz}, \mathrm{~J}=8.1 \mathrm{~Hz}), 7.38$ $(\mathrm{d}, 1 \mathrm{H}, \mathrm{J}=8.5 \mathrm{~Hz}), 2.93(\mathrm{~d}, 2 \mathrm{H}, \mathrm{J}=7.3 \mathrm{~Hz}), 2.18-2.02(\mathrm{~m}, 1 \mathrm{H}), 1.00(\mathrm{~d}, 6 \mathrm{H}, \mathrm{J}=6.6 \mathrm{~Hz}) ;{ }^{13} \mathrm{C}-$ $\mathrm{NMR}\left(\mathrm{CDCl}_{3}, 75 \mathrm{MHz}\right) \delta 146.9,133.0,132.6,131.5,128.6,128.5,127.6,126.8,125.3$, 117.4, 109.6, 44.5, 30.7, 22.5; IR (film) 3058, 2957, 2927, 2868, 2214, 1623, 1594, 1507, 1466, 1385, 1367, 1270, 1220, 1166, 1097, 1027, 952, 868, 837, 809, 780, 752, 691, 663

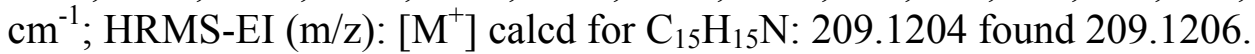

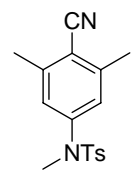


N-(4-Cyano-3,5-dimethyl-phenyl)-4,N-dimethyl-benzenesulfonamide (37): Following the general procedure above on $0.2 \mathrm{mmol}$ of and iodomethane as the alkylating agent.

The crude product was purified by column chromatography using $10 \%$ ether in hexane to give $23 \mathrm{mg}$ (37\% Yield) as a beige solid $(\mathrm{mp}=139-140)$. $1 \mathrm{H}-\mathrm{NMR}\left(\mathrm{CDCl}_{3}, 400 \mathrm{MHz}\right) \delta$ 7.47-7.41 (m, 2H), 7.30-7.24 (m, 2H), $6.92(\mathrm{~s}, 2 \mathrm{H}), 3.14(\mathrm{~s}, 3 \mathrm{H}), 2.48(\mathrm{~s}, 6 \mathrm{H}), 2.43(\mathrm{~s}$, $3 \mathrm{H}) ;{ }^{13} \mathrm{C}-\mathrm{NMR}\left(\mathrm{CDCl}_{3}, 100 \mathrm{MHz}\right) \delta 145.1,144.2,143.1,133.5,129.7,127.9,124.5$, 117.0, 111.7, 37.7, 21.7, 21.0; IR (film) 3013, 2956, 2398, 2223, 1599, 1353, 1214, 1166 $\mathrm{cm}^{-1}$; HRMS-EI (m/z): $\left[\mathrm{M}^{+}\right]$calcd for $\mathrm{C}_{17} \mathrm{H}_{18} \mathrm{~N}_{2} \mathrm{O}_{2} \mathrm{~S}: 314.1089$ found 314.1097.

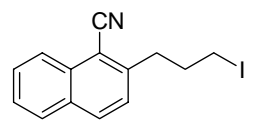

2-(3-Iodo-propyl)-naphthalene-1-carbonitrile (38): Following the general procedure above on $0.2 \mathrm{mmol}$ of iodonaphthalene and 1,3-diiodopropane as the alkylating agent. The crude product was purified by column chromatography using $20 \%$ dichloromethane in hexane to give $29 \mathrm{mg}$ ( $45 \%$ Yield) as a clear colorless oil. ${ }^{1} \mathrm{H} \mathrm{NMR}\left(\mathrm{CDCl}_{3}, 300 \mathrm{MHz}\right)$ $\delta 8.22(\mathrm{~d}, 1 \mathrm{H}, \mathrm{J}=8.7 \mathrm{~Hz}), 8.01(\mathrm{~d}, 1 \mathrm{H}, \mathrm{J}=8.5 \mathrm{~Hz}), 7.90(\mathrm{~d}, 1 \mathrm{H}, \mathrm{J}=8.2 \mathrm{~Hz}), 7.73-7.64(\mathrm{~m}, 1 \mathrm{H})$, 7.63-7.54 (m, 1H), $7.46(\mathrm{~d}, 1 \mathrm{H}, \mathrm{J}=8.5 \mathrm{~Hz}), 3.24(\mathrm{t}, 2 \mathrm{H}, \mathrm{J}=6.9 \mathrm{~Hz}), 3.21-3.13(\mathrm{~m}, 2 \mathrm{H}), 2.35-$ $2.23(\mathrm{~m}, 2 \mathrm{H}) ;{ }^{13} \mathrm{C}-\mathrm{NMR}\left(\mathrm{CDCl}_{3}, 75 \mathrm{MHz}\right) \delta 145.3,133.0,131.5,128.7,128.4,127.5$, 126.9, 126.7, 125.0, 116.7, 109.2, 35.9, 34.4, 5.0; IR (film) 3054, 2936, 2861, 2355, $2215,1683,1622,1593,1558,1506,1456,1429,1374,1218,1169,1025,825,751,668$ $\mathrm{cm}^{-1}$; HRMS-EI (m/z): [M+] calcd for $\mathrm{C}_{14} \mathrm{H}_{12} \mathrm{NI}$ : 321.0014 found 321.0018 .

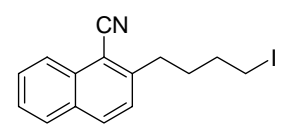

2-(4-Iodo-butyl)-naphthalene-1-carbonitrile (39): Following the general procedure above on $0.2 \mathrm{mmol}$ of iodonaphthalene and 1,4-diiodobutane as the alkylating agent. The crude product was purified by column chromatography using $20 \%$ dichloromethane in hexane to give $34 \mathrm{mg}$ (49\% Yield) as a clear colorless oil. ${ }^{1} \mathrm{H} \mathrm{NMR}\left(\mathrm{CDCl}_{3}, 300 \mathrm{MHz}\right) \delta$ $8.22(\mathrm{~d}, 1 \mathrm{H}, \mathrm{J}=8.3 \mathrm{~Hz}), 8.01(\mathrm{~d}, 1 \mathrm{H}, \mathrm{J}=8.5 \mathrm{~Hz}), 7.89$ (d, 1H, J=8.1Hz), 7.68 (ddd, $1 \mathrm{H}$, $\mathrm{J}=1.3 \mathrm{~Hz}, \mathrm{~J}=7.0 \mathrm{~Hz}, \mathrm{~J}=8.3 \mathrm{~Hz}), 7.57(\mathrm{ddd}, 1 \mathrm{H}, \mathrm{J}=1.1 \mathrm{~Hz}, \mathrm{~J}=7.0 \mathrm{~Hz}, \mathrm{~J}=8.1 \mathrm{~Hz}), 7.43(\mathrm{~d}, 1 \mathrm{H}$, $\mathrm{J}=8.5 \mathrm{~Hz}), 3.24(\mathrm{t}, 2 \mathrm{H}, \mathrm{J}=6.5 \mathrm{~Hz}), 3.08(\mathrm{t}, 2 \mathrm{H}, \mathrm{J}=7.3 \mathrm{~Hz}), 2.01-1.79(\mathrm{~m}, 4 \mathrm{H}) ;{ }^{13} \mathrm{C}-\mathrm{NMR}$ $\left(\mathrm{CDCl}_{3}, 75 \mathrm{MHz}\right) \delta 146.6,132.9,132.7,131.4,128.6,128.3,126.8,126.6,125.0,116.9$, 108.9, 34.1, 32.7, 31.8, 6.1; IR (film) 3054, 2953, 2357, 2216, 1700, 1653, 1592, 1558, 1506, 1425, 1375, 1340, 1269, 1207, 1170, 868, 823, 802, 773, 752, $668 \mathrm{~cm}^{-1}$; HRMS-EI $(\mathrm{m} / \mathrm{z}):\left[\mathrm{M}^{+}\right]$calcd for $\mathrm{C}_{15} \mathrm{H}_{14} \mathrm{NI}: 335.0171$ found 335.0170 .

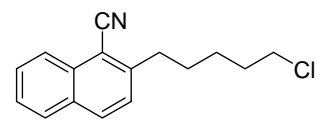

2-(5-Chloro-pentyl)-naphthalene-1-carbonitrile (40): Following the general procedure above on $0.2 \mathrm{mmol}$ of iodonaphthalene and 1-chloro-5-iodopentane as the alkylating agent. The crude product was purified by column chromatography using $20 \%$ dichloromethane in hexane to give $28 \mathrm{mg}$ (54\% Yield) as a clear colorless oil. ${ }^{1} \mathrm{H}$ NMR $\left(\mathrm{CDCl}_{3}, 400 \mathrm{MHz}\right) \delta 8.22(\mathrm{dd}, 1 \mathrm{H}, \mathrm{J}=0.9 \mathrm{~Hz}, \mathrm{~J}=8.4 \mathrm{~Hz}), 7.99(\mathrm{~d}, 1 \mathrm{H}, \mathrm{J}=8.5 \mathrm{~Hz}), 7.88(\mathrm{~d}$, $1 \mathrm{H}, \mathrm{J}=8.2 \mathrm{~Hz}$ ), 7.67 (ddd, $1 \mathrm{H}, \mathrm{J}=1.3 \mathrm{~Hz}, \mathrm{~J}=6.9 \mathrm{~Hz}, \mathrm{~J}=8.3 \mathrm{~Hz}$ ), 7.56 (ddd, $1 \mathrm{H}, \mathrm{J}=1.2 \mathrm{~Hz}$, $\mathrm{J}=6.9 \mathrm{~Hz}, \mathrm{~J}=8.1 \mathrm{~Hz}), 7.41(\mathrm{~d}, 1 \mathrm{H}, \mathrm{J}=8.5 \mathrm{~Hz}), 3.55(\mathrm{t}, 2 \mathrm{H}, \mathrm{J}=6.6 \mathrm{~Hz}), 3.10-3.03(\mathrm{~m}, 2 \mathrm{H})$, 
1.91-1.74 (m, 4H), 1.63-1.54 (m, 2H); ${ }^{13} \mathrm{C}-\mathrm{NMR}\left(\mathrm{CDCl}_{3}, 100 \mathrm{MHz}\right) \delta 147.4,133.0,131.6$, $128.8,128.5,127.0,126.9,125.2,117.1,109.1,104.9,45.0,35.4,32.5,30.5,26.7$; IR (film) 2937, 2861, 2215, 1593, 1506, 1457, 1375, 1146, 1026, 824, 752, 668, $646 \mathrm{~cm}^{-1}$; HRMS-EI (m/z): [ $\left.\mathrm{M}^{+}\right]$calcd for $\mathrm{C}_{16} \mathrm{H}_{16} \mathrm{NCl}$ : 257.0971 found 257.0964.

\section{General Procedure for Intermolecular Alkylation/Cyanation Reaction} Using Alkyl Chlorides: To a $5 \mathrm{ml}$ microwave vial containing a magnetic stir bar was added 1.0 equivalent aryl iodide, 1.5 equivalents alkyl chloride, 0.1 equivalents palladium acetate, 0.22 equivalents $\mathrm{Bu}_{3} \mathrm{PHBF}_{4}, 2.0$ equivalents $\mathrm{K}_{4}\left[\mathrm{Fe}(\mathrm{CN})_{6}\right] 3 \mathrm{H}_{2} \mathrm{O}, 6.0$ equivalents norbornene and 3.0 equivalents cesium carbonate. The vial was then sealed and flushed with argon. Acetonitrile was added to give a final concentration of $0.05 \mathrm{M}$ based upon aryl iodide. The resulting solution was then purged with argon for 5 minutes followed by heating with stirring in an oil bath preheated to $90{ }^{\circ} \mathrm{C}$ for 24 hours. The mixture was filtered through celite with dichloromethane, dried with sodium sulphate and evaporated under reduced pressure. The product was then purified by flash column chromatography.

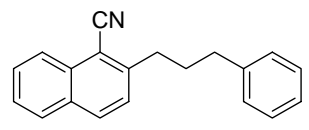

2-(3-Phenyl-propyl)-naphthalene-1-carbonitrile (41): Following the general procedure above on $0.2 \mathrm{mmol}$ of iodonaphthalene and 1-chloro-3-phenylpropane as the alkyl chloride. The crude product was purified by column chromatography using $20 \%$ dichloromethane in hexane to give $43 \mathrm{mg}$ ( $79 \%$ Yield) as a clear colorless oil. ${ }^{1} \mathrm{H}$ NMR $\left(\mathrm{CDCl}_{3}, 400 \mathrm{MHz}\right) \delta 8.22(\mathrm{dd}, 1 \mathrm{H}, \mathrm{J}=0.9 \mathrm{~Hz}, \mathrm{~J}=8.4 \mathrm{~Hz}), 7.97(\mathrm{~d}, 1 \mathrm{H}, \mathrm{J}=8.5 \mathrm{~Hz}), 7.87(\mathrm{~d}$, $1 \mathrm{H}, \mathrm{J}=8.2 \mathrm{~Hz}$ ), 7.67 (ddd, $1 \mathrm{H}, \mathrm{J}=1.3 \mathrm{~Hz}, \mathrm{~J}=6.9 \mathrm{~Hz}, \mathrm{~J}=8.3 \mathrm{~Hz}), 7.56(\mathrm{ddd}, 1 \mathrm{H}, \mathrm{J}=1.2 \mathrm{~Hz}$, $\mathrm{J}=6.9 \mathrm{~Hz}, \mathrm{~J}=8.1 \mathrm{~Hz}), 7.39(\mathrm{~d}, 1 \mathrm{H}, \mathrm{J}=8.5 \mathrm{~Hz}), 7.29(\mathrm{~m}, 2 \mathrm{H}), 7.19(\mathrm{~m}, 3 \mathrm{H}), 3.14-3.06(\mathrm{~m}$, 2H), 2.79-2.72 (m, 2H), 2.15-2.04 (m, 2H); ${ }^{13} \mathrm{C}-\mathrm{NMR}\left(\mathrm{CDCl}_{3}, 100 \mathrm{MHz}\right)$ 8147.4, 141.7, 133.0, 133.0, 131.6, 128.7, 128.6, 128.6, 128.5, 126.9, 126.9, 126.2, 125.2, 117.1, 109.2, 35.7, 35.2, 32.8; IR (film) 3059, 3025, 2944, 2861, 2215, 1943, 1733, 1593, 1507, 1496, 1453, 1375, 1270, 1214, 1147, 1029, 824, 748, 699, $668 \mathrm{~cm}^{-1}$; HRMS-EI (m/z): [M+] calcd for $\mathrm{C}_{20} \mathrm{H}_{17} \mathrm{~N}$ : 271.1361 found 271.1355.<smiles>CCCCC1=CC2C=CC=C2C1C</smiles>

2-Butyl-naphthalene-1-carbonitrile (42): Following the general procedure above on 0.2 mmol of iodonaphthalene and 1-chlorobutane as the alkyl chloride. The crude product was purified by column chromatography using $20 \%$ dichloromethane in hexane to give $10 \mathrm{mg}(23 \%$ Yield) as a clear colorless oil.

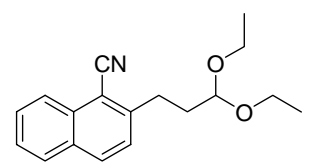

2-(3,3-Diethoxy-propyl)-naphthalene-1-carbonitrile (43): Following the general procedure above on $0.2 \mathrm{mmol}$ of iodonaphthalene and 3-chloro-1,1-diethoxypropane as the alkyl chloride. The crude product was purified by column chromatography using $20 \%$ dichloromethane in hexane to give $26 \mathrm{mg}$ (46\% Yield) as a clear colorless oil. ${ }^{1} \mathrm{H}$ NMR 
$\left(\mathrm{CDCl}_{3}, 400 \mathrm{MHz}\right) \delta 8.22(\mathrm{dd}, 1 \mathrm{H}, \mathrm{J}=0.6 \mathrm{~Hz}, \mathrm{~J}=8.3 \mathrm{~Hz}), 7.98(\mathrm{~d}, 1 \mathrm{H}, \mathrm{J}=8.5 \mathrm{~Hz}), 7.87(\mathrm{~d}$, $1 \mathrm{H}, \mathrm{J}=8.1 \mathrm{~Hz}$ ), 7.67 (ddd, $1 \mathrm{H}, \mathrm{J}=1.2 \mathrm{~Hz}, \mathrm{~J}=7.0 \mathrm{~Hz}, \mathrm{~J}=8.3 \mathrm{~Hz}$ ), 7.56 (ddd, $1 \mathrm{H}, \mathrm{J}=1.1 \mathrm{~Hz}$, $\mathrm{J}=7.0 \mathrm{~Hz}, \mathrm{~J}=8.0 \mathrm{~Hz}), 7.44(\mathrm{~d}, 1 \mathrm{H}, \mathrm{J}=8.5 \mathrm{~Hz}), 4.56(\mathrm{t}, 1 \mathrm{H}, \mathrm{J}=5.7 \mathrm{~Hz}), 3.69(\mathrm{qd}, 2 \mathrm{H}, \mathrm{J}=7.0 \mathrm{~Hz}$, $\mathrm{J}=9.3 \mathrm{~Hz}), 3.53(\mathrm{qd}, 2 \mathrm{H}, \mathrm{J}=7.1 \mathrm{~Hz}, \mathrm{~J}=9.3 \mathrm{~Hz}), 3.16-3.09(\mathrm{~m}, 2 \mathrm{H}), 2.12-2.03(\mathrm{~m}, 2 \mathrm{H}), 1.22$ $(\mathrm{t}, 6 \mathrm{H}, \mathrm{J}=7.1 \mathrm{~Hz}) ;{ }^{13} \mathrm{C}-\mathrm{NMR}\left(\mathrm{CDCl}_{3}, 100 \mathrm{MHz}\right) \delta 146.8,132.8,132.8,131.4,128.5,128.3$, 126.8, 126.7, 125.0, 116.8, 109.0, 101.9, 61.3, 34.6, 30.6, 15.3; IR (film) 3059, 2973, 2928, 2872, 2216, 1652, 1625, 1594, 1508, 1456, 1373, 1342, 1127, 1065, 827, 752, 667 $\mathrm{cm}^{-1}$; HRMS-EI $(\mathrm{m} / \mathrm{z}):\left[\mathrm{M}^{+}\right]$calcd for $\mathrm{C}_{18} \mathrm{H}_{21} \mathrm{NO}_{2}: 283.1572$ found 283.1568 .<smiles>C[AsH3]OCCc1ccc2ccccc2c1C#N</smiles>

2-[2-(tert-butyl-dimethyl-silanyloxy)-pentyl]-naphtalene-1-carbonitrile (44):

Following the general procedure above on $0.2 \mathrm{mmol}$ of iodonaphthalene and tert-butyl(2-chloropentoxy)-dimethylsilane as the alkyl chloride. The crude product was purified by column chromatography using $10 \%$ dichloromethane in hexane to give $49 \mathrm{mg}(67 \%$ Yield) as a clear colorless oil. ${ }^{1} \mathrm{H} \mathrm{NMR}\left(\mathrm{CDCl}_{3}, 400 \mathrm{MHz}\right) \delta 8.22(\mathrm{~d}, 1 \mathrm{H}, \mathrm{J}=8.4 \mathrm{~Hz}), 7.97$ $(\mathrm{d}, 1 \mathrm{H}, \mathrm{J}=8.6 \mathrm{~Hz}), 7.87(\mathrm{dd}, 1 \mathrm{H}, \mathrm{J}=0.5 \mathrm{~Hz}, \mathrm{~J}=8.1 \mathrm{~Hz}), 7.67(\mathrm{ddd}, 1 \mathrm{H}, \mathrm{J}=1.3 \mathrm{~Hz}, \mathrm{~J}=6.9 \mathrm{~Hz}$, $\mathrm{J}=8.3 \mathrm{~Hz}), 7.55(\mathrm{ddd}, 1 \mathrm{H}, \mathrm{J}=1.1 \mathrm{~Hz}, \mathrm{~J}=7.0 \mathrm{~Hz}, \mathrm{~J}=8.1 \mathrm{~Hz}), 7.41(\mathrm{~d}, 1 \mathrm{H}, \mathrm{J}=8.5 \mathrm{~Hz}), 3.60(\mathrm{t}$, $2 \mathrm{H}, \mathrm{J}=6.5 \mathrm{~Hz}), 3.08-3.01(\mathrm{~m}, 2 \mathrm{H}), 1.82-1.71(\mathrm{~m}, 2 \mathrm{H}), 1.55-1.48(\mathrm{~m}, 2 \mathrm{H}), 1.47-1.32(\mathrm{~m}$, $4 \mathrm{H}), 0.88(\mathrm{~s}, 9 \mathrm{H}), 0.03(\mathrm{~s}, 6 \mathrm{H}) ;{ }^{13} \mathrm{C}-\mathrm{NMR}\left(\mathrm{CDCl}_{3}, \mathrm{MHz}\right) \delta 147.7,132.8,132.6,131.3$, $128.3,126.8,125.0,117.0,108.8,63.1,35.3,32.7,31.1,29.0,25.9,25.6,18.3,-5.2$; IR (film) 2927, 2856, 2215, 1652, 1634, 1594, 1507, 1471, 1462, 1387, 1360, 1255, 1098, $1005,835,775,751,668 \mathrm{~cm}^{-1}$; HRMS-EI $(\mathrm{m} / \mathrm{z}):\left[\mathrm{M}^{+}\right]$calcd for $\mathrm{C}_{23} \mathrm{H}_{32} \mathrm{NOSi}: 366.2253$ found 366.2247 .

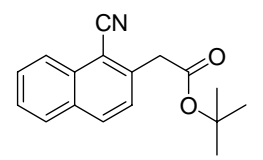

(1-Cyano-naphthalen-2-yl)-acetic acid tert-butyl ester (45): Following the general procedure above on $0.2 \mathrm{mmol}$ of iodonaphthalene and chloro-acetic acid- tert-butyl ester as the alkyl chloride. The crude product was purified by column chromatography using $30 \%$ dichloromethane in hexane to give $24 \mathrm{mg}$ ( $45 \%$ Yield) as a pale yellow crystalline solid(mp=83-84). Utilizing 3.0 eq of alkyl chloride: $33 \mathrm{mg}$ (62\% Yield). ${ }^{1} \mathrm{H}$ NMR $\left(\mathrm{CDCl}_{3}, 400 \mathrm{MHz}\right) \delta 8.24(\mathrm{dd}, 1 \mathrm{H}, \mathrm{J}=0.9 \mathrm{~Hz}, \mathrm{~J}=8.4 \mathrm{~Hz}), 8.03(\mathrm{~d}, 1 \mathrm{H}, \mathrm{J}=8.5 \mathrm{~Hz}), 7.90(\mathrm{~d}$, $1 \mathrm{H}, \mathrm{J}=8.2 \mathrm{~Hz}$ ), 7.69 (ddd, $1 \mathrm{H}, \mathrm{J}=1.3 \mathrm{~Hz}, \mathrm{~J}=6.9 \mathrm{~Hz}, \mathrm{~J}=8.3 \mathrm{~Hz}), 7.59$ (ddd, $1 \mathrm{H}, \mathrm{J}=1.2 \mathrm{~Hz}$, $\mathrm{J}=6.9 \mathrm{~Hz}, \mathrm{~J}=8.1 \mathrm{~Hz}), 7.50(\mathrm{~d}, 1 \mathrm{H}, \mathrm{J}=8.5 \mathrm{~Hz}), 4.00(\mathrm{~s}, 2 \mathrm{H}), 1.46(\mathrm{~s}, 9 \mathrm{H}) ;{ }^{13} \mathrm{C}-\mathrm{NMR}$ $\left(\mathrm{CDCl}_{3}, 100 \mathrm{MHz}\right) \delta 168.9,139.1,132.8,132.7,131.8,128.7,128.4,127.2,127.2,125.3$, 116.6, 110.5, 82.0, 41.7, 28.0; IR (film) 2955, 2927, 2871, 2217, 1734, 1700, 1594, 1457, 1368, 1329, 1273, 1253, 1213, 1149, 859, 846, 829, 773, 758, $677 \mathrm{~cm}^{-1}$; HRMS-EI $(\mathrm{m} / \mathrm{z}):\left[\mathrm{M}^{+}\right]$calcd for $\mathrm{C}_{17} \mathrm{H}_{17} \mathrm{NO}_{2}: 267.1259$ found 267.1259 .

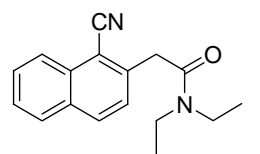

2-(1-Cyano-naphthalen-2-yl)-N,N-diethyl-acetamide (46): Following the general procedure above on $0.2 \mathrm{mmol}$ of iodonaphthalene and 3.0eq. of 2-chloro-N,N- 
diethylacetamide as the alkyl chloride. The crude product was purified by column chromatography using $50 \%$ dichloromethane in hexane to give $33 \mathrm{mg}(61 \%$ Yield) as a white crystalline solid(mp=123-124). ${ }^{1} \mathrm{H}$ NMR $\left(\mathrm{CDCl}_{3}, 400 \mathrm{MHz}\right) \delta 8.22(\mathrm{dd}, 1 \mathrm{H}$, $\mathrm{J}=0.9 \mathrm{~Hz}, \mathrm{~J}=8.4 \mathrm{~Hz}), 8.03(\mathrm{~d}, 1 \mathrm{H}, \mathrm{J}=8.6 \mathrm{~Hz}), 7.90(\mathrm{~d}, 1 \mathrm{H}, \mathrm{J}=8.2 \mathrm{~Hz}), 7.68$ (ddd, $1 \mathrm{H}$, $\mathrm{J}=1.3 \mathrm{~Hz}, \mathrm{~J}=6.9 \mathrm{~Hz}, \mathrm{~J}=8.3 \mathrm{~Hz}), 7.62-7.55(\mathrm{~m}, 2 \mathrm{H}), 4.13(\mathrm{~s}, 2 \mathrm{H}), 3.49-3.39(\mathrm{~m}, 4 \mathrm{H}), 1.25(\mathrm{t}$, $3 \mathrm{H}, \mathrm{J}=7.2 \mathrm{~Hz}), 1.15(\mathrm{t}, 3 \mathrm{H}, \mathrm{J}=7.1 \mathrm{~Hz}){ }^{13} \mathrm{C}-\mathrm{NMR}\left(\mathrm{CDCl}_{3}, 100 \mathrm{MHz}\right) \delta 168.2,140.7,132.9$, 132.8, 131.9, 128.8, 128.6, 127.5, 127.3, 125.4, 117.1, 110.1, 42.7, 40.8, 39.5, 14.5, 13.2; IR (chloroform solution) 2996, 2937, 2217, 1649, 1639, 1632, 1626, 1621, 1482, 1450, 1461, 1439, 1434, 1383, 1317, 1261, 1137, $1097 \mathrm{~cm}^{-1}$; HRMS-EI (m/z): [ $\left.\mathrm{M}^{+}\right]$calcd for $\mathrm{C}_{17} \mathrm{H}_{18} \mathrm{~N}_{2} \mathrm{O}: 266.1419$ found 266.1418 .

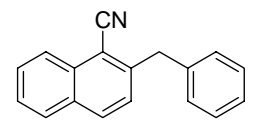

2-Benzyl-naphthalene-1-carbonitrile (47): Following the general procedure above on $0.2 \mathrm{mmol}$ of iodonaphthalene and chloromethylbenzene as the alkyl chloride. The crude product was purified by column chromatography using $20 \%$ dichloromethane in hexane to give $41 \mathrm{mg}\left(85 \%\right.$ Yield) as a clear viscous colorless oil. ${ }^{1} \mathrm{H} \mathrm{NMR}\left(\mathrm{CDCl}_{3}, 400 \mathrm{MHz}\right) \delta$ 8.25 (dd, $1 \mathrm{H}, \mathrm{J}=0.8 \mathrm{~Hz}, \mathrm{~J}=8.4 \mathrm{~Hz}), 7.94(\mathrm{~d}, 1 \mathrm{H}, \mathrm{J}=8.5 \mathrm{~Hz}), 7.85$ (d, $1 \mathrm{H}, \mathrm{J}=8.2 \mathrm{~Hz}), 7.68$ (ddd, $1 \mathrm{H}, \mathrm{J}=1.3 \mathrm{~Hz}, \mathrm{~J}=6.9 \mathrm{~Hz}, \mathrm{~J}=8.3 \mathrm{~Hz}$ ), 7.56 (ddd, $1 \mathrm{H}, \mathrm{J}=1.1 \mathrm{~Hz}, \mathrm{~J}=6.9 \mathrm{~Hz}, \mathrm{~J}=8.1 \mathrm{~Hz}$ ), 7.37 $(\mathrm{d}, 1 \mathrm{H}, \mathrm{J}=8.6 \mathrm{~Hz}), 7.33-7.20(\mathrm{~m}, 5 \mathrm{H}), 4.41(\mathrm{~s}, 2 \mathrm{H}) ;{ }^{13} \mathrm{C}-\mathrm{NMR}\left(\mathrm{CDCl}_{3}, 100 \mathrm{MHz}\right) \delta 145.6$, 138.9, 132.9, 132.7, 131.4, 128.9, 128.7, 128.6, 128.3, 127.0, 126.9, 126.7, 125.2, 117.1, 109.2, 40.9; IR (film) 3058, 3027, 2954, 2868, 2214, 1622, 1594, 1507, 1495, 1453, 1377, 1270, 1146, 1075, 839, 808, 780, 746, 722, 670, $667 \mathrm{~cm}^{-1}$; HRMS-EI (m/z): $\left[\mathrm{M}^{+}\right]$ calcd for $\mathrm{C}_{18} \mathrm{H}_{13} \mathrm{~N}$ : 243.1048 found 243.1052.

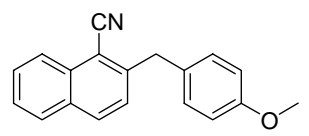

2-(4-Methoxy-benzyl)-naphthalene-1-carbonitrile (48): Following the general procedure above on $0.2 \mathrm{mmol}$ of iodonaphthalene and 1-chloromethyl-4-methoxybenzene as the alkyl chloride. The crude product was purified by column chromatography using $30 \%$ dichloromethane in hexane to give $18 \mathrm{mg}$ ( $33 \%$ Yield) as a white crystalline solid (mp=92-94). Utilizing 3.0 eq of alkyl chloride: $26 \mathrm{mg}$ ( $48 \%$ Yield). ${ }^{1} \mathrm{H} \mathrm{NMR}\left(\mathrm{CDCl}_{3}, 400\right.$ MHz) $\delta 8.24$ (dd, $1 \mathrm{H}, \mathrm{J}=0.5 \mathrm{~Hz}, \mathrm{~J}=8.4 \mathrm{~Hz}), 7.94(\mathrm{~d}, 1 \mathrm{H}, \mathrm{J}=8.6 \mathrm{~Hz}), 7.85(\mathrm{~d}, 1 \mathrm{H}, \mathrm{J}=8.1 \mathrm{~Hz})$, 7.67 (ddd, $1 \mathrm{H}, \mathrm{J}=1.2 \mathrm{~Hz}, \mathrm{~J}=7.0 \mathrm{~Hz}, \mathrm{~J}=8.3 \mathrm{~Hz}), 7.56(\mathrm{ddd}, 1 \mathrm{H}, \mathrm{J}=1.1 \mathrm{~Hz}, \mathrm{~J}=7.0 \mathrm{~Hz}, \mathrm{~J}=8.0 \mathrm{~Hz}$ ), $7.36(\mathrm{~d}, 1 \mathrm{H}, \mathrm{J}=8.6 \mathrm{~Hz}), 7.24-7.16(\mathrm{~m}, 2 \mathrm{H}), 6.88-6.80(\mathrm{~m}, 2 \mathrm{H}), 4.35(\mathrm{~s}, 2 \mathrm{H}), 3.77(\mathrm{~s}, 3 \mathrm{H})$; ${ }^{13} \mathrm{C}-\mathrm{NMR}\left(\mathrm{CDCl}_{3}, 100 \mathrm{MHz}\right) \delta 158.4,146.1,132.9,132.7,131.4,131.0,129.9,128.6$, 128.3, 127.0, 126.9, 125.2, 117.1, 114.1, 109.0, 55.2, 40.1; IR (chloroform solution) 2956, 2838, 2216, 1721, 1711, 1610, 1513, 1511, 1462, 1441, 1302, 1250, 1177, 1108, 1034, $820 \mathrm{~cm}^{-1}$; HRMS-EI (m/z): [ $\left.\mathrm{M}^{+}\right]$calcd for $\mathrm{C}_{19} \mathrm{H}_{15} \mathrm{NO}: 273.1153$ found 273.1156 .

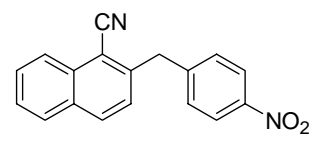

2-(4-Nitro-benzyl)-naphthalene-1-carbonitrile (49): Following the general procedure above on $0.2 \mathrm{mmol}$ of iodonaphthalene and 1-chloromethyl-4-nitrobenzene as the alkyl chloride. The crude product was purified by column chromatography using $30 \%$ 
dichloromethane in hexane to give $27 \mathrm{mg}$ ( $47 \%$ Yield) as a yellow crystalline solid $(\mathrm{mp}=114) .{ }^{1} \mathrm{H} \mathrm{NMR}\left(\mathrm{CDCl}_{3}, 300 \mathrm{MHz}\right) \delta 8.26(\mathrm{~d}, 1 \mathrm{H}, \mathrm{J}=8.3 \mathrm{~Hz}), 8.17(\mathrm{~d}, 1 \mathrm{H}, \mathrm{J}=8.5 \mathrm{~Hz})$, $8.02(\mathrm{~d}, 1 \mathrm{H}, \mathrm{J}=8.6 \mathrm{~Hz}), 7.90(\mathrm{~d}, 1 \mathrm{H}, \mathrm{J}=7.9 \mathrm{~Hz}), 7.72(\mathrm{t}, 1 \mathrm{H}, \mathrm{J}=7.6 \mathrm{~Hz}), 7.62(\mathrm{t}, 1 \mathrm{H}$, $\mathrm{J}=7.5 \mathrm{~Hz}), 7.45(\mathrm{~d}, 1 \mathrm{H}, \mathrm{J}=8.4 \mathrm{~Hz}), 7.37(\mathrm{~d}, 1 \mathrm{H}, \mathrm{J}=8.5 \mathrm{~Hz}), 4.52(\mathrm{~s}, 2 \mathrm{H}) ;{ }^{13} \mathrm{C}-\mathrm{NMR}\left(\mathrm{CDCl}_{3}\right.$, $100 \mathrm{MHz}) \delta 146.5,143.6,133.6,133.0,131.9,129.9,129.3,128.7,127.6,126.9,125.4$, 124.2, 124.0, 117.0, 110.0, 40.8; IR (film) 3062, 2946, 858, 2218, 1599, 1523, 1518, 1493, 1347, $11101016,857 \mathrm{~cm}^{-1}$; HRMS-EI $(\mathrm{m} / \mathrm{z})$ : $\left[\mathrm{M}^{+}\right]$calcd for $\mathrm{C}_{18} \mathrm{H}_{12} \mathrm{~N}_{2} \mathrm{O}_{2}$ : 288.0898 found 288.0895 .<smiles>Cc1cccc(CCCc2ccccc2)c1C#N</smiles>

2-Methyl-6-(3-phenyl-propyl)-benzonitrile (50): Following the general procedure above on $0.2 \mathrm{mmol}$ of 1-iodo-2-methylbenzene and 1-chloro-3-phenylproane as the alkyl chloride. The crude product was purified by column chromatography using $20 \%$ dichloromethane in hexane to give $39 \mathrm{mg}$ (83\% Yield) as a clear colorless oil. ${ }^{1} \mathrm{H}$ NMR $\left(\mathrm{CDCl}_{3}, 400 \mathrm{MHz}\right) \delta$ 7.39-7.33 (m, 1H), 7.32-7.26 (m, 2H), 7.23-7.16 (m, 3H), $7.13(\mathrm{~d}$, $1 \mathrm{H}, \mathrm{J}=7.7 \mathrm{~Hz}), 7.11(\mathrm{~d}, 1 \mathrm{H}, \mathrm{J}=7.7 \mathrm{~Hz}), 2.91-2.84(\mathrm{~m}, 2 \mathrm{H}), 2.74-2.67(\mathrm{~m}, 2 \mathrm{H}), 2.54(\mathrm{~s}, 3 \mathrm{H})$, 2.05-1.95(m, 2H) ${ }^{13} \mathrm{C}-\mathrm{NMR}\left(\mathrm{CDCl}_{3}, 100 \mathrm{MHz}\right) \delta 146.7,142.5,141.9,132.3,132.3$, 128.5, 127.7, 126.7, 126.1, 117.3, 113.1, 35.7, 34.7, 32.6, 21.0; IR(film) 3061, 3025, 2928, 2861, 2217, 1594, 1496, 1468, 1453, 1383, 1169, 1030, 783, 744, $699 \mathrm{~cm}^{-1}$; HRMS-EI $(\mathrm{m} / \mathrm{z}):\left[\mathrm{M}^{+}\right]$calcd for $\mathrm{C}_{17} \mathrm{H}_{17} \mathrm{~N}$ : 235.1361 found 235.1362 .<smiles>Clc1cccc(CCCc2ccccc2)c1Cl</smiles>

2-Chloro-6-(3-phenyl-propyl)-benzonitrile (51): Following the general procedure above on $0.2 \mathrm{mmol}$ of 1-chloro-2-iodobenzene and 1-chloro-3-phenylproane as the alkyl chloride. The crude product was purified by column chromatography using $20 \%$ dichloromethane in hexane to give $22 \mathrm{mg}\left(43 \%\right.$ Yield) as a clear colorless oil. ${ }^{1} \mathrm{H}$ NMR $\left(\mathrm{CDCl}_{3}, 400 \mathrm{MHz}\right) \delta$ 7.45-7.39 (m, 1H), 7.36-7.27 (m, 3H), 7.23-7.16 (m, 4H), 2.94-2.86 (m, 2H), 2.75-2.67 (m, 2H), 2.07-1.96(m, 2H); ${ }^{13} \mathrm{C}-\mathrm{NMR}\left(\mathrm{CDCl}_{3}, 100 \mathrm{MHz}\right) \delta 148.9$, 141.4, 137.4, 133.3, 128.6, 128.5, 127.6, 127.5, 126.2, 115.3, 113.6, 35.6, 34.8, 32.3; IR (film) 3061, 3026, 2926, 2860, 2229, 1652, 1591, 1564, 1496, 1455, 1440, 1204, 1177, $1133,1082,1030,890,790,750,700,668 \mathrm{~cm}^{-1}$; HRMS-EI $(\mathrm{m} / \mathrm{z}):\left[\mathrm{M}^{+}\right]$calcd for $\mathrm{C}_{16} \mathrm{H}_{14} \mathrm{NCl}: 254.0736$ found 254.0733.

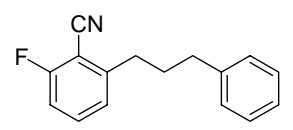

2-Fluoro-6-(3-phenyl-propyl)-benzonitrile (52): Following the general procedure above on $0.2 \mathrm{mmol}$ of 1-fluoro-2-iodobenzene and 1-chloro-3-phenylpropane as the alkyl chloride. The crude product was purified by column chromatography using $20 \%$ dichloromethane in hexane to give $19 \mathrm{mg}\left(40 \%\right.$ Yield) as a clear colorless oil. ${ }^{1} \mathrm{H}$ NMR $\left(\mathrm{CDCl}_{3}, 400 \mathrm{MHz}\right) \delta$ 7.52-7.44(m, 1H), 7.33-7.27 (m, 2H), 7.24-7.14 (m, 3H), $7.09(\mathrm{~d}$, $1 \mathrm{H}, \mathrm{J}=7.7 \mathrm{~Hz}), 7.03(\mathrm{ddd}, 1 \mathrm{H}, \mathrm{J}=1.3 \mathrm{~Hz}, \mathrm{~J}=6.9 \mathrm{~Hz}, \mathrm{~J}=8.3 \mathrm{~Hz}), 2.91-2.84(\mathrm{~m}, 2 \mathrm{H}), 2.74-2.68$ $(\mathrm{m}, 2 \mathrm{H}), 2.07-1.97(\mathrm{~m}, 2 \mathrm{H}) ; 19 \mathrm{~F}-\mathrm{NMR}\left(\mathrm{CDCl}_{3}, 400 \mathrm{MHz}\right) \delta-106.5(\mathrm{dd}, 1 \mathrm{H}, \mathrm{J}=5.9 \mathrm{~Hz}$, $\mathrm{J}=8.7 \mathrm{~Hz}) ;{ }^{13} \mathrm{C}-\mathrm{NMR}\left(\mathrm{CDCl}_{3}, 100 \mathrm{MHz}\right) \delta 163.8(\mathrm{~d}, \mathrm{~J}=258.5 \mathrm{~Hz}), 148.8,141.5,134.4(\mathrm{~d}$, $\mathrm{J}=8.9 \mathrm{~Hz}), 128.7128 .6,126.3,125.1(\mathrm{~d}, 1 \mathrm{H}, \mathrm{J}=3.3 \mathrm{~Hz}), 113.8$ (d, 1H, J=19.6Hz), 113.4 (d, 
$1 \mathrm{H}, \mathrm{J}=0.9 \mathrm{~Hz}$ ), 102.0 (d, 1H, J=14.8Hz), 35.634 .3 (d, 1H, J=1.9Hz), 32.4; IR (film) 3026, 2926, 2861, 2228, 1652, 1613, 1575, 1495,1472, 1265, 1083,1028 $\mathrm{cm}^{-1}$; HRMS-EI (m/z): $\left[\mathrm{M}^{+}\right]$calcd for $\mathrm{C}_{16} \mathrm{H}_{14} \mathrm{NF}: 238.1032$ found 238.1028.

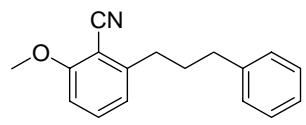

2-Methoxy-6-(3-phenyl-propyl)-benzonitrile (53): Following the general procedure above on $0.2 \mathrm{mmol}$ of 1-iodo-2-methoxybenzene and 1-chloro-3-phenylpropane as the alkyl chloride. The crude product was purified by column chromatography using $30 \%$ dichloromethane in hexane to give $35 \mathrm{mg}$ (74\% Yield) as a clear colorless oil. ${ }^{1} \mathrm{H}$ NMR $\left(\mathrm{CDCl}_{3}, 400 \mathrm{MHz}\right) \delta$ 7.45-7.38 (m, 1H), 7.32-7.25 (m, 2H), 7.22-7.15 (m, 3H), $6.86(\mathrm{~d}$, $1 \mathrm{H}, \mathrm{J}=7.6 \mathrm{~Hz}), 6.79(\mathrm{~d}, 1 \mathrm{H}, \mathrm{J}=8.4 \mathrm{~Hz}), 3.92(\mathrm{~s}, 3 \mathrm{H}), 2.85(\mathrm{~m}, 2 \mathrm{H}), 2.70(\mathrm{~m}, 2 \mathrm{H}), 2.00(\mathrm{~m}$, $2 \mathrm{H}) ;{ }^{13} \mathrm{C}-\mathrm{NMR}\left(\mathrm{CDCl}_{3}, 100 \mathrm{MHz}\right) \delta 161.7,148.2,141.6,133.5,128.3,128.3,125.9$, 121.2, 115.5, 108.4, 102.0, 56.0, 35.4, 34.2, 32.2; IR (film) 2940, 2219, 1643, 1595, 1579, 1494, 1475, 1453, 1436, 1279, 1078, 772, $699 \mathrm{~cm}^{-1}$; HRMS-EI (m/z): $\left[\mathrm{M}^{+}\right] \mathrm{calcd}$ for $\mathrm{C}_{17} \mathrm{H}_{16} \mathrm{NO}$ : 250.1231 found 250.1237 .<smiles>CN(C)c1cccc(CCCc2ccccc2)c1C#N</smiles>

2-Dimethylamino-6-(3-phenyl-propyl)-benzonitrile (54): Following the general procedure above on $0.2 \mathrm{mmol}$ of and 1-chloro-3-phenylpropane as the alkyl chloride and heating for 48 hours. The crude product was purified by column chromatography using $30 \%$ dichloromethane in hexane to give $18 \mathrm{mg}$ (34\% Yield) as a clear colorless oil. ${ }^{1} \mathrm{H}$ NMR $\left(\mathrm{CDCl}_{3}, 400 \mathrm{MHz}\right) \delta$ 7.35-7.25 (m, 3H), 7.24-7.15 (m, 3H), 6.80-6.71 (m, 2H), $2.99(\mathrm{~s}, 6 \mathrm{H}), 2.89-2.81(\mathrm{~m}, 2 \mathrm{H}), 2.76-2.69(\mathrm{~m}, 2 \mathrm{H}), 2.05-1.94(\mathrm{~m}, 2 \mathrm{H}) ;{ }^{13} \mathrm{C}-\mathrm{NMR}$ $\left(\mathrm{CDCl}_{3}, 100 \mathrm{MHz}\right) \delta 157.0,148.2,141.8,132.7,128.4,128.3,125.8,120.5,118.0,114.8$, 103.4, 43.6, 35.5, 34.6, 32.3; IR (film) 3031, 2955, 2872, 2791, 2214, 1736, 1726, 1711, 1599, 1492, 1453,1366, $12551036 \mathrm{~cm}^{-1}$; HRMS-EI (m/z): $\left[\mathrm{M}^{+}\right]$calcd for $\mathrm{C}_{18} \mathrm{H}_{20} \mathrm{~N}_{2}$ : 264.1626 found 266.1620 .

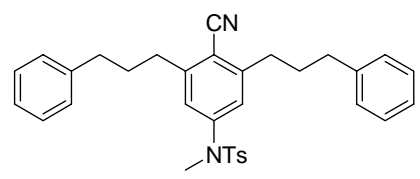

N-[4-Cyano-3,5-bis-(3-phenyl-propyl)-phenyl]-4,N-dimethyl-benzenesulfonamide (55): Following the general procedure above on $0.2 \mathrm{mmol}$ of $\mathrm{N}-(4$-iodophenyl)-4,Ndimethyl-benzenesulfonamide and $1.2 \mathrm{mmol}$ of 1-chloro-3-phenylpropane as the alkyl chloride. The crude product was purified by column chromatography using $10 \%$ ether in hexane to give $41 \mathrm{mg}$ ( $43 \%$ Yield) as a clear colorless oil. ${ }^{1} \mathrm{H}$ NMR $\left(\mathrm{CDCl}_{3}, 400 \mathrm{MHz}\right) \delta$ 7.40-7.35 (m, 2H), 7.32-7.26 (m, 4H), 7.22-7.13 (m, 8H), $6.90(\mathrm{~s}, 2 \mathrm{H}), 3.14(\mathrm{~s}, 3 \mathrm{H}), 2.87-$ $2.78(\mathrm{~m}, 4 \mathrm{H}), 2.69-2.60(\mathrm{~m}, 4 \mathrm{H}), 2.30(\mathrm{~s}, 3 \mathrm{H}), 1.98-1.86(\mathrm{~m}, 4 \mathrm{H}),{ }^{13} \mathrm{C}-\mathrm{NMR}\left(\mathrm{CDCl}_{3}\right.$, $100 \mathrm{MHz}) \delta 147.5,145.0,144.1,141.4,133.1,129.5,128.4,128.3,127.6,126.0,124.0$, 116.6, 110.6, 37.5, 35.4, 34.4, 32.2, 21.4; IR (film) 3059, 3024, 2927, 2860, 2358, 2217 , 1598, 1495, 1453, 1353, 1305, 1185, 1166, 1088, $956 \mathrm{~cm}^{-1}$; HRMS-EI (m/z): [M $]$ calcd for $\mathrm{C}_{33} \mathrm{H}_{34} \mathrm{~N}_{2} \mathrm{O}_{2} \mathrm{~S}$ : 522.2341 found 522.2357 . 


\section{General Procedure for Intermolecular Arylation/Cyanation Reaction} Using Aryl Bromides: To a $5 \mathrm{ml}$ microwave vial containing a magnetic stir bar was added 1.0 equivalent aryl iodide, 1.5 equivalents aryl bromide, 0.025 equivalents palladium acetate, 0.06 equivalents tri(2-furyl)phosphine, 5.5 equivalents $\mathrm{K}_{4}\left[\mathrm{Fe}(\mathrm{CN})_{6}\right] 3 \mathrm{H}_{2} \mathrm{O}, 1.5$ equivalents norbornene and 1.5 equivalents cesium carbonate. The vial was the sealed and flushed with argon. Acetonitrile was added to give a final concentration of $0.05 \mathrm{M}$ based on aryl iodide. The resulting solution was then purged with argon for 5 minutes and stirred for 30 minutes at room temperature, followed by heating with stirring in a microwave at $130{ }^{\circ} \mathrm{C}$ for 2 hours (See below for pressure, temperature and power graphs). The pressure noted at this temperature was 2.5 bar. The mixture was then diluted with water and extracted with dichloromethane. The combined organic phases were dried with sodium sulphate and evaporated under reduced pressure. The product was then purified by flash column chromatography.

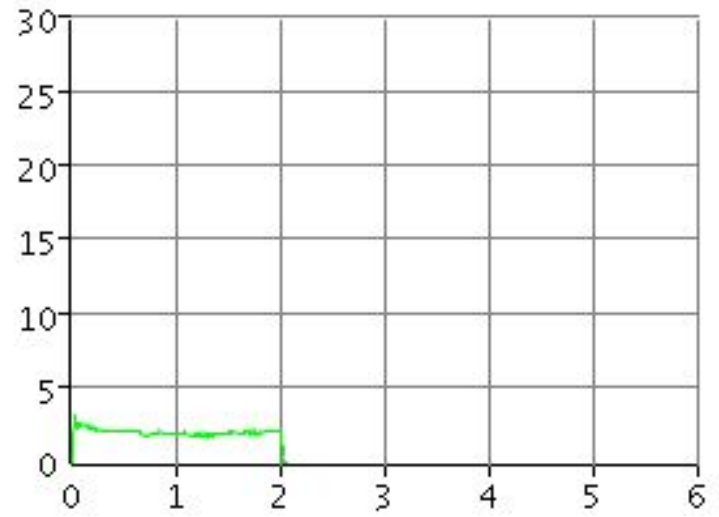

Figure 1: Pressure (bar) Vs Time (hours)

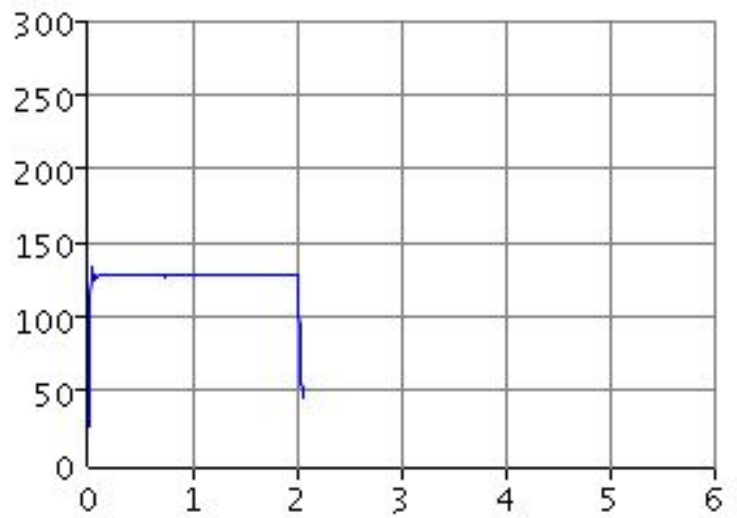

Figure 2: Temperature (degrees celsius) Vs Time (hours) 


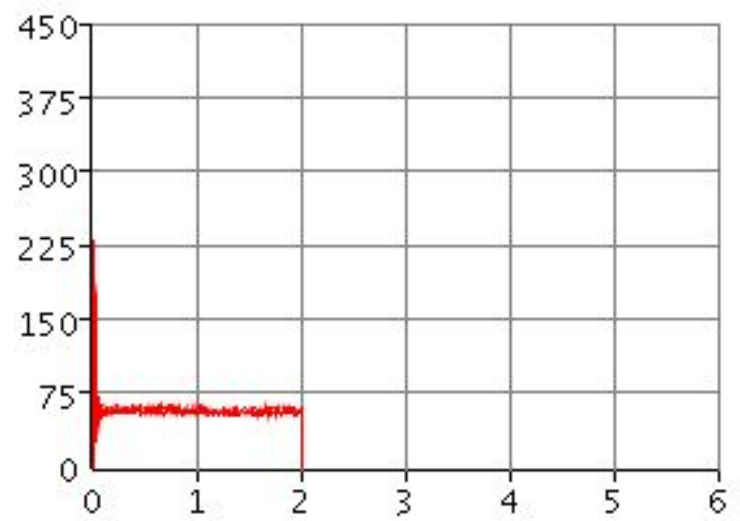

Figure 3: Power (watts) Vs Time (hours)

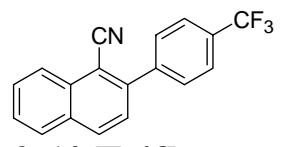

2-(4-Trifluoromethyl-phenyl)-naphthalene-1-carbonitrile (56): Following the general procedure above on $0.2 \mathrm{mmol}$ of iodonaphthalene and 1-bromo-4-trifluoromethylbenzene as the aryl bromide. The crude product was purified by column chromatography using 30\% dichloromethane in hexane to give 39mg (66\% Yield) as a white crystalline solid (mp=91-93). Utilizing 3.0eq. of aryl bromide: $56 \mathrm{mg}$ (94\% Yield). ${ }^{1} \mathrm{H}$ NMR $\left(\mathrm{CDCl}_{3}, 400 \mathrm{MHz}\right) \delta 8.37(\mathrm{ddd}, 1 \mathrm{H}, \mathrm{J}=0.8 \mathrm{~Hz}, \mathrm{~J}=1.8 \mathrm{~Hz}, \mathrm{~J}=8.5 \mathrm{~Hz}), 8.16(\mathrm{~d}, 1 \mathrm{H}, \mathrm{J}=8.4 \mathrm{~Hz})$, $7.98(\mathrm{~d}, 1 \mathrm{H}, \mathrm{J}=8.2 \mathrm{~Hz}), 7.84-7.74(\mathrm{~m}, 5 \mathrm{H}), 7.67(\mathrm{ddd}, 1 \mathrm{H}, \mathrm{J}=1.2 \mathrm{~Hz}, \mathrm{~J}=6.9 \mathrm{~Hz}, \mathrm{~J}=8.1 \mathrm{~Hz})$, $7.60(\mathrm{~d}, 1 \mathrm{H}, \mathrm{J}=8.5 \mathrm{~Hz}),{ }^{19} \mathrm{~F}\left(\mathrm{CDCl}_{3}, 400 \mathrm{MHz}\right) \delta 63.1 ;{ }^{13} \mathrm{C}-\mathrm{NMR}\left(\mathrm{CDCl}_{3}, 100 \mathrm{MHz}\right) \delta$ 144.1, 142.1, 133.2, 132.9, 132.1, 130.9 (d, 32.7Hz), 129.6, 129.2, 128.5, 127.8, 126.6, 125.8 (q, 3.5Hz), 125.7, 122.6, 117.1, 108.4; IR (chloroform solution) 3064, 2931, 2873, $2225,123,1718,1620,1593,1502,1409,1378,1341,1325,1170,1133,1113,1091$, 1067, 1022, 1016, 868, 866, 845, $825 \mathrm{~cm}^{-1}$; HRMS-EI (m/z): $\left[\mathrm{M}^{+}\right]$calcd for $\mathrm{C}_{18} \mathrm{H}_{10} \mathrm{NF}_{3}$ : 297.0765 found 297.0765 .

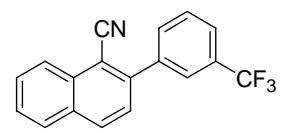

2-(3-Trifluoromethyl-phenyl)-naphthalene-1-carbonitrile (57): Following the general procedure above on $0.2 \mathrm{mmol}$ of iodonaphthalene and 1-bromo-3-trifluoromethylbenzene as the aryl bromide. The crude product was purified by column chromatography using 30\% dichloromethane in hexane to give $18 \mathrm{mg}$ (30\% Yield) as a white crystalline solid (mp=94-95). Utilizing 6.0eq. of aryl bromide: $37 \mathrm{mg}\left(61 \%\right.$ Yield). ${ }^{1} \mathrm{H} \mathrm{NMR}\left(\mathrm{CDCl}_{3}\right.$, $400 \mathrm{MHz}) \delta 8.37(\mathrm{~d}, 1 \mathrm{H}, \mathrm{J}=8.4 \mathrm{~Hz}), 8.16(\mathrm{~d}, 1 \mathrm{H}, \mathrm{J}=8.5 \mathrm{~Hz}), 7.98(\mathrm{~d}, 1 \mathrm{H}, \mathrm{J}=8.1 \mathrm{~Hz}), 7.93-$ $7.88(\mathrm{~m}, 2 \mathrm{H}), 7.8-7.74(\mathrm{~m}, 2 \mathrm{H}), 7.71-7.64(\mathrm{~m}, 2 \mathrm{H}), 7.61(\mathrm{~d}, 1 \mathrm{H}, \mathrm{J}=8.5 \mathrm{~Hz}) ;{ }^{19} \mathrm{~F}\left(\mathrm{CDCl}_{3}\right.$, $400 \mathrm{MHz}) \delta 63.1 ;{ }^{13} \mathrm{C}-\mathrm{NMR}\left(\mathrm{CDCl}_{3}, 100 \mathrm{MHz}\right) \delta 144.1,139.3,133.2,133.0,132.5(\mathrm{~d}$, $1 \mathrm{H}, \mathrm{J}=1.2 \mathrm{~Hz}), 132.0,131.4,131.1,129.3,129.2,128.5,127.8,126.7,126.0(\mathrm{~d}, 1 \mathrm{H}$, $\mathrm{J}=3.7 \mathrm{~Hz}), 125.7,125.6(\mathrm{~d}, 1 \mathrm{H}, \mathrm{J}=3.7 \mathrm{~Hz}), 117.0,108.5$; IR (chloroform solution) 3060 , 2954, 2229, 1593, 1492, 1438, 1376, 1345, 1327, 1275, 1170, 1134, 1073, 909, 830 $\mathrm{cm}^{-1}$; HRMS-EI (m/z): $\left[\mathrm{M}^{+}\right]$calcd for $\mathrm{C}_{18} \mathrm{H}_{10} \mathrm{NF}_{3}$ : 297.0765 found 297.0762 . 


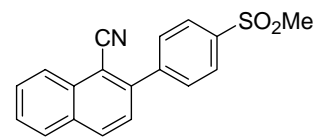

2-(4-Methanesulfonyl-phenyl)-naphthalene-1-carbonitrile (58): Following the general procedure above on $0.2 \mathrm{mmol}$ of iodonaphthalene and 1-bromo-4-methanesulfonylbenzene as the aryl bromide. The crude product was purified by column chromatography using $2.5 \%$ ether in dichloromethane to give $43 \mathrm{mg}$ ( $70 \%$ Yield) as a white crystalline solid (mp=195-197). ${ }^{1} \mathrm{H} \mathrm{NMR}\left(\mathrm{CDCl}_{3}, 400 \mathrm{MHz}\right) \delta 8.37(\mathrm{dd}, 1 \mathrm{H}, \mathrm{J}=0.7 \mathrm{~Hz}, \mathrm{~J}=8.3 \mathrm{~Hz})$, $8.18(\mathrm{~d}, 1 \mathrm{H}, \mathrm{J}=8.4 \mathrm{~Hz}), 8.15-8.10(\mathrm{~m}, 2 \mathrm{H}), 7.99(\mathrm{~d}, 1 \mathrm{H}, \mathrm{J}=8.2 \mathrm{~Hz}), 7.90-7.85(\mathrm{~m}, 2 \mathrm{H}), 7.78$ $(\mathrm{ddd}, 1 \mathrm{H}, \mathrm{J}=1.2 \mathrm{~Hz}, \mathrm{~J}=7.0 \mathrm{~Hz}, \mathrm{~J}=8.3 \mathrm{~Hz}), 7.69(\mathrm{ddd}, 1 \mathrm{H}, \mathrm{J}=1.1 \mathrm{~Hz}, \mathrm{~J}=7.0 \mathrm{~Hz}, \mathrm{~J}=8.1 \mathrm{~Hz}), 7.60$ $(\mathrm{d}, 1 \mathrm{H}, \mathrm{J}=8.5 \mathrm{~Hz}), 3.15(\mathrm{~s}, 3 \mathrm{H}) ;{ }^{13} \mathrm{C}-\mathrm{NMR}\left(\mathrm{CDCl}_{3}, 100 \mathrm{MHz}\right) \delta 144.0,143.5,140.7,133.4$, $132.9,132.2,130.2,129.4,128.5,128.0,127.9,126.5,125.7,116.9,108.5,44.5$; IR ( chloroform solution) 3034, 2960, 2929, 2872, 2235, 1593, 1402, 1320, 1152, 1088, 956, $823 \mathrm{~cm}^{-1}$; HRMS-EI (m/z): $\left[\mathrm{M}^{+}\right]$calcd for $\mathrm{C}_{18} \mathrm{H}_{13} \mathrm{NO}_{2} \mathrm{~S}: 307.0667$ found 307.0664 .

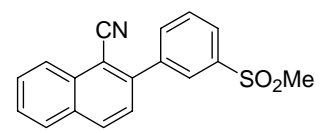

2-(3-Methanesulfonyl-phenyl)-naphthalene-1-carbonitrile (59): Following the general procedure above on $0.2 \mathrm{mmol}$ of iodonaphthalene and 3.0eq. of 1-bromo-3-

methanesulfonyl-benzene as the aryl bromide. The crude product was purified by column chromatography using $2.5 \%$ ether in dichloromethane to give $33 \mathrm{mg}$ (54\% Yield) as a white crystalline solid (mp=151-152). ${ }^{1} \mathrm{H} \mathrm{NMR}\left(\mathrm{CDCl}_{3}, 400 \mathrm{MHz}\right) \delta 8.35(\mathrm{dd}, 1 \mathrm{H}$, $\mathrm{J}=0.8 \mathrm{~Hz}, \mathrm{~J}=8.4 \mathrm{~Hz}), 8.26(\mathrm{t}, 1 \mathrm{H}, \mathrm{J}=1.6 \mathrm{~Hz}), 8.18(\mathrm{~d}, 1 \mathrm{H}, \mathrm{J}=8.5 \mathrm{~Hz}), 8.09$ (ddd, $1 \mathrm{H}$, $\mathrm{J}=1.1 \mathrm{~Hz}, \mathrm{~J}=1.8 \mathrm{~Hz}, \mathrm{~J}=7.9 \mathrm{~Hz}), 7.99(\mathrm{ddd}, 2 \mathrm{H}, \mathrm{J}=1.8 \mathrm{~Hz}, \mathrm{~J}=3.0 \mathrm{~Hz}, \mathrm{~J}=4.4 \mathrm{~Hz}), 7.81-7.72(\mathrm{~m}$, 2H), $7.68(\mathrm{ddd}, 1 \mathrm{H}, \mathrm{J}=1.1 \mathrm{~Hz}, \mathrm{~J}=7.0 \mathrm{~Hz}, \mathrm{~J}=8.1 \mathrm{~Hz}), 7.63(\mathrm{~m}, 1 \mathrm{H}), 3.16(\mathrm{~s}, 3 \mathrm{H}) ;{ }^{13} \mathrm{C}-\mathrm{NMR}$ $\left(\mathrm{CDCl}_{3}, 100 \mathrm{MHz}\right) \delta 143.4,141.2,140.0,134.3,133.5,132.9,132.2,129.9,129.4,128.6$, 128.2, 128.0, 127.5, 126.4, 125.7, 117.2, 108.6, 44.6; IR ( chloroform solution) 3031, 2931, 2362, 2226, 1592, 1484, 1408, 137, 1321, 1305, 1190, 960, $829 \mathrm{~cm}^{-1}$; HRMS-EI $(\mathrm{m} / \mathrm{z}):\left[\mathrm{M}^{+}\right]$calcd for $\mathrm{C}_{18} \mathrm{H}_{13} \mathrm{NO}_{2} \mathrm{~S}: 307.0667$ found 307.0671 .

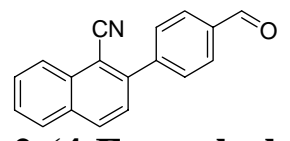

2-(4-Formyl-phenyl)-naphthalene-1-carbonitrile (60): Following the general procedure above on $0.2 \mathrm{mmol}$ of iodonaphthalene and 3.0eq. of 4-bromo-benzaldehyde as the aryl bromide. The crude product was purified by column chromatography using $50 \%$ dichloromethane in hexane to give $24 \mathrm{mg}$ (51\% Yield) as a white crystalline solid $(\mathrm{mp}=200-201) .{ }^{1} \mathrm{H} \mathrm{NMR}\left(\mathrm{CDCl}_{3}, 400 \mathrm{MHz}\right) \delta 10.13(\mathrm{~s}, 1 \mathrm{H}), 8.37(\mathrm{dd}, 1 \mathrm{H}, \mathrm{J}=0.9 \mathrm{~Hz}$, $\mathrm{J}=8.4 \mathrm{~Hz}), 8.16(\mathrm{~m}, 1 \mathrm{H}), 8.08-8.04(\mathrm{~m}, 2 \mathrm{H}), 7.98(\mathrm{~d}, 1 \mathrm{H}, \mathrm{J}=8.2 \mathrm{~Hz}), 7.88-7.83(\mathrm{~m}, 2 \mathrm{H})$, 7.77 (ddd, $1 \mathrm{H}, \mathrm{J}=1.3 \mathrm{~Hz}, \mathrm{~J}=6.9 \mathrm{~Hz}, \mathrm{~J}=8.4 \mathrm{~Hz}), 7.68$ (ddd, $1 \mathrm{H}, \mathrm{J}=1.2 \mathrm{~Hz}, \mathrm{~J}=7.0 \mathrm{~Hz}, \mathrm{~J}=8.1 \mathrm{~Hz}$ ), $7.62(\mathrm{~d}, 1 \mathrm{H}, \mathrm{J}=8.5 \mathrm{~Hz}) ;{ }^{13} \mathrm{C}-\mathrm{NMR}\left(\mathrm{CDCl}_{3}, 100 \mathrm{MHz}\right) \delta 191.9,144.6,144.4,136.4,133.4$, 133.2, 132.4, 130.2, 130.1, 129.5, 128.7, 128.1, 126.7, 125.9, 117.3, 108.6; IR ( chloroform solution) 3063, 2965, 2835, 2745, 2226, 1708, 1705, 1693, 1608, 1593, 1503, $1378,1307,1171,1014,819 \mathrm{~cm}^{-1}$; HRMS-EI (m/z): $\left[\mathrm{M}^{+}\right]$calcd for $\mathrm{C}_{18} \mathrm{H}_{11} \mathrm{NO}: 257.0840$ found 257.0834 . 


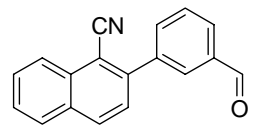

2-(3-Formyl-phenyl)-naphthalene-1-carbonitrile (61): Following the general procedure above on $0.2 \mathrm{mmol}$ of iodonaphthalene and 3-bromo-benzaldehyde as the aryl bromide. The crude product was purified by column chromatography using $50 \%$ dichloromethane in hexane to give $14 \mathrm{mg}$ ( $26 \%$ Yield) as a white crystalline solid $(\mathrm{mp}=163-164)$. Utilizing 6.0eq. of aryl bromide: $25 \mathrm{mg}\left(47 \%\right.$ Yield). ${ }^{1} \mathrm{H} \mathrm{NMR}\left(\mathrm{CDCl}_{3}\right.$, $400 \mathrm{MHz}) \delta 10.14(\mathrm{~s}, 1 \mathrm{H}), 8.37(\mathrm{dd}, 1 \mathrm{H}, \mathrm{J}=0.9 \mathrm{~Hz}, \mathrm{~J}=8.4 \mathrm{~Hz}), 8.19-8.14(\mathrm{~m}, 2 \mathrm{H}, \mathrm{J}=3.1 \mathrm{~Hz}$, $\mathrm{J}=4.7 \mathrm{~Hz}), 8.04-7.96(\mathrm{~m}, 3 \mathrm{H}), 7.80-7.71(\mathrm{~m}, 2 \mathrm{H}), 7.67(\mathrm{ddd}, 1 \mathrm{H}, \mathrm{J}=1.2 \mathrm{~Hz}, \mathrm{~J}=6.9 \mathrm{~Hz}$, $\mathrm{J}=8.1 \mathrm{~Hz}), 7.63(\mathrm{~d}, 1 \mathrm{H}, \mathrm{J}=8.5 \mathrm{~Hz}) ;{ }^{13} \mathrm{C}-\mathrm{NMR}\left(\mathrm{CDCl}_{3}, 100 \mathrm{MHz}\right) \delta 191.7,144.2,139.6$, 136.8, 135.0, 133.2, 133.0, 132.0, 130.3, 129.9, 129.5, 129.2, 128.5, 127.7, 126.7, 125.7, 117.1, 108.4; IR ( chloroform solution) 3033, 2835, 2227, 1844, 1772, 1707, 1702, 1698, 1693, 1583, 1187, 1167, 1156, $832 \mathrm{~cm}^{-1}$; HRMS-EI (m/z): $\left[\mathrm{M}^{+}\right]$calcd for $\mathrm{C}_{18} \mathrm{H}_{11} \mathrm{NO}$ : 257.0840 found 257.0840 .

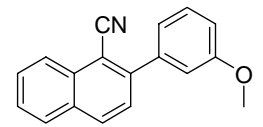

2-(3-Methoxy-phenyl)-naphthalene-1-carbonitrile (63): Following the general procedure above on $0.2 \mathrm{mmol}$ of iodonaphthalene and $2.3 \mathrm{mmol}$ of 1-bromo-3-methoxybenzene as the aryl bromide. The crude product was purified by column chromatography using $30 \%$ dichloromethane in hexane to give $29 \mathrm{mg}(56 \%$ Yield) as a white crystalline solid(mp=97-98). ${ }^{1} \mathrm{H} \mathrm{NMR}\left(\mathrm{CDCl}_{3}, 400 \mathrm{MHz}\right) \delta 8.36(\mathrm{dd}, 1 \mathrm{H}, \mathrm{J}=0.9 \mathrm{~Hz}, \mathrm{~J}=8.4 \mathrm{~Hz}), 8.10$ $(\mathrm{d}, 1 \mathrm{H}, \mathrm{J}=8.5 \mathrm{~Hz}), 7.95(\mathrm{~d}, 1 \mathrm{H}, \mathrm{J}=8.2 \mathrm{~Hz}), 7.73(\mathrm{ddd}, 1 \mathrm{H}, \mathrm{J}=1.3 \mathrm{~Hz}, \mathrm{~J}=6.9 \mathrm{~Hz}, \mathrm{~J}=8.4 \mathrm{~Hz})$, 7.66-7.59 (m, 2H), 7.48-7.42 (m, 1H), 7.28-7.20(m, 2H), 7.03 (ddd, 1H, J=0.9Hz, $\mathrm{J}=2.6 \mathrm{~Hz}, \mathrm{~J}=8.3 \mathrm{~Hz}), 3.90(\mathrm{~s}, 3 \mathrm{H}) ;{ }^{13} \mathrm{C}-\mathrm{NMR}\left(\mathrm{CDCl}_{3}, 100 \mathrm{MHz}\right) \delta 159.9,145.9,140.1$, 133.3, 133.0, 132.0, 130.0, 129.1, 128.6, 127.6, 127.2, 125.9, 121.8, 117.6, 114.9, 114.8, 108.3, 55.6; IR ( chloroform solution) 2837, 2228, 1593, 1492, 1466, 1463, 1293, 1037 ,

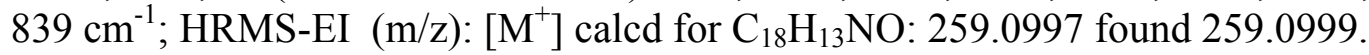

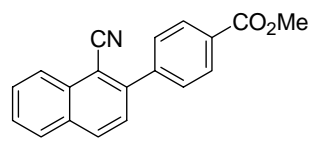

4-(1-Cyano-naphthalen-2-yl)-benzoic acid methyl ester (64): Following the general procedure above on $0.2 \mathrm{mmol}$ of iodonaphthalene and 4-bromo-benzoic acid methyl ester as the aryl bromide. The crude product was purified by column chromatography using $50 \%$ dichloromethane in hexane to give $31 \mathrm{mg}$ (54\% Yield) as a white crystalline $\operatorname{solid}(\mathrm{mp}=186-187) .{ }^{1} \mathrm{H} \mathrm{NMR}\left(\mathrm{CDCl}_{3}, 400 \mathrm{MHz}\right) \delta 8.36(\mathrm{dd}, 1 \mathrm{H}, \mathrm{J}=0.7 \mathrm{~Hz}, \mathrm{~J}=8.4 \mathrm{~Hz})$, 8.23-8.18 (m, 2H), 8.14 (d, 1H, J=8.5Hz), $7.97(\mathrm{~d}, 1 \mathrm{H}, \mathrm{J}=8.1 \mathrm{~Hz}), 7.78-7.72(\mathrm{~m}, 3 \mathrm{H}), 7.66$ (ddd, $1 \mathrm{H}, \mathrm{J}=1.1 \mathrm{~Hz}, \mathrm{~J}=7.0 \mathrm{~Hz}, \mathrm{~J}=8.1 \mathrm{~Hz}), 7.61(\mathrm{~d}, 1 \mathrm{H}, \mathrm{J}=8.5 \mathrm{~Hz}), 3.97(\mathrm{~s}, 3 \mathrm{H}) ;{ }^{13} \mathrm{C}-\mathrm{NMR}$ $\left(\mathrm{CDCl}_{3}, 100 \mathrm{MHz}\right) \delta 166.8,144.8,143.2,133.3,133.2,132.3,130.6,130.2,129.5,129.4$, 128.7, 127.9, 126.9, 125.9, 117.3, 108.5, 52.5; IR (chloroform solution) 3002, 2956, 2227, 1736, 1724, 1721, 1711, 1613, 1593, 1501, 1438, 1406, 1378, 1314, 1286, 1183, 1117, 1016, $828 \mathrm{~cm}^{-1}$; HRMS-EI (m/z): $\left[\mathrm{M}^{+}\right]$calcd for $\mathrm{C}_{19} \mathrm{H}_{13} \mathrm{NO}_{2}: 288.1019$ found 288.1019 . 


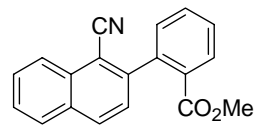

2-(1-Cyano-naphthalen-2-yl)-benzoic acid methyl ester (65): Following the general procedure above on $0.2 \mathrm{mmol}$ of iodonaphthalene and 2-bromo-benzoic acid methyl ester as the aryl bromide. The crude product was purified by column chromatography using $50 \%$ dichloromethane in hexane to give $16 \mathrm{mg}$ (28\% Yield) as a white crystalline solid $(\mathrm{mp}=129-130) .{ }^{1} \mathrm{H} \mathrm{NMR}\left(\mathrm{CDCl}_{3}, 300 \mathrm{MHz}\right) \delta 8.29(\mathrm{~d}, 1 \mathrm{H}, \mathrm{J}=8.1 \mathrm{~Hz}), 8.14(\mathrm{dd}, 1 \mathrm{H}$, $\mathrm{J}=1.3 \mathrm{~Hz}, \mathrm{~J}=7.8 \mathrm{~Hz}), 8.07(\mathrm{~d}, 1 \mathrm{H}, \mathrm{J}=8.5 \mathrm{~Hz}), 7.96(\mathrm{~d}, 1 \mathrm{H}, \mathrm{J}=8.0 \mathrm{~Hz}), 7.78-7.52(\mathrm{~m}, 4 \mathrm{H})$, 7.48-7.40 (m, 2H), 3.66 (s, 3H); ${ }^{13} \mathrm{C}-\mathrm{NMR}\left(\mathrm{CDCl}_{3}, \mathrm{MHz}\right) \delta 166.8,146.4,140.1,132.4$, $132.1,131.9,131.8,131.2$, 130.9, 129.6, 128.8, 128.7, 128.5, 127.3, 126.9, 125.5, 116.9, 108.8, 52.1; IR (film) 3058, 2949, 2221, 1725, 1593, 1491, 1433, 1287, 1264, 1190, 1137, 1102, 1073, 965, 829, 763, 712, $620 \mathrm{~cm}^{-1}$; HRMS-ESI (m/z): $\left[\mathrm{M}^{+}\right]$calcd for $\left[\mathrm{C}_{19} \mathrm{H}_{13} \mathrm{NO}_{2} \mathrm{Na}\right]^{+}: 310.0838$ found 310.0842 . Reoptimized conditions: iodonaphthalene (1.0 equiv), aryl bromide (1.0 equiv), $\mathrm{Pd}(\mathrm{OAc})_{2}(2.5 \mathrm{~mol} \%)$, triphenylphosphine (6 mol\%), $\mathrm{Cs}_{2} \mathrm{CO}_{3}$ (1.5 equiv), norbornene (0.5 equiv), and anhydrous $\mathrm{K}_{4}\left[\mathrm{Fe}(\mathrm{CN})_{6}\right](5.5$ equiv) in $\mathrm{CH}_{3} \mathrm{CN}(0.05 \mathrm{M})$ were heated in a sealed tube at $90{ }^{\circ} \mathrm{C}$ for $24 \mathrm{~h}$ under conventional heating yielding 46mg (81\% Yield).

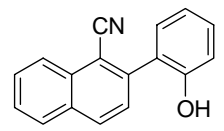

2-(2-Hydroxy-phenyl)-naphthalene-1-carbonitrile (66): Following the general procedure above on $0.2 \mathrm{mmol}$ of iodonaphthalene and 2-bromophenol as the aryl bromide. The crude product was purified by column chromatography using $20 \%$ dichloromethane and $20 \%$ ether in hexane to give $19 \mathrm{mg}(39 \%$ Yield) as a beige solid $(\mathrm{mp}=134-135) .{ }^{1} \mathrm{H} \mathrm{NMR}\left(\mathrm{CDCl}_{3}, 400 \mathrm{MHz}\right) \delta 8.34(\mathrm{dd}, 1 \mathrm{H}, \mathrm{J}=0.8 \mathrm{~Hz}, \mathrm{~J}=8.4 \mathrm{~Hz}), 8.13(\mathrm{~d}$, $1 \mathrm{H}, \mathrm{J}=8.5 \mathrm{~Hz}), 7.97(\mathrm{~d}, 1 \mathrm{H}, \mathrm{J}=8.2 \mathrm{~Hz}), 7.74(\mathrm{ddd}, 1 \mathrm{H}, \mathrm{J}=1.3 \mathrm{~Hz}, \mathrm{~J}=6.9 \mathrm{~Hz}, \mathrm{~J}=8.4 \mathrm{~Hz}), 7.65$ (ddd, $1 \mathrm{H}, \mathrm{J}=1.2 \mathrm{~Hz}, \mathrm{~J}=6.9 \mathrm{~Hz}, \mathrm{~J}=8.1 \mathrm{~Hz}), 7.60(\mathrm{~d}, 1 \mathrm{H}, \mathrm{J}=8.5 \mathrm{~Hz}), 7.42-7.34(\mathrm{~m}, 2 \mathrm{H}), 7.10$ $(\mathrm{dt}, 1 \mathrm{H}, \mathrm{J}=1.1 \mathrm{~Hz}, \mathrm{~J}=7.5 \mathrm{~Hz}), 7.01(\mathrm{dd}, 1 \mathrm{H}, \mathrm{J}=0.8 \mathrm{~Hz}, \mathrm{~J}=8.2 \mathrm{~Hz}), 4.92(\mathrm{~s}, 1 \mathrm{H}) ;{ }^{13} \mathrm{C}-\mathrm{NMR}$ $\left(\mathrm{CDCl}_{3}, 100 \mathrm{MHz}\right) \delta 152.4,141.9,132.8,132.0,131.1,130.6,128.9,128.4,127.6,127.5$, 126.7, 125.6, 123.2, 121.2, 118.8, 116.4, 110.3; IR (chloroform solution) 3575, 3300, 2926, 2357, 2230, 1700, 1611, 1589, 1502, 1450, 1325, 1286, 1175, $1112 \mathrm{~cm}^{-1}$; HRMS$\mathrm{EI}(\mathrm{m} / \mathrm{z}):\left[\mathrm{M}^{+}\right]$calcd for $\mathrm{C}_{17} \mathrm{H}_{11} \mathrm{NO}: 245.0840$ found 235.0840 .

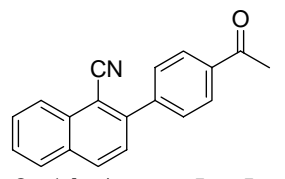

2-(4-Acetyl-phenyl)-naphthalene-1-carbonitrile (67): Following the general procedure above on $0.2 \mathrm{mmol}$ of iodonaphthalene and 1-(4-bromophenyl)-ethanone as the aryl bromide. The crude product was purified by column chromatography using $50 \%$ dichloromethane in hexane to give $33 \mathrm{mg}(61 \%$ Yield) as a white crystalline solid $(\mathrm{mp}=166-169) .{ }^{1} \mathrm{H} \mathrm{NMR}\left(\mathrm{CDCl}_{3}, 400 \mathrm{MHz}\right) \delta 8.37(\mathrm{dd}, 1 \mathrm{H}, \mathrm{J}=0.9 \mathrm{~Hz}, \mathrm{~J}=8.4 \mathrm{~Hz}), 8.18$ $8.10(\mathrm{~m}, 3 \mathrm{H}), 7.98(\mathrm{~d}, 1 \mathrm{H}, \mathrm{J}=8.1 \mathrm{~Hz}), 7.81-7.73(\mathrm{~m}, 3 \mathrm{H}), 7.67(\mathrm{ddd}, 1 \mathrm{H}, \mathrm{J}=1.2 \mathrm{~Hz}$, $\mathrm{J}=6.9 \mathrm{~Hz}, \mathrm{~J}=8.1 \mathrm{~Hz}), 7.62(\mathrm{~d}, 1 \mathrm{H}, \mathrm{J}=8.5 \mathrm{~Hz}), 2.68(\mathrm{~s}, 3 \mathrm{H}) ;{ }^{13} \mathrm{C}-\mathrm{NMR}\left(\mathrm{CDCl}_{3}, 100 \mathrm{MHz}\right)$ $\delta 197.5,144.5,143.1,137.0,133.1,133.0,132.1,129.5,129.2,128.7,128.5,127.7,126.6$, 
125.7, 117.1, 108.3, 26.7; IR (chloroform solution) 3063, 3002, 2227, 1686, 1681, 1608, 1594, 1359, 1267, 1013, $823 \mathrm{~cm}^{-1}$; HRMS-EI (m/z): [M $]$ calcd for $\mathrm{C}_{19} \mathrm{H}_{13} \mathrm{NO}: 271.0997$ found 271.0992 .

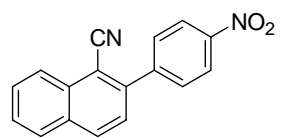

2-(4-Nitro-phenyl)-naphthalene-1-carbonitrile (68): Following the general procedure above on $0.2 \mathrm{mmol}$ of iodonaphthalene and 1-bromo-4-nitrobenzene as the aryl bromide. The crude product was purified by column chromatography using $50 \%$ dichloromethane in hexane to give $50 \mathrm{mg}$ (91\% Yield) as a yellow crystalline solid (mp=203-204). ${ }^{1} \mathrm{H}$ NMR $\left(\mathrm{CDCl}_{3}, 400 \mathrm{MHz}\right) \delta 8.43-8.37$ (m, 2H), 8.37 (dd, $\left.1 \mathrm{H}, \mathrm{J}=0.9 \mathrm{~Hz}, \mathrm{~J}=8.4 \mathrm{~Hz}\right), 8.19$ (d, $1 \mathrm{H}, \mathrm{J}=8.4 \mathrm{~Hz}), 8.00(\mathrm{~d}, 1 \mathrm{H}, \mathrm{J}=8.2 \mathrm{~Hz}), 7.88-7.82(\mathrm{~m}, 2 \mathrm{H}), 7.79$ (ddd, $1 \mathrm{H}, \mathrm{J}=1.3 \mathrm{~Hz}$, $\mathrm{J}=6.9 \mathrm{~Hz}, \mathrm{~J}=8.4 \mathrm{~Hz}$ ), 7.70 (ddd, $1 \mathrm{H}, \mathrm{J}=1.2 \mathrm{~Hz}, \mathrm{~J}=7.0 \mathrm{~Hz}, \mathrm{~J}=8.1 \mathrm{~Hz}), 7.61(\mathrm{~d}, 1 \mathrm{H}, \mathrm{J}=8.5 \mathrm{~Hz}$ ); ${ }^{13} \mathrm{C}-\mathrm{NMR}\left(\mathrm{CDCl}_{3}, 75 \mathrm{MHz}\right) \delta 148.0,144.9,143.1,133.4,132.9,132.3,130.2,129.5$, 128.5, 128.1 126.3, 125.8, 124.0, 116.8, 108.6; IR (chloroform solution) 3033, 2961, $2873,2228,1931,1700,1684,1604,1535,1530,1524,1378,1350,1312,1108,858$, 843, $826 \mathrm{~cm}^{-1}$; HRMS-EI (m/z): $\left[\mathrm{M}^{+}\right]$calcd for $\mathrm{C}_{17} \mathrm{H}_{10} \mathrm{~N}_{2} \mathrm{O}_{2}: 274.0742$ found 274.0744.

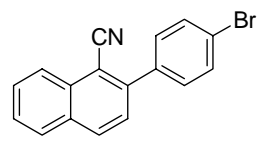

2-(4-Bromo-phenyl)-naphthalene-1-carbonitrile (69): Following the general procedure above on $0.2 \mathrm{mmol}$ of iodonaphthalene and 1,4-dibromobenzene as the aryl bromide. The crude product was purified by column chromatography using $20 \%$ dichloromethane in hexane to give $14 \mathrm{mg}(21 \%$ Yield) as a white crystalline solid ( $\mathrm{mp}=167)$. Utilizing 6.0 eq. aryl bromide: $36 \mathrm{mg}\left(56 \%\right.$ Yield). ${ }^{1} \mathrm{H} \mathrm{NMR}\left(\mathrm{CDCl}_{3}, 400 \mathrm{MHz}\right) \delta 8.35(\mathrm{dd}, 1 \mathrm{H}, \mathrm{J}=0.9 \mathrm{~Hz}$, $\mathrm{J}=8.4 \mathrm{~Hz}), 8.12(\mathrm{~d}, 1 \mathrm{H}, \mathrm{J}=8.5 \mathrm{~Hz}), 7.96(\mathrm{~d}, 1 \mathrm{H}, \mathrm{J}=8.2 \mathrm{~Hz}), 7.75(\mathrm{ddd}, 1 \mathrm{H}, \mathrm{J}=1.3 \mathrm{~Hz}$, $\mathrm{J}=6.9 \mathrm{~Hz}, \mathrm{~J}=8.3 \mathrm{~Hz}), 7.70-7.62(\mathrm{~m}, 3 \mathrm{H}), 7.64-7.52(\mathrm{~m}, 3 \mathrm{H}) ;{ }^{13} \mathrm{C}-\mathrm{NMR}\left(\mathrm{CDCl}_{3}, 100 \mathrm{MHz}\right)$ 8144.7, 137.7, 133.3 133.2, 132.2, 132.1, 130.9, 129.3, 128.6, 127.8, 126.9, 125.8, 123.7, 117.5, 108.3; IR (chloroform solution) 3063, 3006, 2955, 2874, 2225, 1621, 1596, 1493,

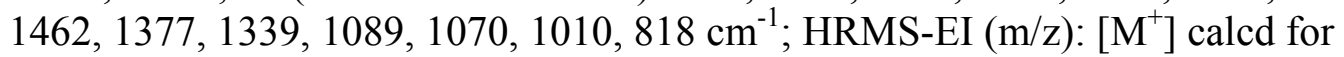
$\mathrm{C}_{17} \mathrm{H}_{10} \mathrm{NBr}$ : 306.9996 found 306.9999 .

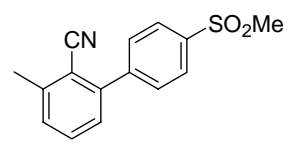

4'-Methanesulfonyl-3-methyl-biphenyl-2-carbonitrile (70): Following the general procedure above on $0.2 \mathrm{mmol}$ of 1-iodo-2-methylbenzene and 4-bromophenyl methylsulfone as the aryl bromide. The crude product was purified by column chromatography using $2.5 \%$ ether in dichloromethane to give $42 \mathrm{mg}$ ( $78 \%$ Yield) as a white solid (mp=171-173). ${ }^{1} \mathrm{H} \mathrm{NMR}\left(\mathrm{CDCl}_{3}, 400 \mathrm{MHz}\right) \delta 8.09-8.03(\mathrm{~m}, 2 \mathrm{H}), 7.77-7.71$ $(\mathrm{m}, 2 \mathrm{H}), 7.57$ (t, $1 \mathrm{H}, \mathrm{J}=7.8 \mathrm{~Hz}), 7.40$ (d, 1H, J=7.8Hz), 7.32 (d, 1H, J=7.8Hz), 3.13 (s, $3 \mathrm{H}), 2.65(\mathrm{~s}, 3 \mathrm{H}) ;{ }^{13} \mathrm{C}-\mathrm{NMR}\left(\mathrm{CDCl}_{3}, 100 \mathrm{MHz}\right) \delta 144.0,143.7,143.5,140.5,132.5$, 129.9, 129.8, 127.7, 127.3, 117.1, 111.7, 44.5, 21.1; IR (chloroform solution) 3033, 2956, 2927, 2855, 2224, 1590, 1466, 1392, 1321, 1304, 1152, 1087, 1019, 957, $842 \mathrm{~cm}^{-1}$; HRMS-EI (m/z): $\left[\mathrm{M}^{+}\right]$calcd for $\mathrm{C}_{15} \mathrm{H}_{13} \mathrm{NO}_{2} \mathrm{~S}: 271.0667$ found 271.0666 . 


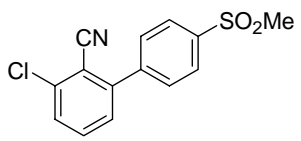

3-Chloro-4'-methanesulfonyl-biphenyl-2-carbonitrile (71): Following the general procedure above on $0.2 \mathrm{mmol}$ of 1-chloro-2-iodobenzene and 4-bromophenyl methylsulfone as the aryl bromide. The crude product was purified by column chromatography using $2.5 \%$ ether in dichloromethane to give $18 \mathrm{mg}(31 \%$ Yield) as a white solid $(\mathrm{mp}=177-178) .{ }^{1} \mathrm{H} \mathrm{NMR}\left(\mathrm{CDCl}_{3}, 400 \mathrm{MHz}\right) \delta 8.12-8.07(\mathrm{~m}, 2 \mathrm{H}), 7.78-7.73$ (m, 2H), 7.67-7.58 (m, 2H), $7.41(\mathrm{dd}, 1 \mathrm{H}, \mathrm{J}=1.6 \mathrm{~Hz}, \mathrm{~J}=7.2 \mathrm{~Hz}), 3.13(\mathrm{~s}, 3 \mathrm{H}),{ }^{13} \mathrm{C}-\mathrm{NMR}$ $\left(\mathrm{CDCl}_{3}, 100 \mathrm{MHz}\right) \delta 145.6,142.7,141.1,138.5,133.5,129.8,129.6,128.1,127.9,115.1$, 112.4, 44.5; IR (chloroform solution) 3016, 2927, 2362, 2236, 1557, 1453, 1319, 1151, 1091, $956 \mathrm{~cm}^{-1}$; HRMS-EI (m/z): [M $\left.{ }^{+}\right]$calcd for $\mathrm{C}_{14} \mathrm{H}_{10} \mathrm{NO}_{2} \mathrm{SCl}: 290.0120$ found 290.0117.

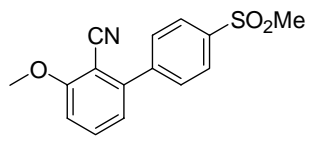

4'-Methanesulfonyl-3-methoxy-biphenyl-2-carbonitrile (72): Following the general procedure above on $0.2 \mathrm{mmol}$ of 1-iodo-2-methoxybenzene and 4-bromophenyl methylsulfone as the aryl bromide. The crude product was purified by column chromatography using 5\% ether in dichloromethane to give $32 \mathrm{mg}$ (54\% Yield) as a white solid $(\mathrm{mp}=204-206) .{ }^{1} \mathrm{H}$ NMR $\left(\mathrm{CDCl}_{3}, 400 \mathrm{MHz}\right) \delta 8.08-8.02(\mathrm{~m}, 2 \mathrm{H}), 7.78-7.72(\mathrm{~m}$, 2H), 7.66-7.06 (m, 1H), 7.09-7.03 (m, 2H), $4.01(\mathrm{~s}, 3 \mathrm{H}), 3.12(\mathrm{~s}, 3 \mathrm{H}) ;{ }^{13} \mathrm{C}-\mathrm{NMR}$ $\left(\mathrm{CDCl}_{3}, 100 \mathrm{MHz}\right) \delta 162.5,145.2,143.6,140.8,134.4,130.0,127.9,122.0,115.7,111.0$, 101.1, 56.6, 44.7; IR (chloroform solution) 3039, 2947, 2843, 2226, 1581, 1574, 1566, 1470, 1474, 1317, 1281, 1266, 1153, 1122, 1015, 957, $841 \mathrm{~cm}^{-1}$; HRMS-EI (m/z): [M ] calcd for $\mathrm{C}_{15} \mathrm{H}_{13} \mathrm{NO}_{2} \mathrm{~S}: 287.0616$ found 287.0613 .

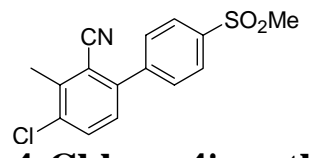

4-Chloro-4'-methanesulfonyl-3-methyl-biphenyl-2-carbonitrile (73): Following the general procedure above on $0.2 \mathrm{mmol}$ of 1-chloro-3-iodo-2-methylbenezene and 4bromophenyl methylsulfone as the aryl bromide. The crude product was purified by column chromatography using $2.5 \%$ ether in dichloromethane to give $38 \mathrm{mg}$ (62\% Yield) as a white solid $(\mathrm{mp}=110-112) .{ }^{1} \mathrm{H} \mathrm{NMR}\left(\mathrm{CDCl}_{3}, 400 \mathrm{MHz}\right) \delta 8.10-8.04(\mathrm{~m}, 2 \mathrm{H}), 7.75-$ $7.68(\mathrm{~m}, 2 \mathrm{H}), 7.67(\mathrm{~d}, 1 \mathrm{H}, \mathrm{J}=8.3 \mathrm{~Hz}), 7.28(\mathrm{dd}, 1 \mathrm{H}, \mathrm{J}=0.5 \mathrm{~Hz}, \mathrm{~J}=8.4 \mathrm{~Hz}), 3.13(\mathrm{~s}, 3 \mathrm{H}), 2.71$ $(\mathrm{s}, 3 \mathrm{H}) ;{ }^{13} \mathrm{C}-\mathrm{NMR}\left(\mathrm{CDCl}_{3}, 100 \mathrm{MHz}\right) \delta 143.1,142.5,141.2,140.8,135.5,133.5,129.8$, 128.3, 127.8, 116.5, 113.6, 44.5, 19.2; IR (chloroform solution) 3032, 2923, 2853, 2227 , 1844, 1670, 1652, 1560, 1462, 1321, 1153, 1096, 1017, $957 \mathrm{~cm}^{-1}$; HRMS-EI (m/z): [M ] calcd for $\mathrm{C}_{15} \mathrm{H}_{12} \mathrm{NO}_{2} \mathrm{SCl}$ : 305.0277 found 305.0279 . 


\section{Spectra}

2-Butyl-naphthalene-1-carbonitrile (25)
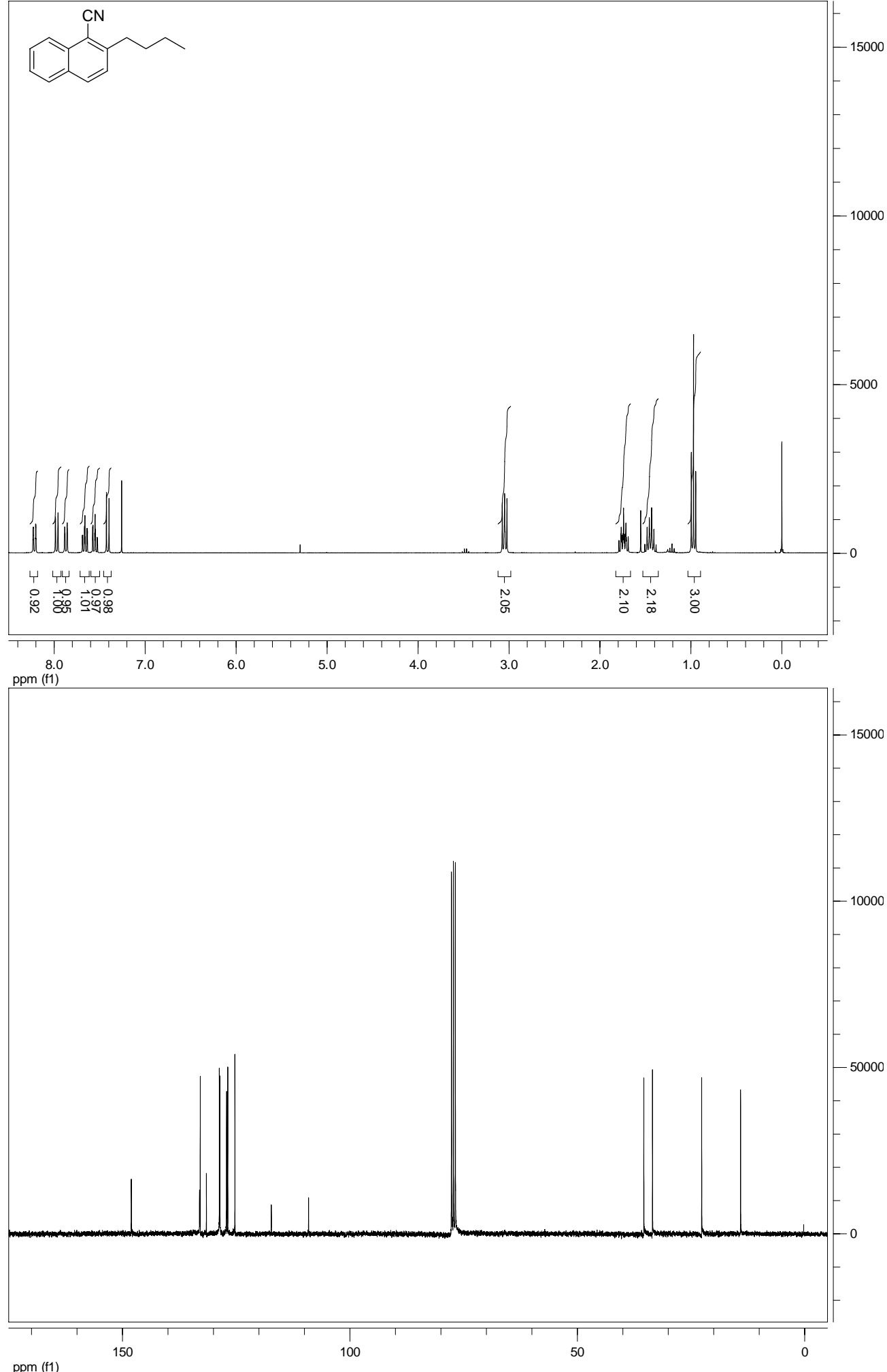

ppm (f1) 
2-Butyl-6-methyl-benzonitrile (26)

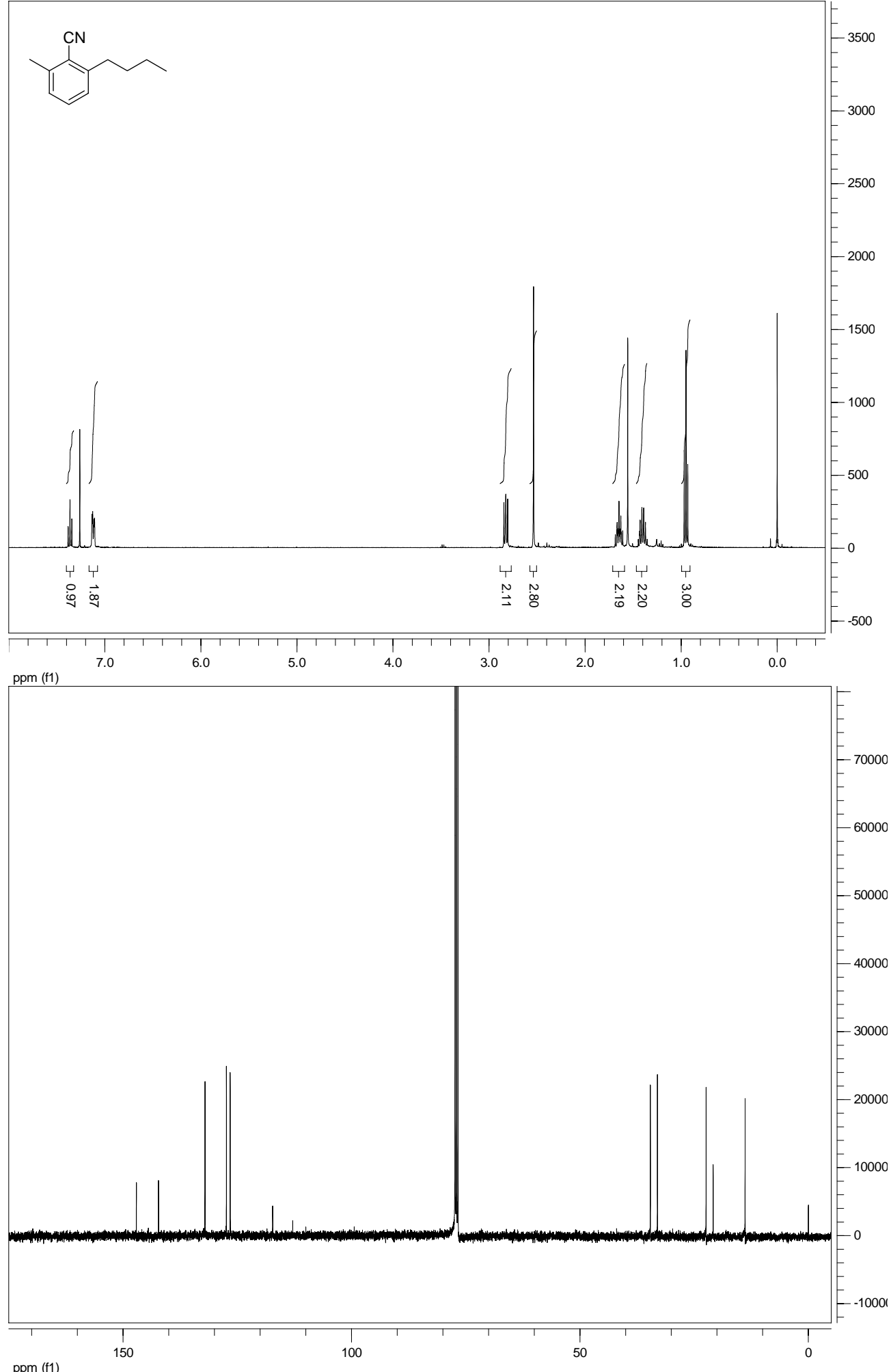


2-Butyl-6-chloro-benzonitrile (27)
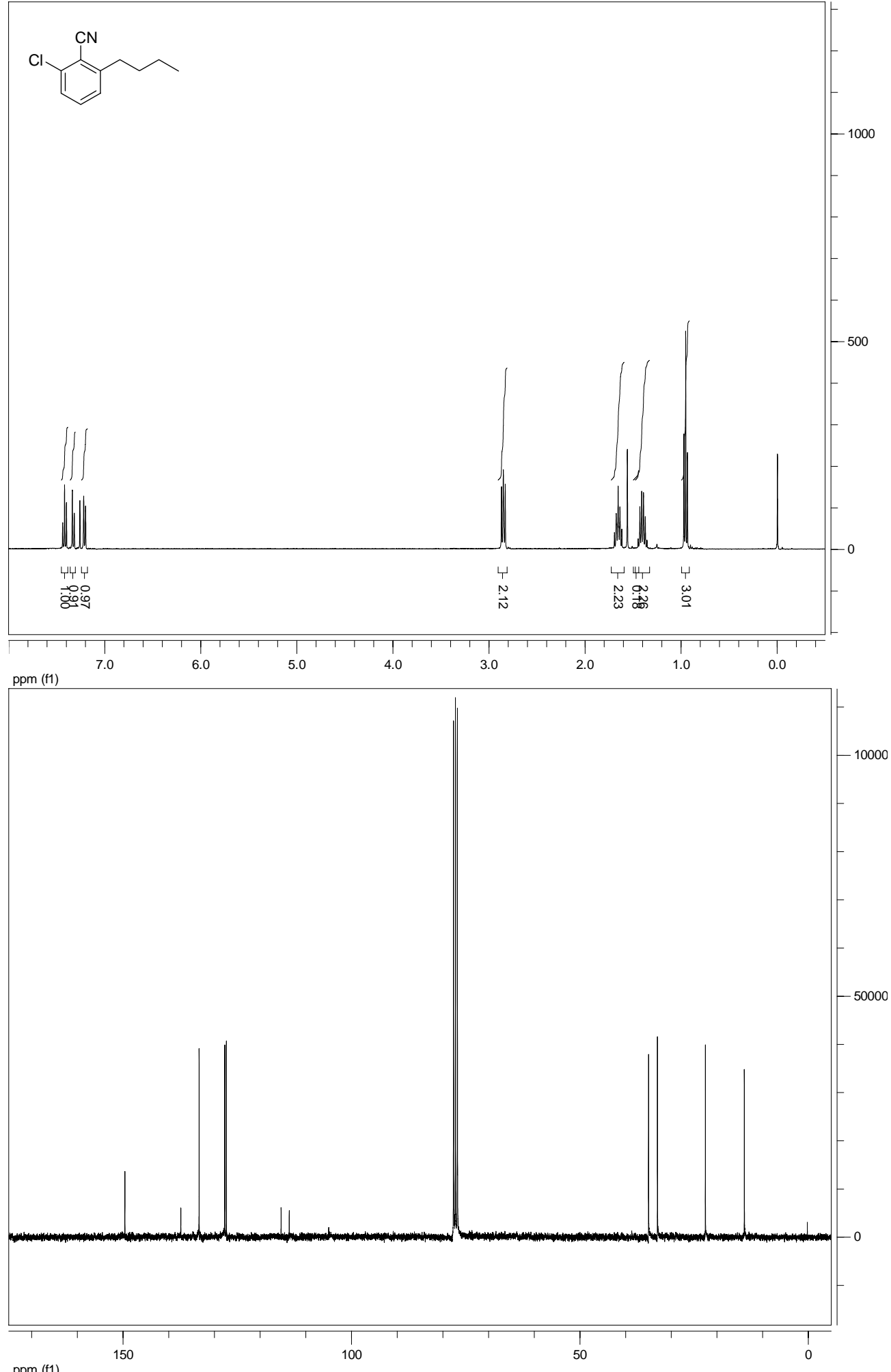
2-Butyl-6-fluoro-benzonitrile (28)
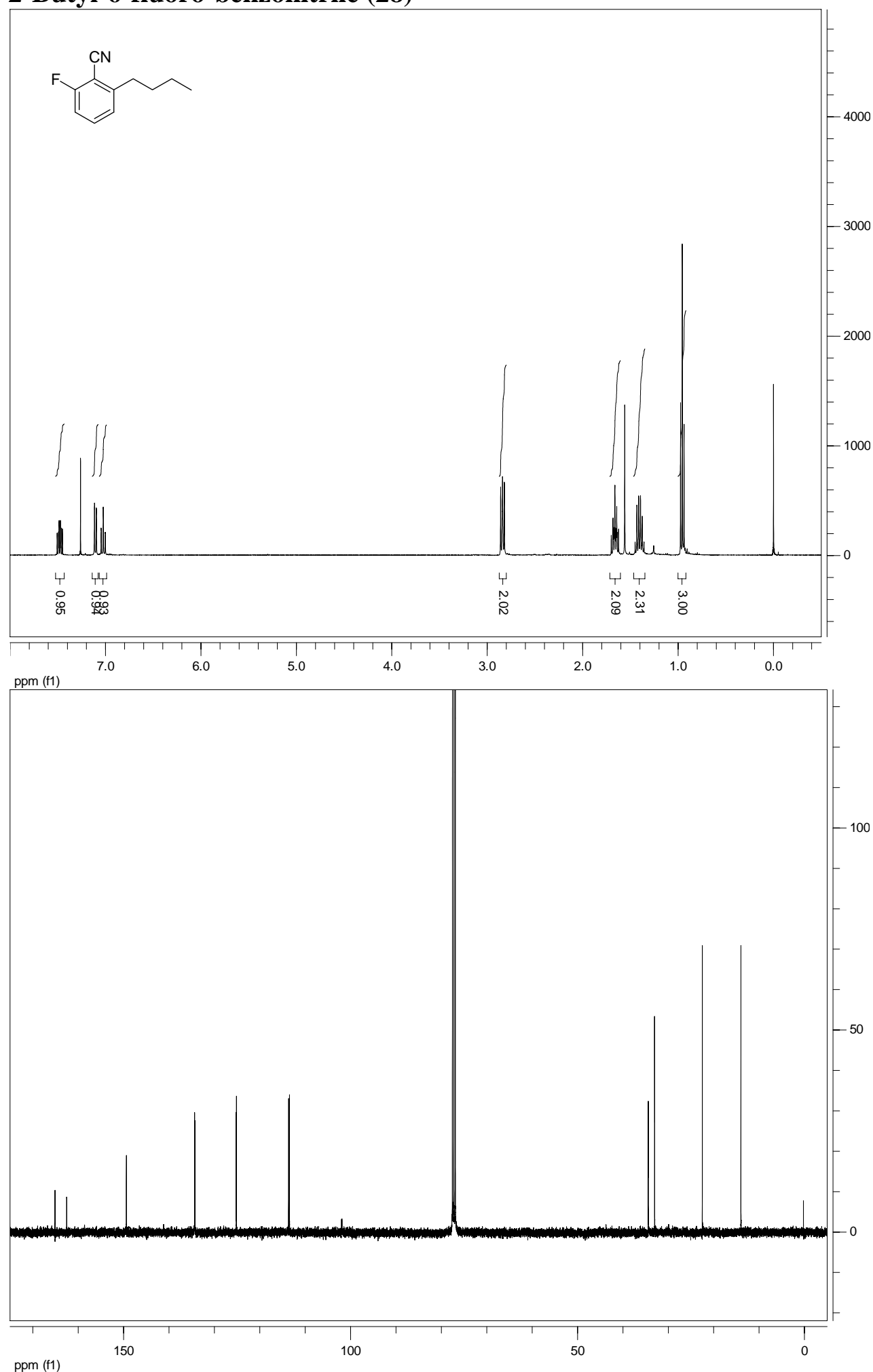


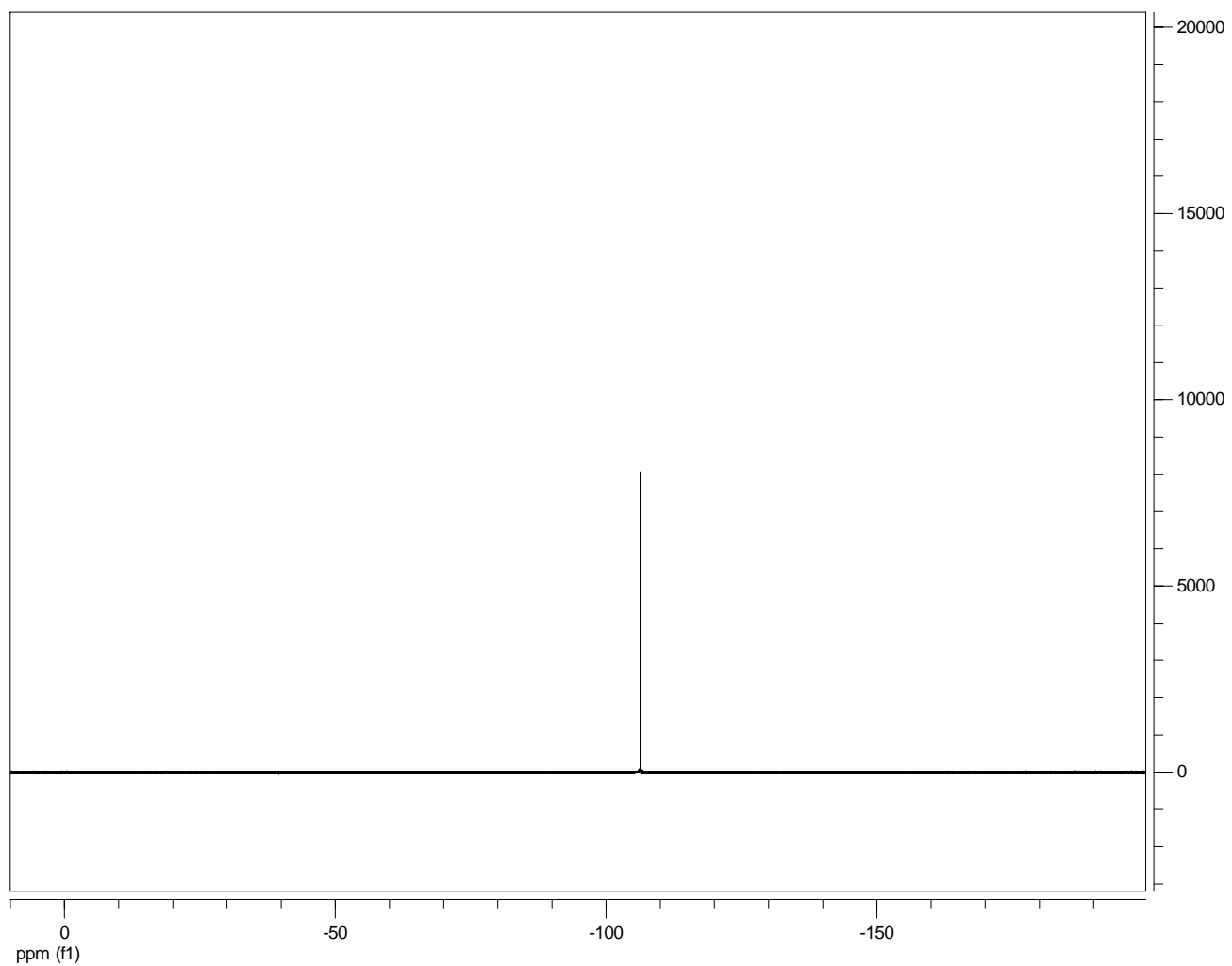


2-Butyl-6-methoxy-benzonitrile (29)
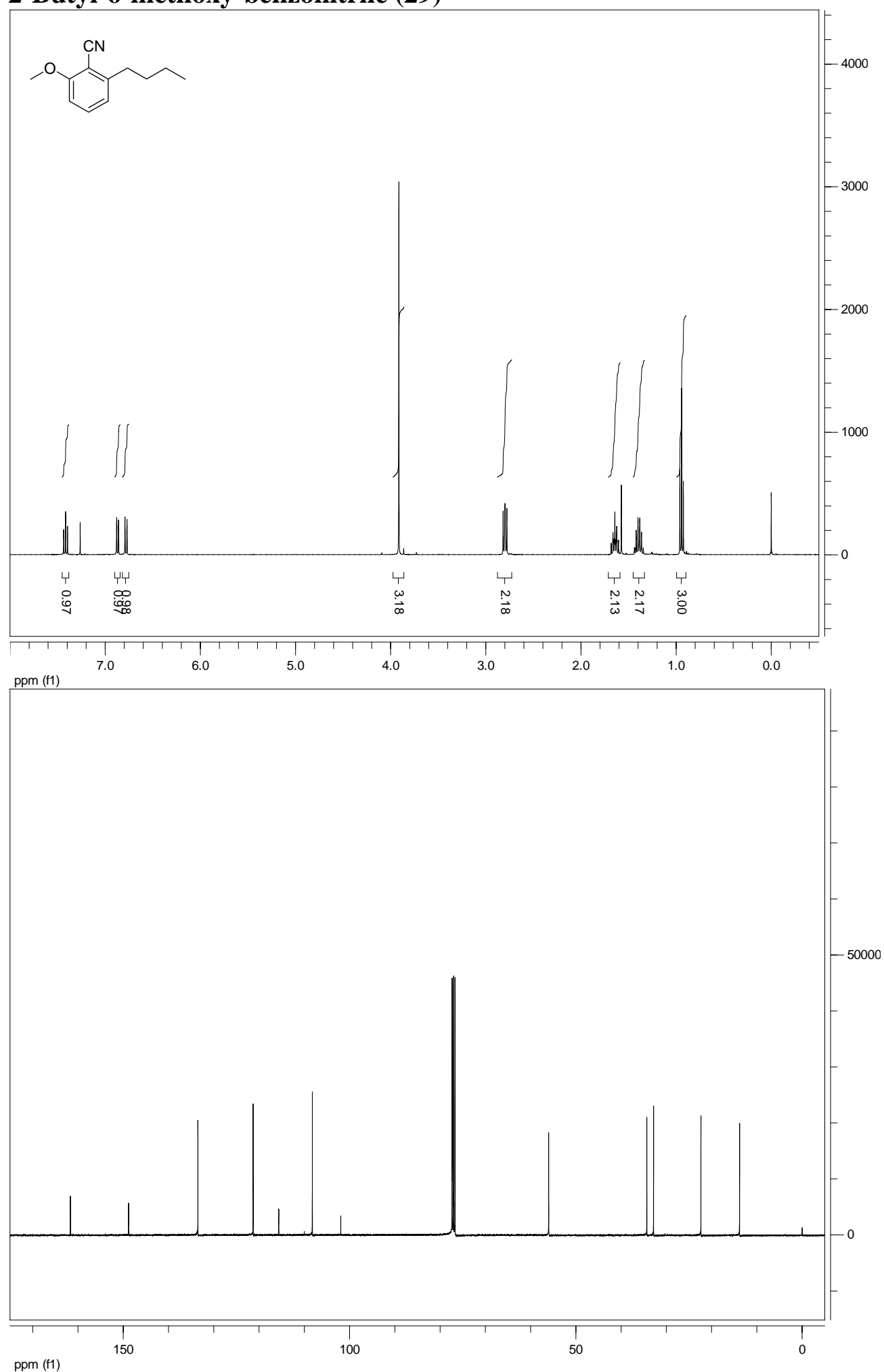


\section{N-(3-Butyl-2-cyano-phenyl)-4,N-dimethyl-benzenesulfonamide (30)}
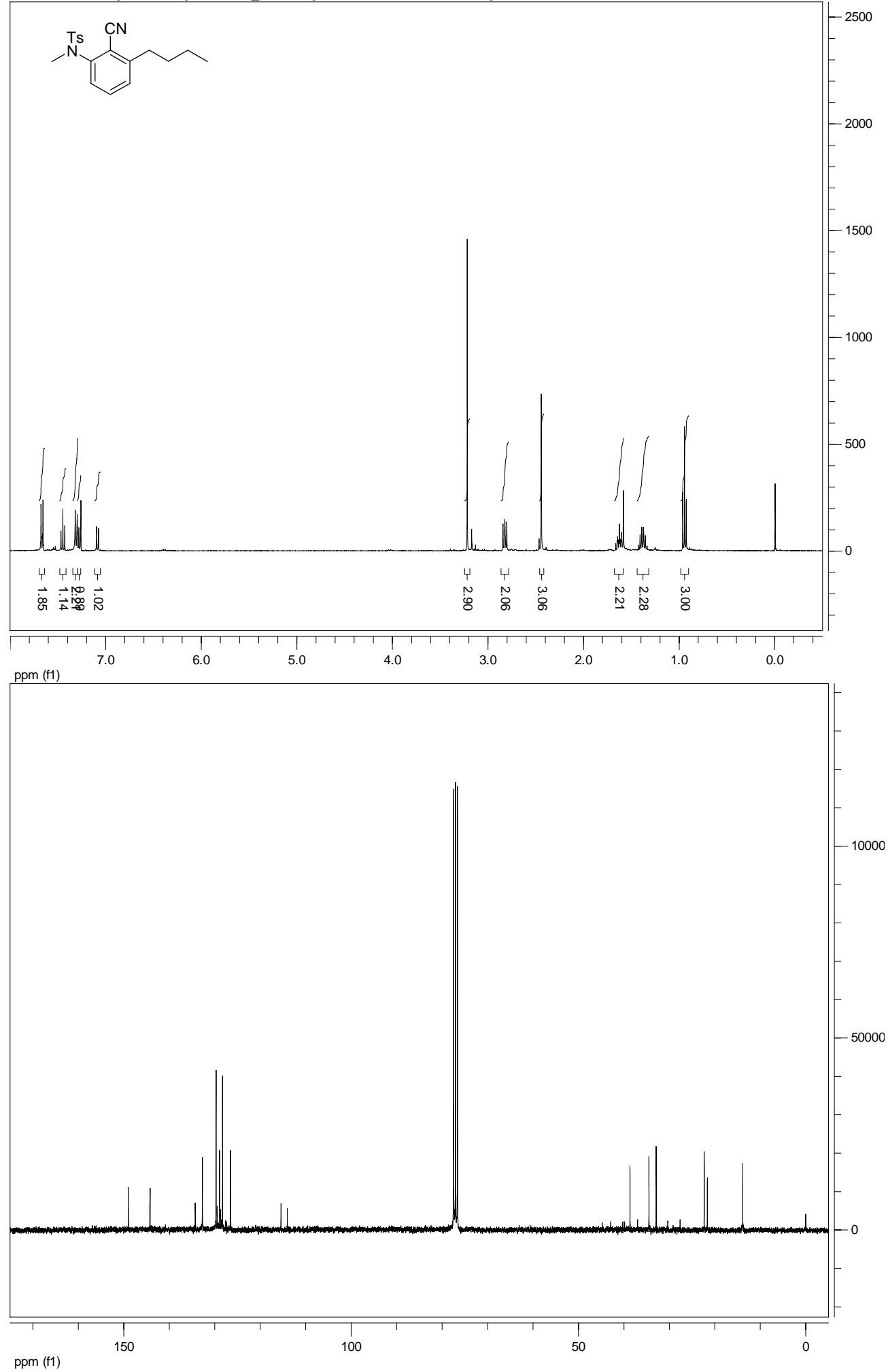
N-(4-Butyl-3-cyano-2-methyl-phenyl)-4,N-dimethyl-benzenesulfonamide (31)

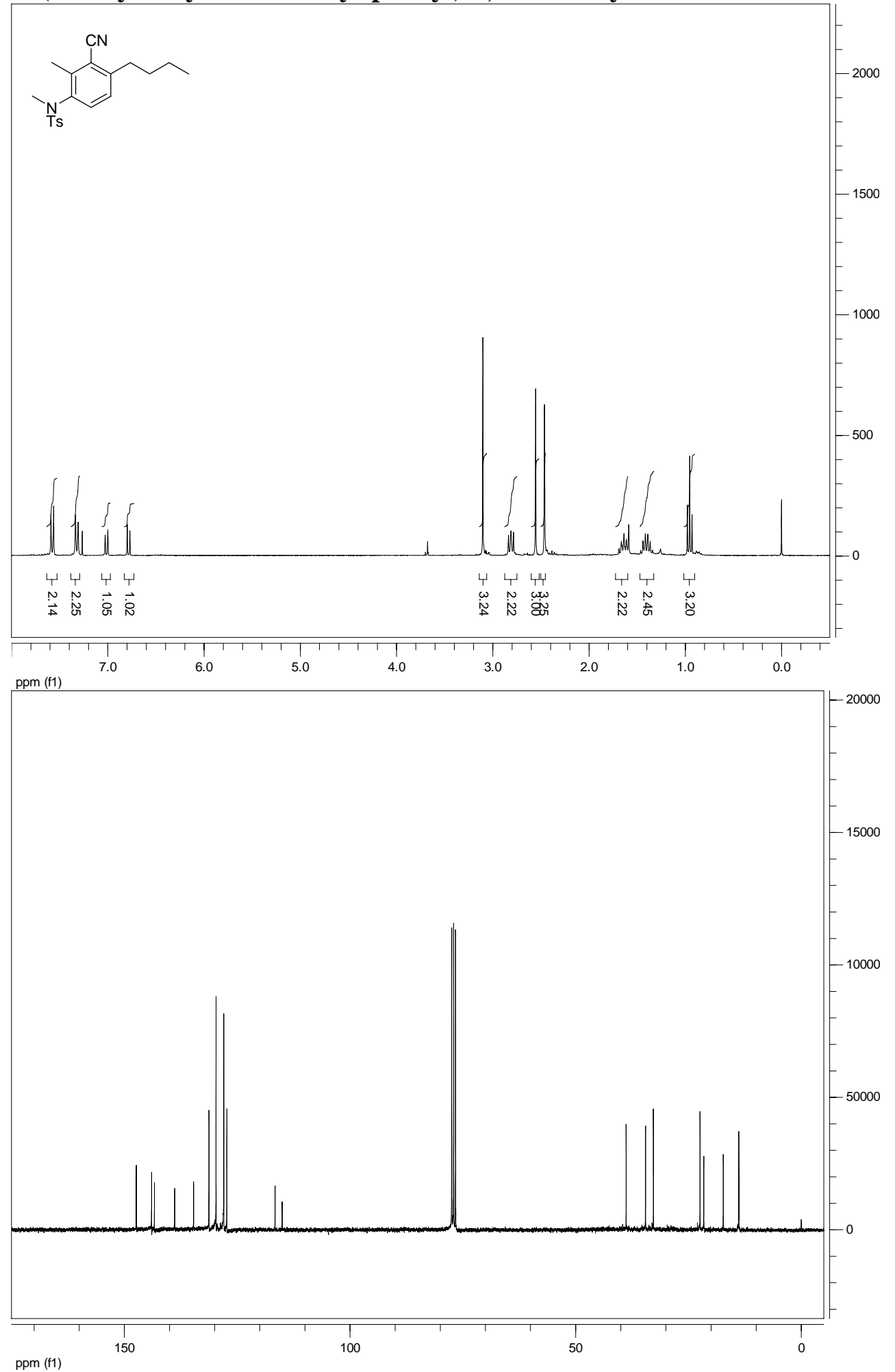




\section{2,6-Dibutyl-4-chloro-benzonitrile (32)}

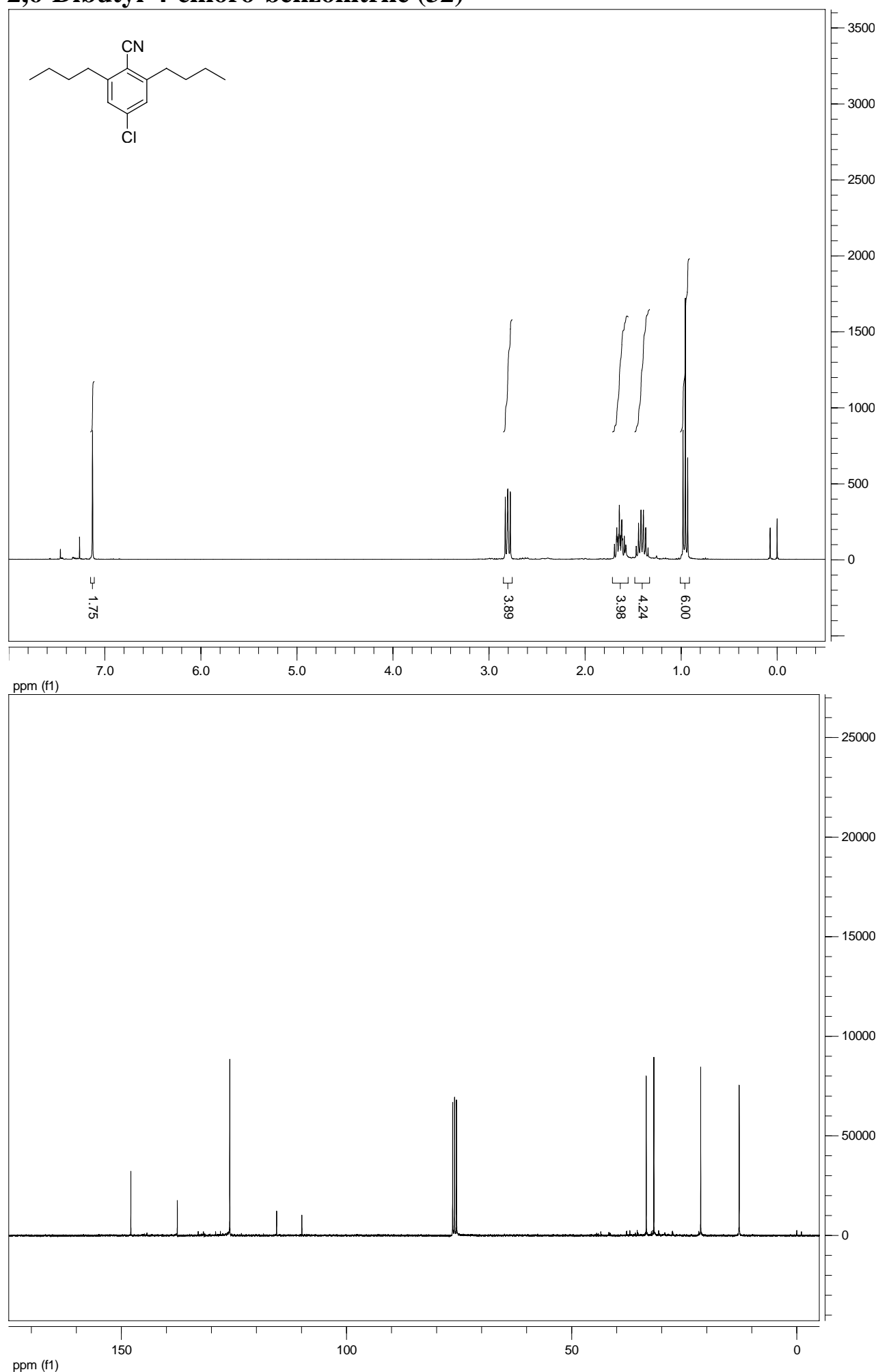


2,6-Dibutyl-4-methoxy-benzonitrile (33)

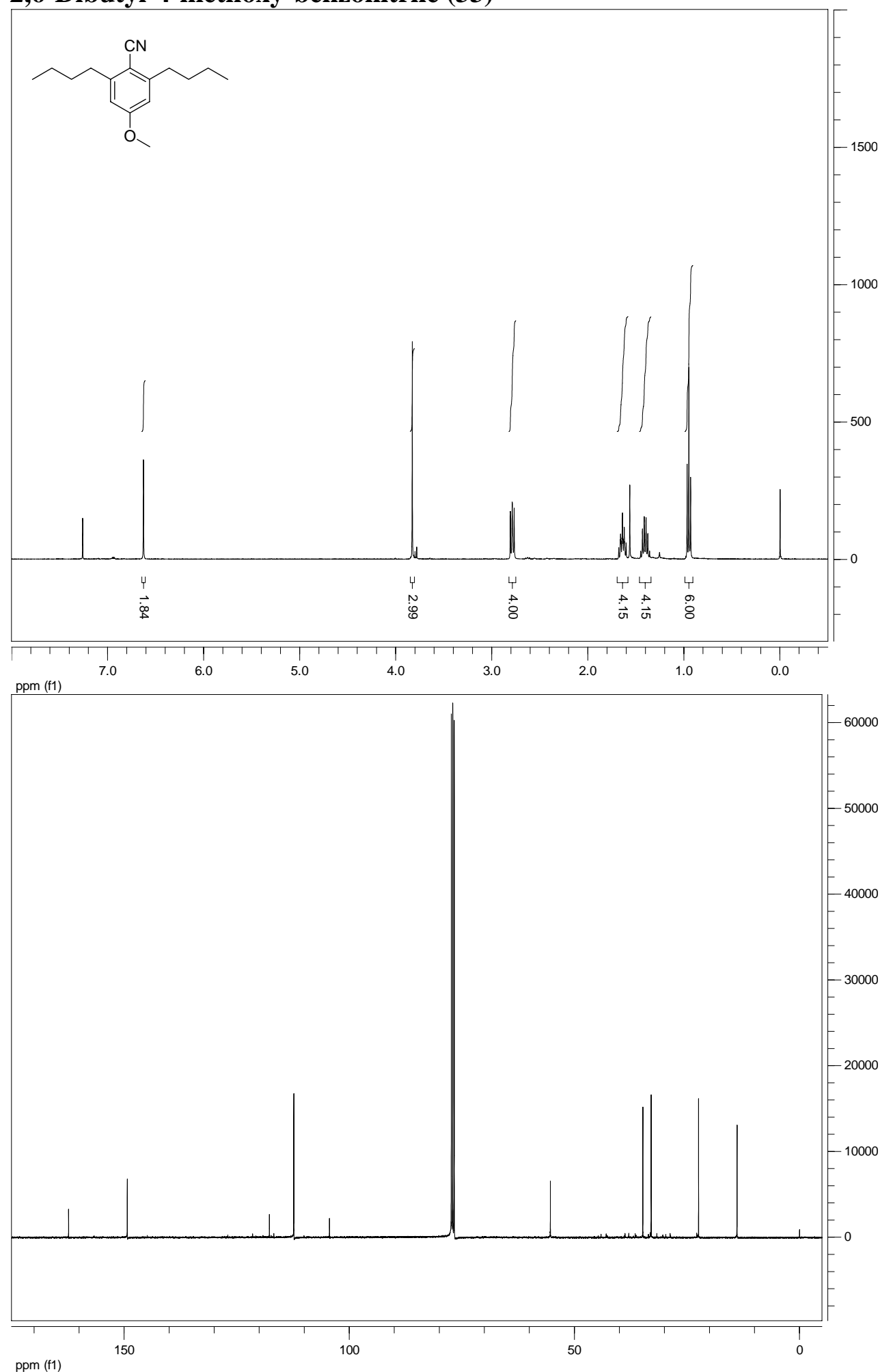


N-(3,5-Dibutyl-4-cyano-phenyl)-4,N-dimethyl-benzenesulfonamide (34)
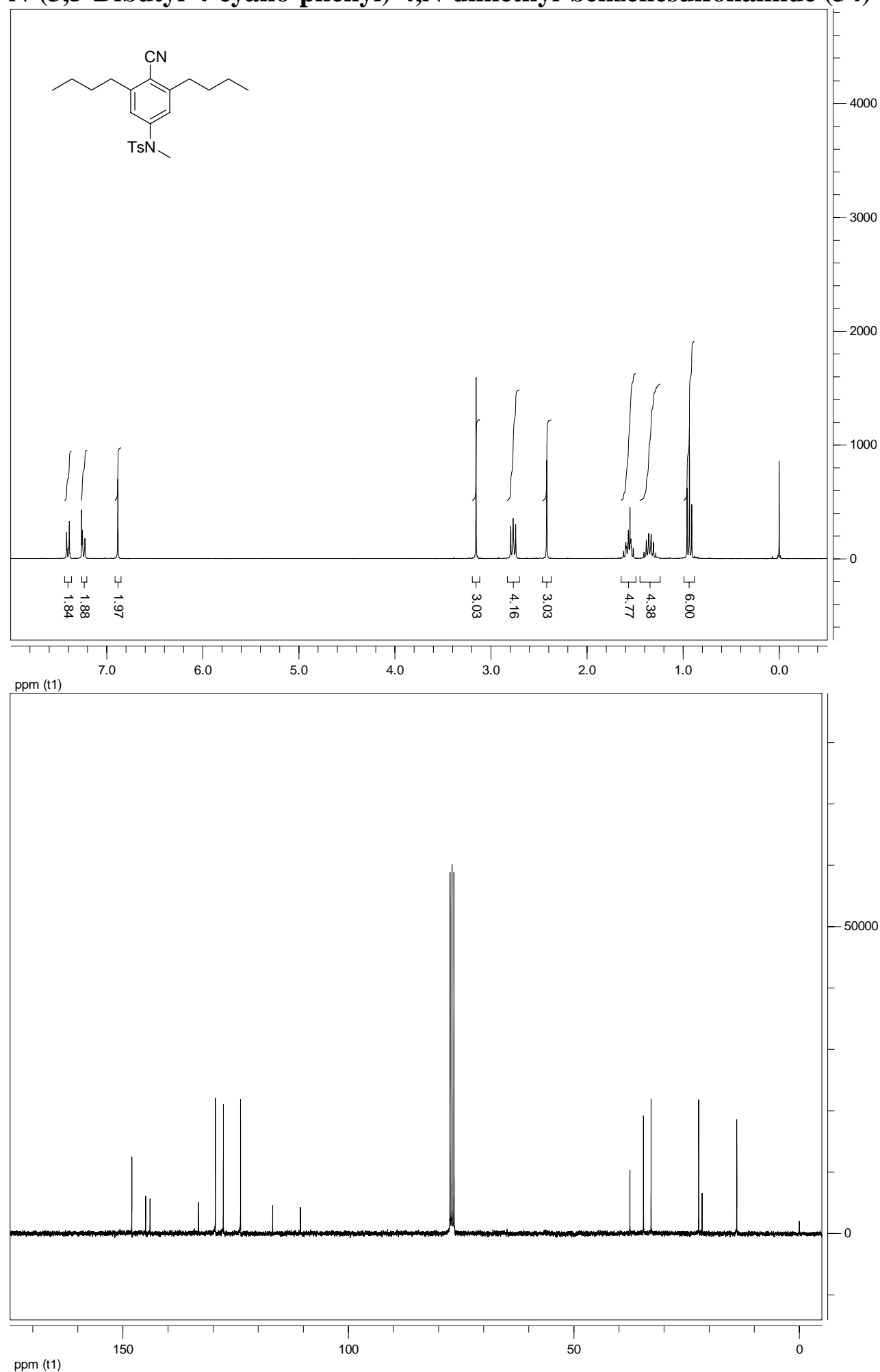


\section{2,6-Dibutyl-4-nitro-benzonitrile (35)}

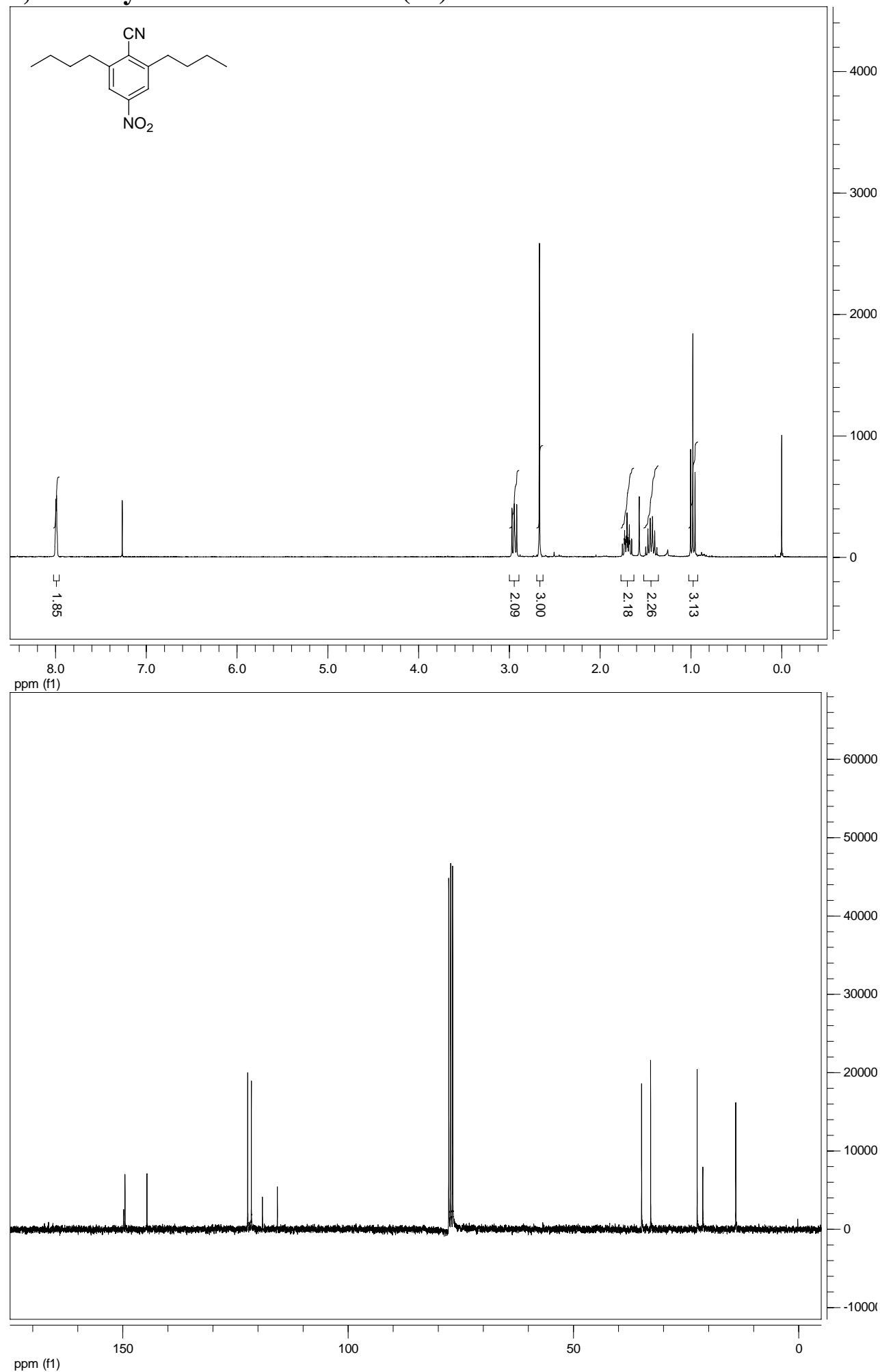




\section{2-Isobutyl-naphthalene-1-carbonitrile (36)}

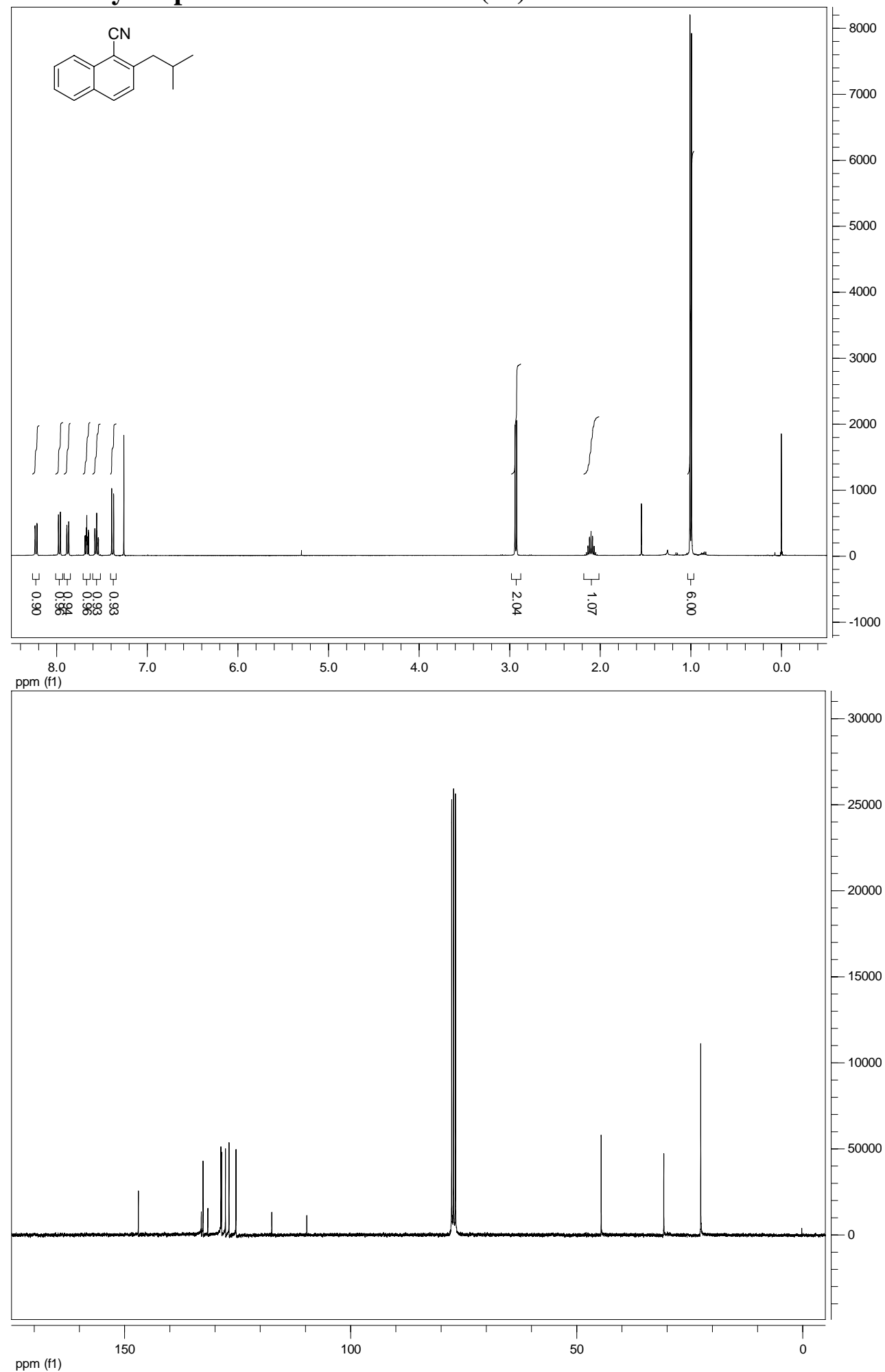


N-(4-Cyano-3,5-dimethyl-phenyl)-4,N-dimethyl-benzenesulfonamide (37)

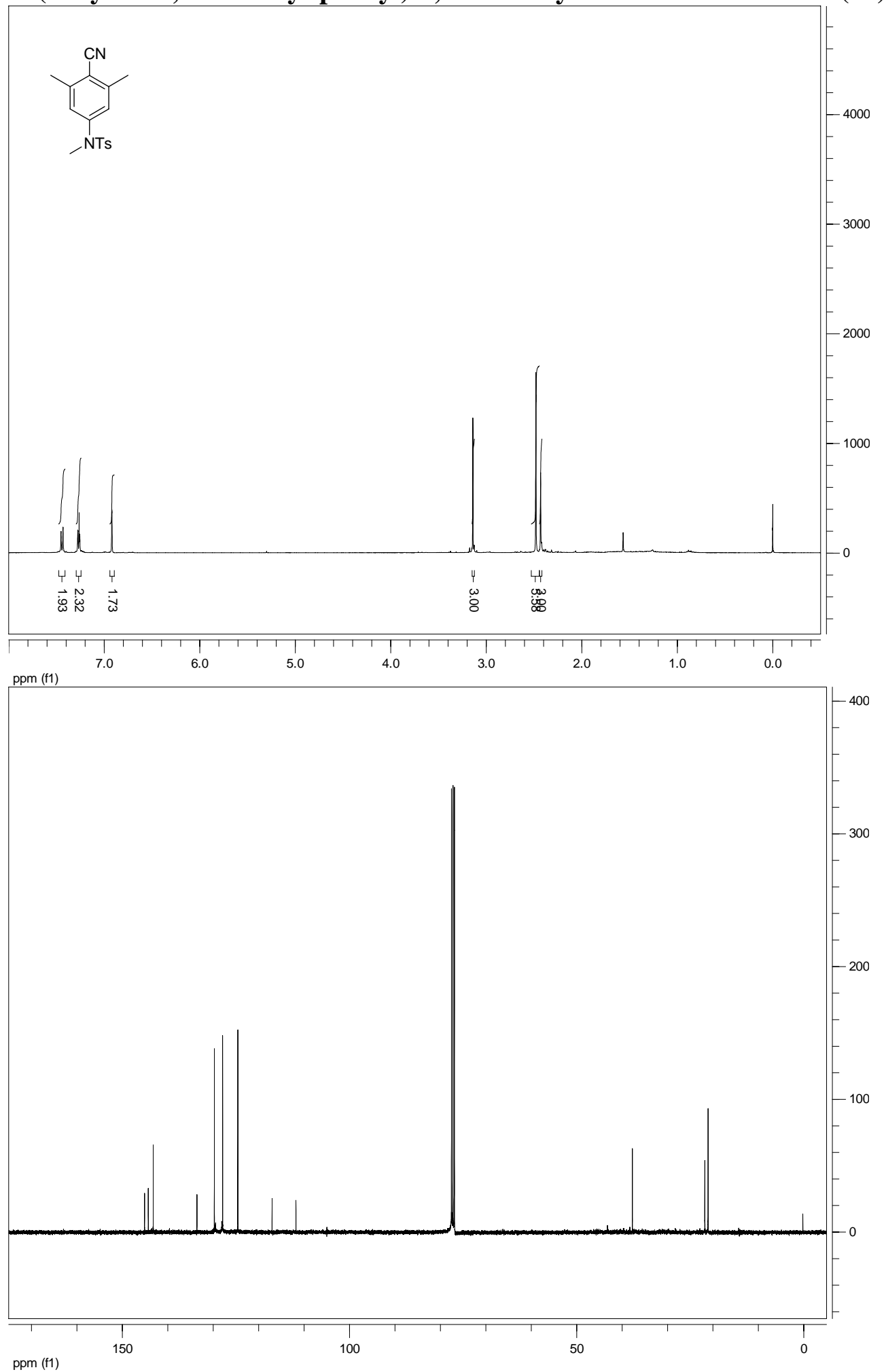


2-(3-Iodo-propyl)-naphthalene-1-carbonitrile (38)

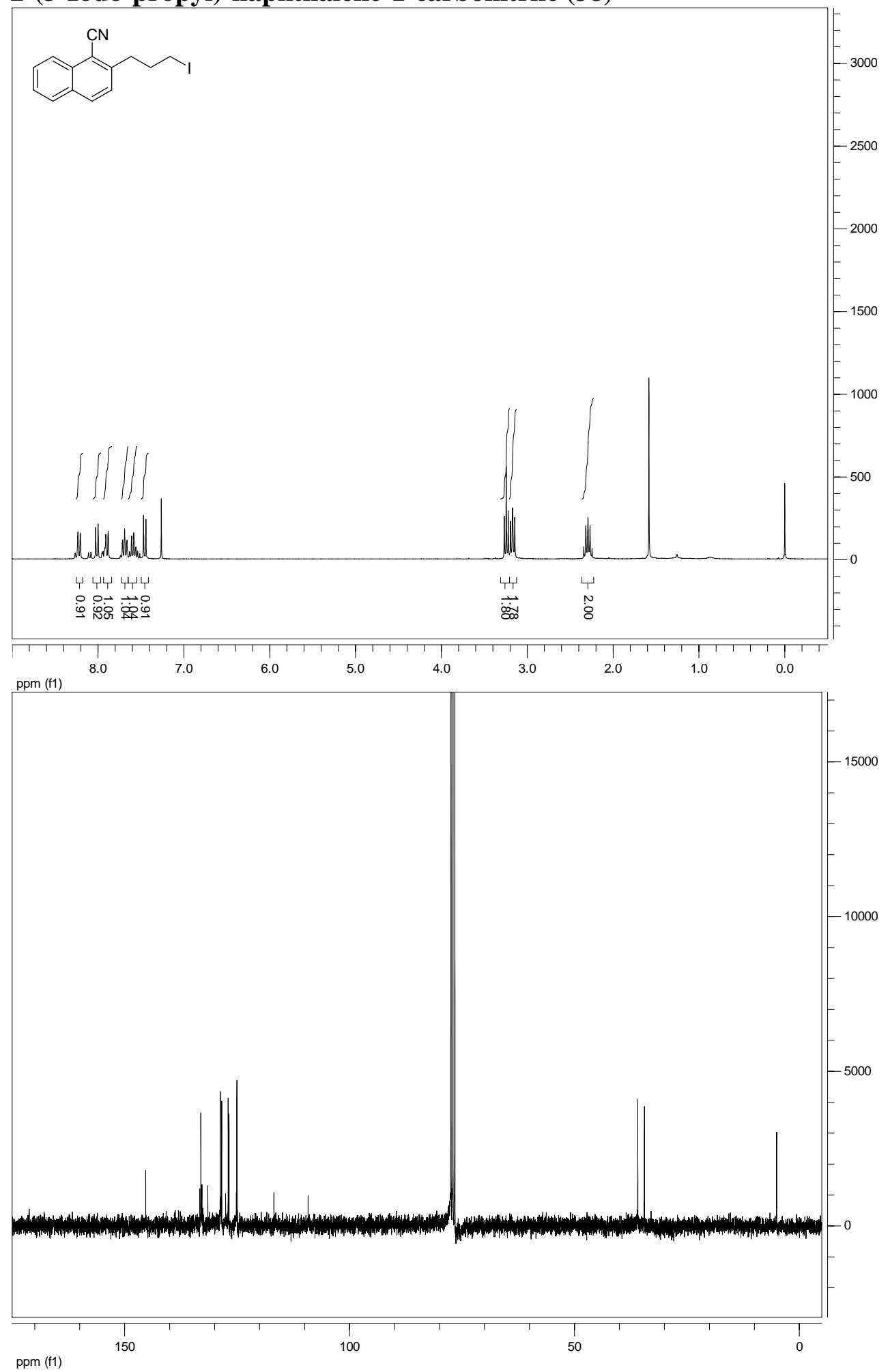


2-(4-Iodo-butyl)-naphthalene-1-carbonitrile (39)

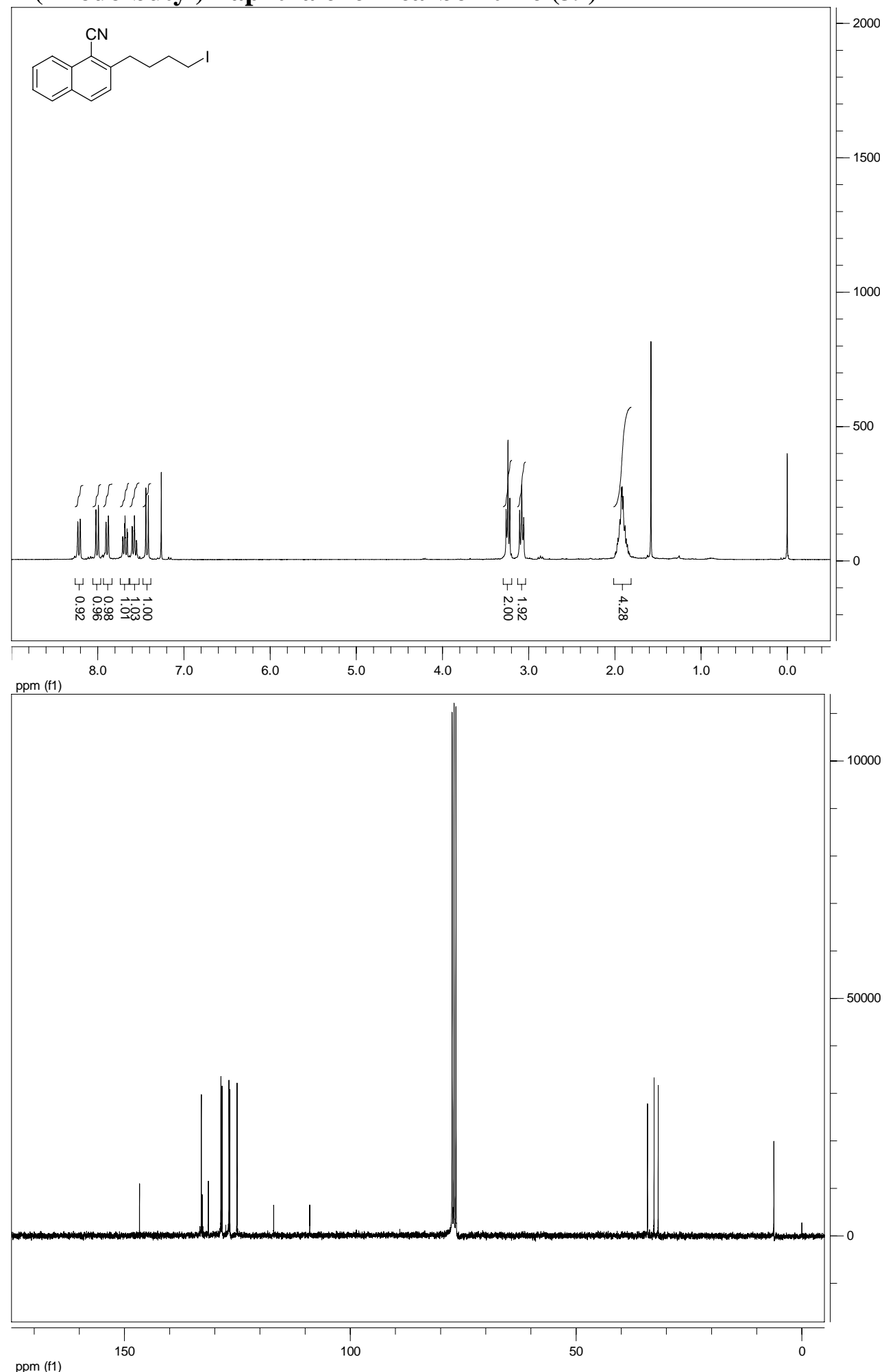


2-(5-Chloro-pentyl)-naphthalene-1-carbonitrile (40)

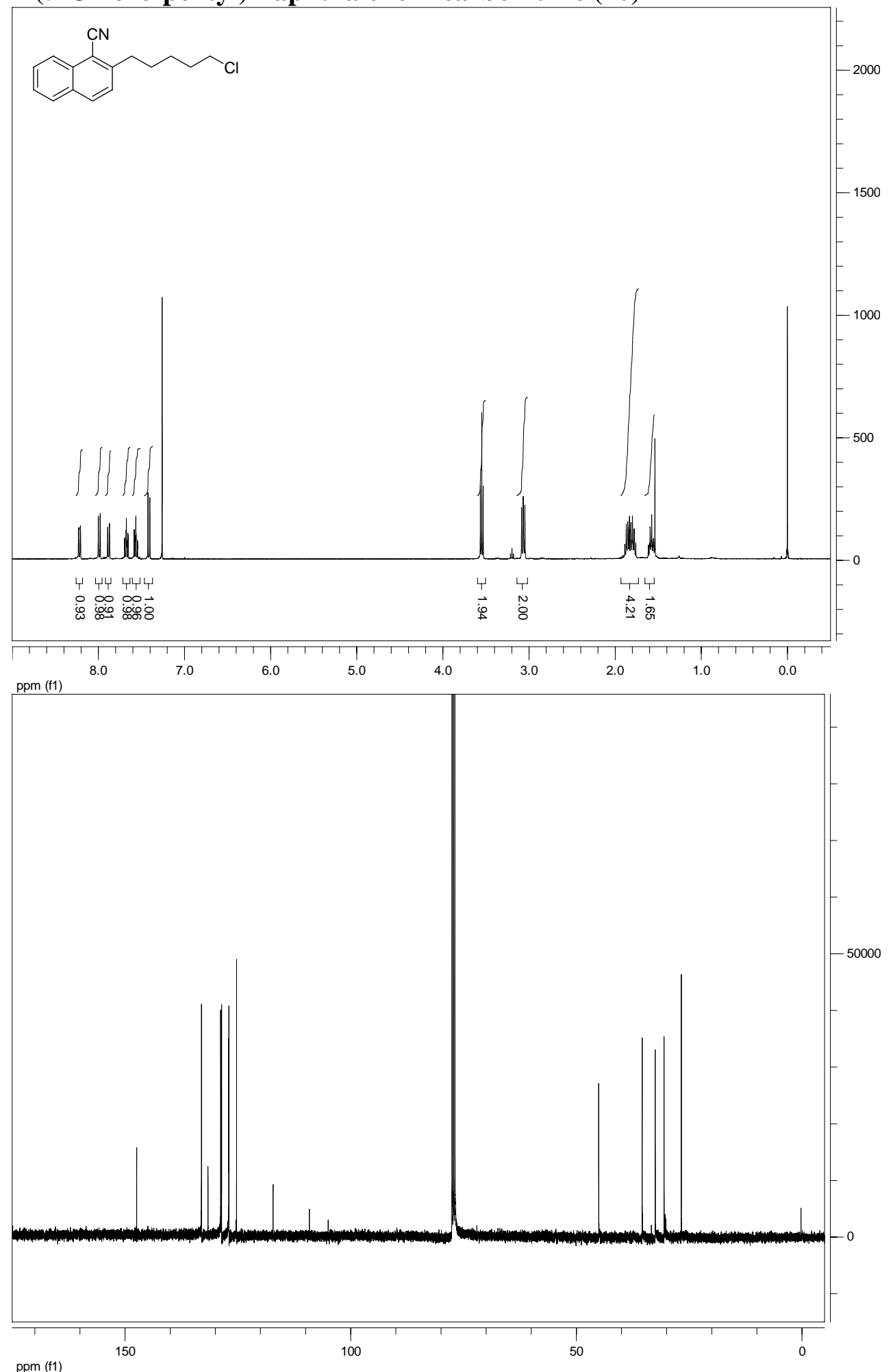




\section{2-(3-Phenyl-propyl)-naphthalene-1-carbonitrile (41)}

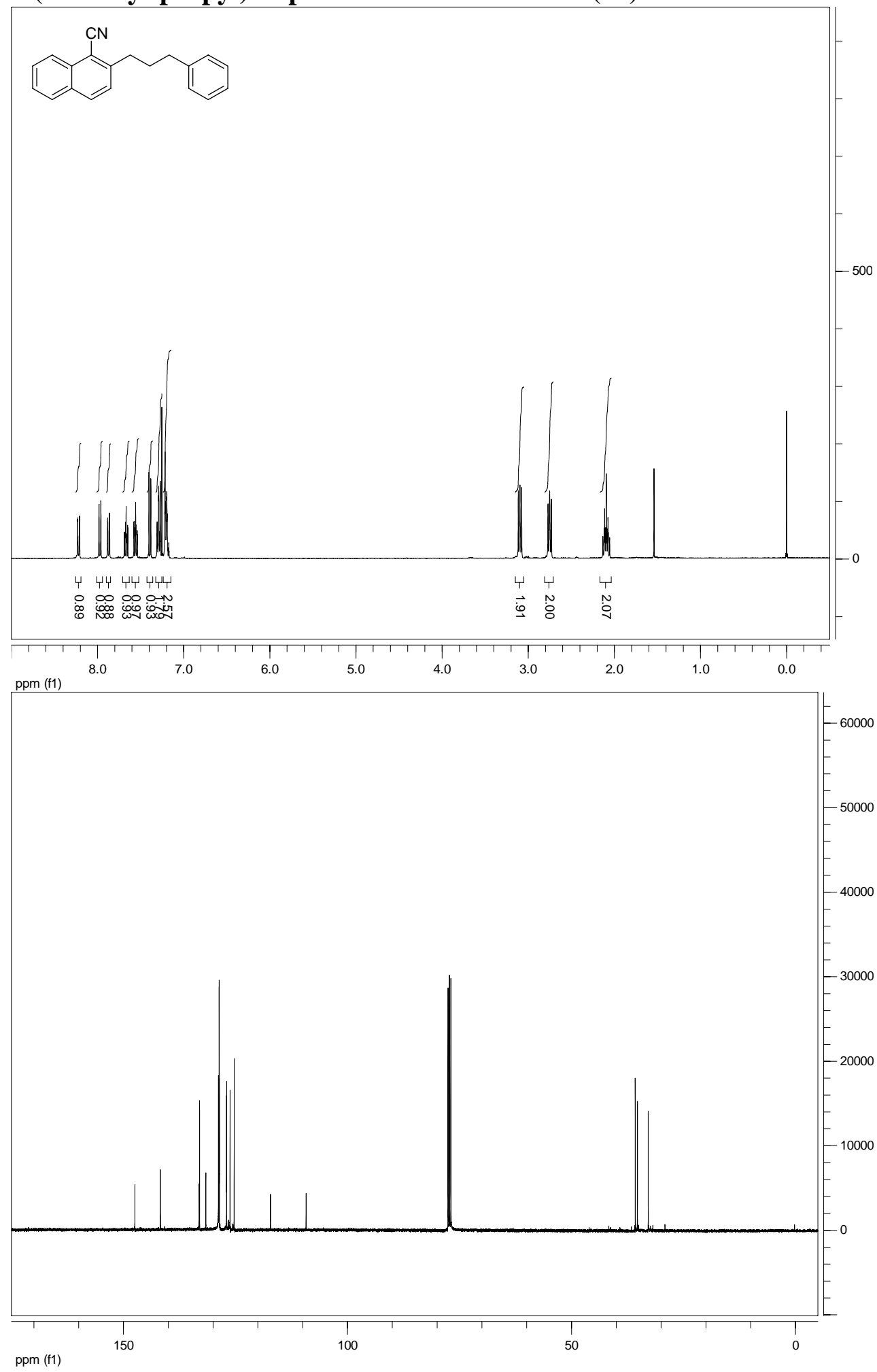


2-(3,3-Diethoxy-propyl)-naphthalene-1-carbonitrile (43)
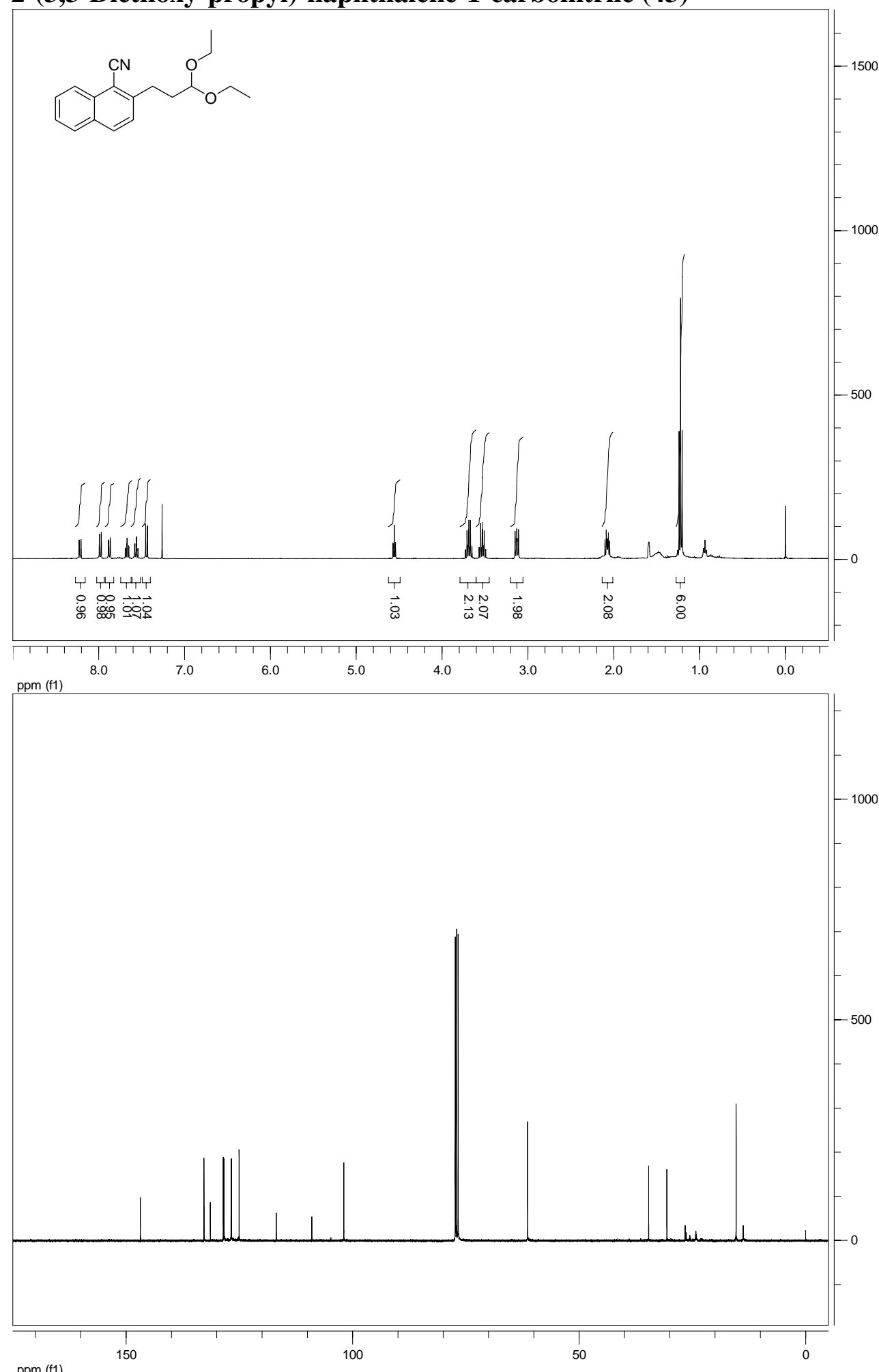

ppm (f1) 
2-[2-(tert-butyl-dimethyl-silanyloxy)-pentyl]-naphtalene-1-carbonitrile (44)

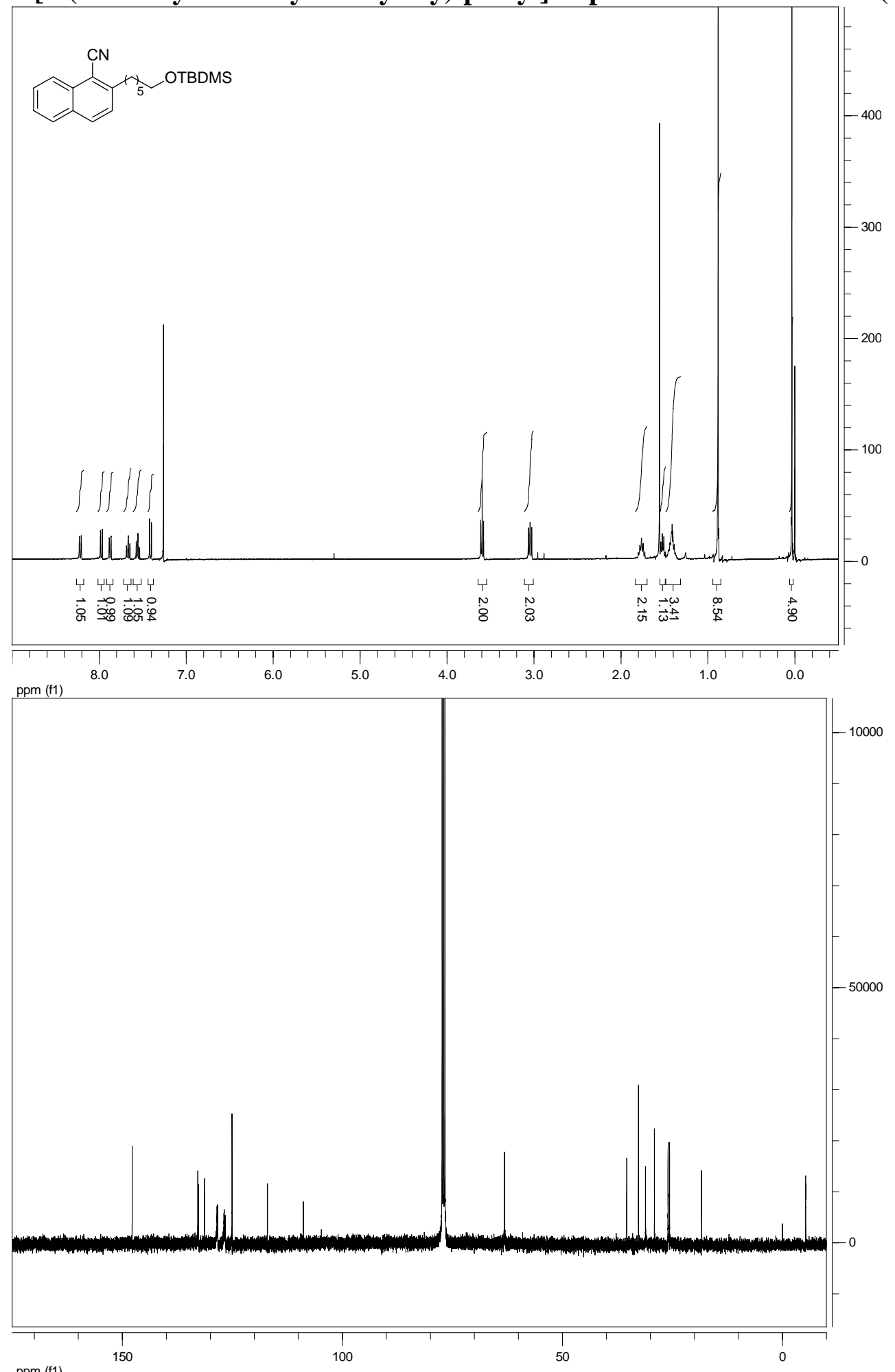


(1-Cyano-naphthalen-2-yl)-acetic acid tert-butyl ester (45)
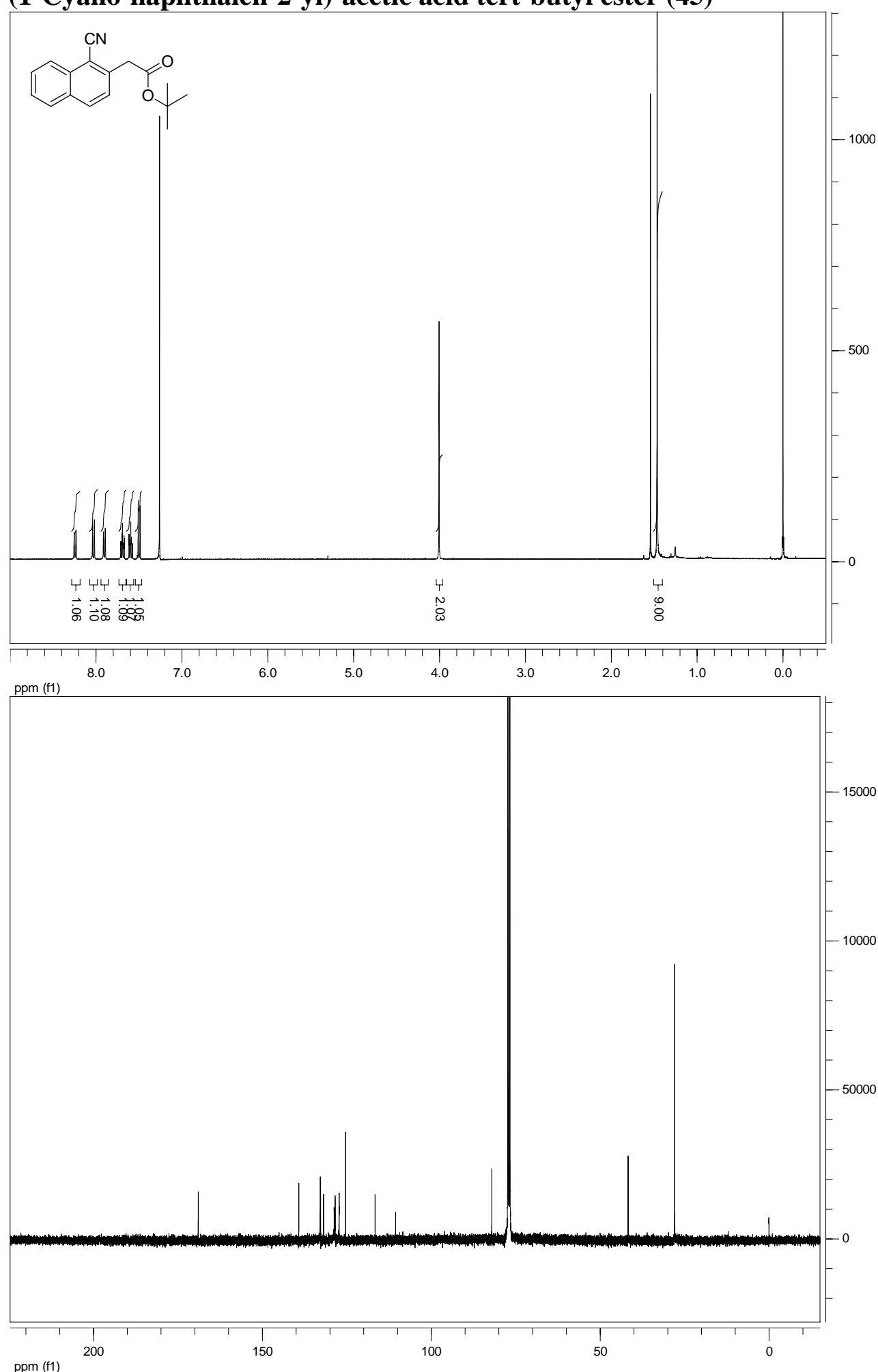
2-(1-Cyano-naphthalen-2-yl)-N,N-diethyl-acetamide (46)

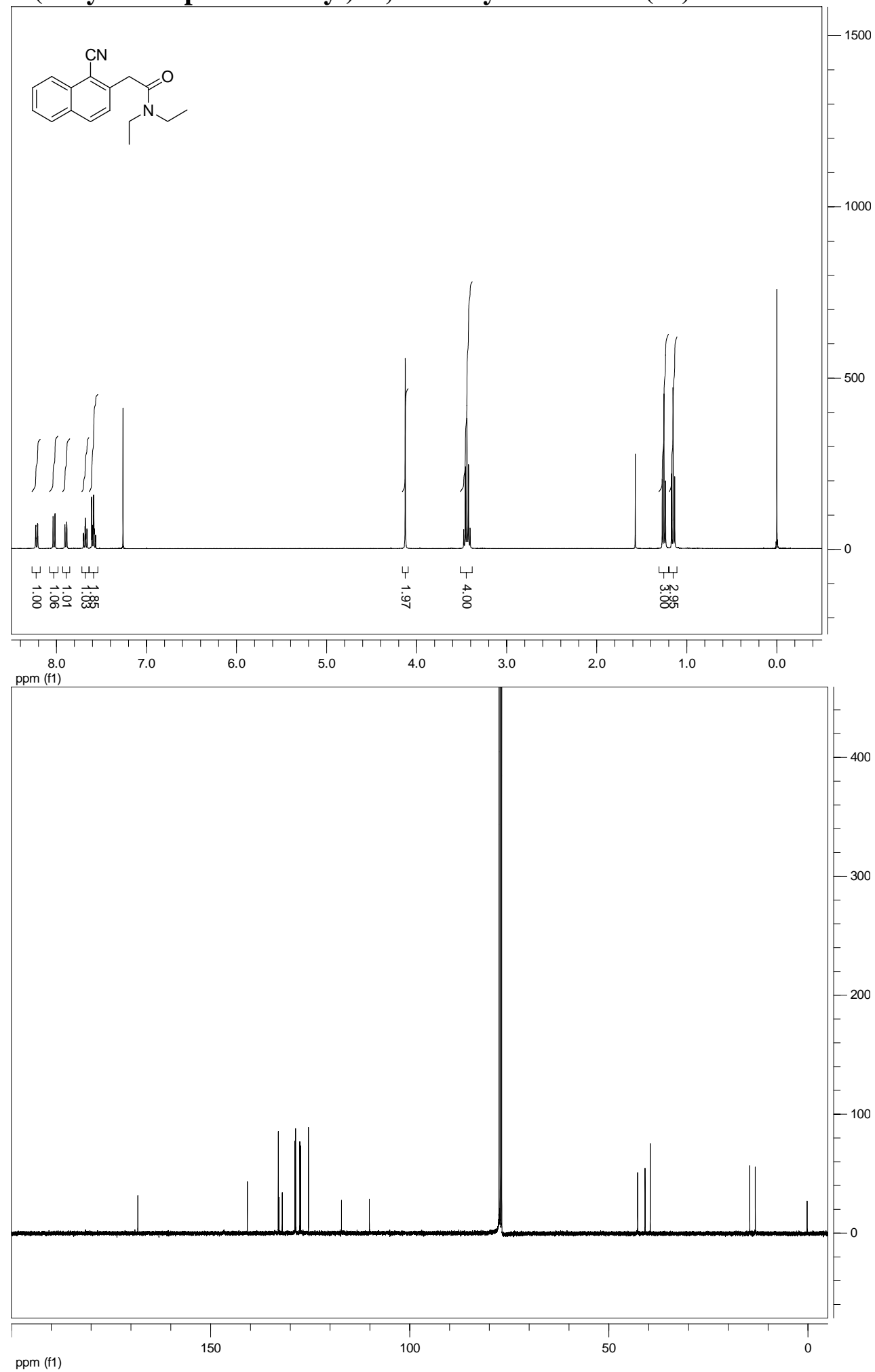


2-Benzyl-naphthalene-1-carbonitrile (47)
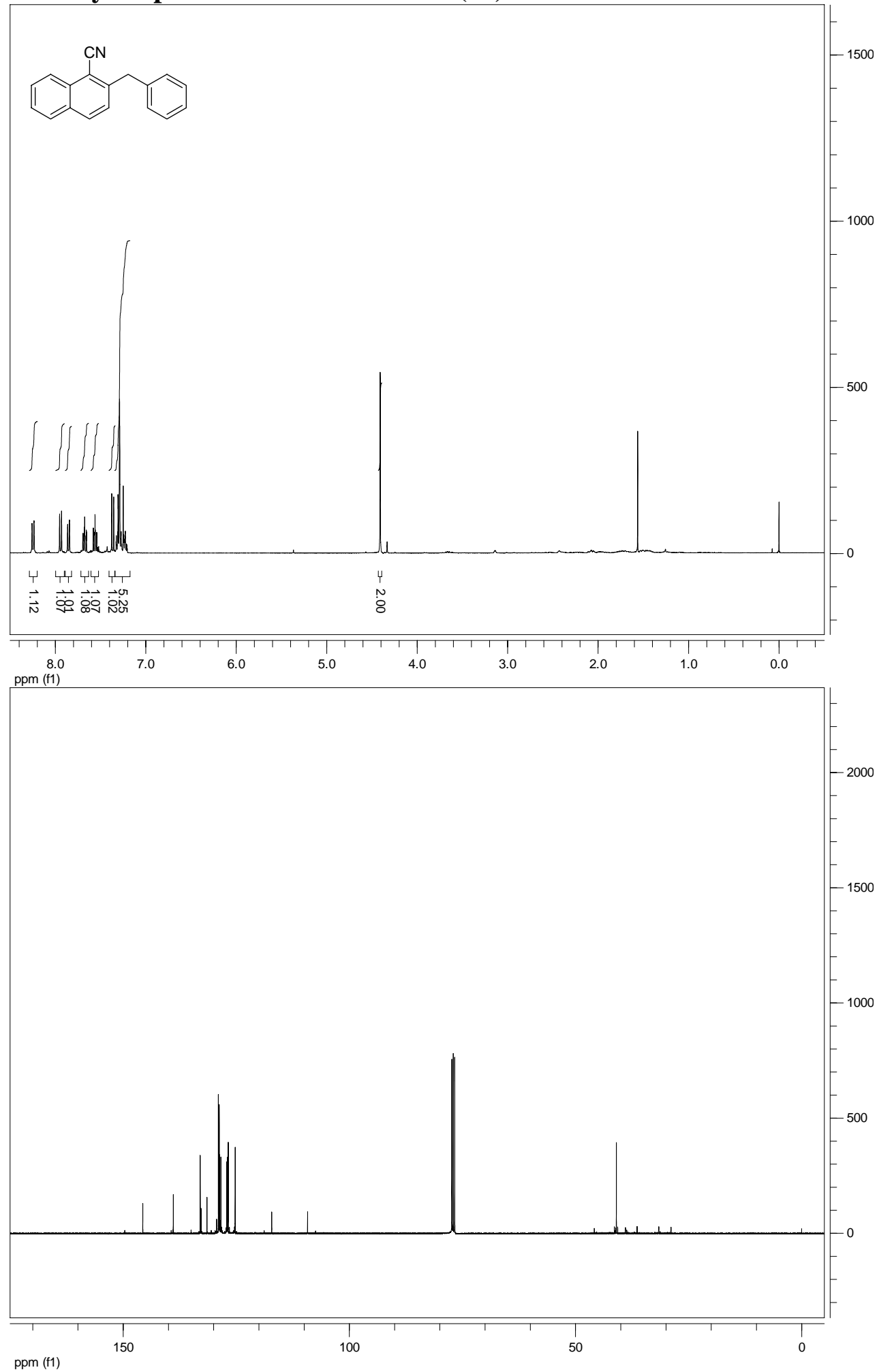
2-(4-Methoxy-benzyl)-naphthalene-1-carbonitrile (48)
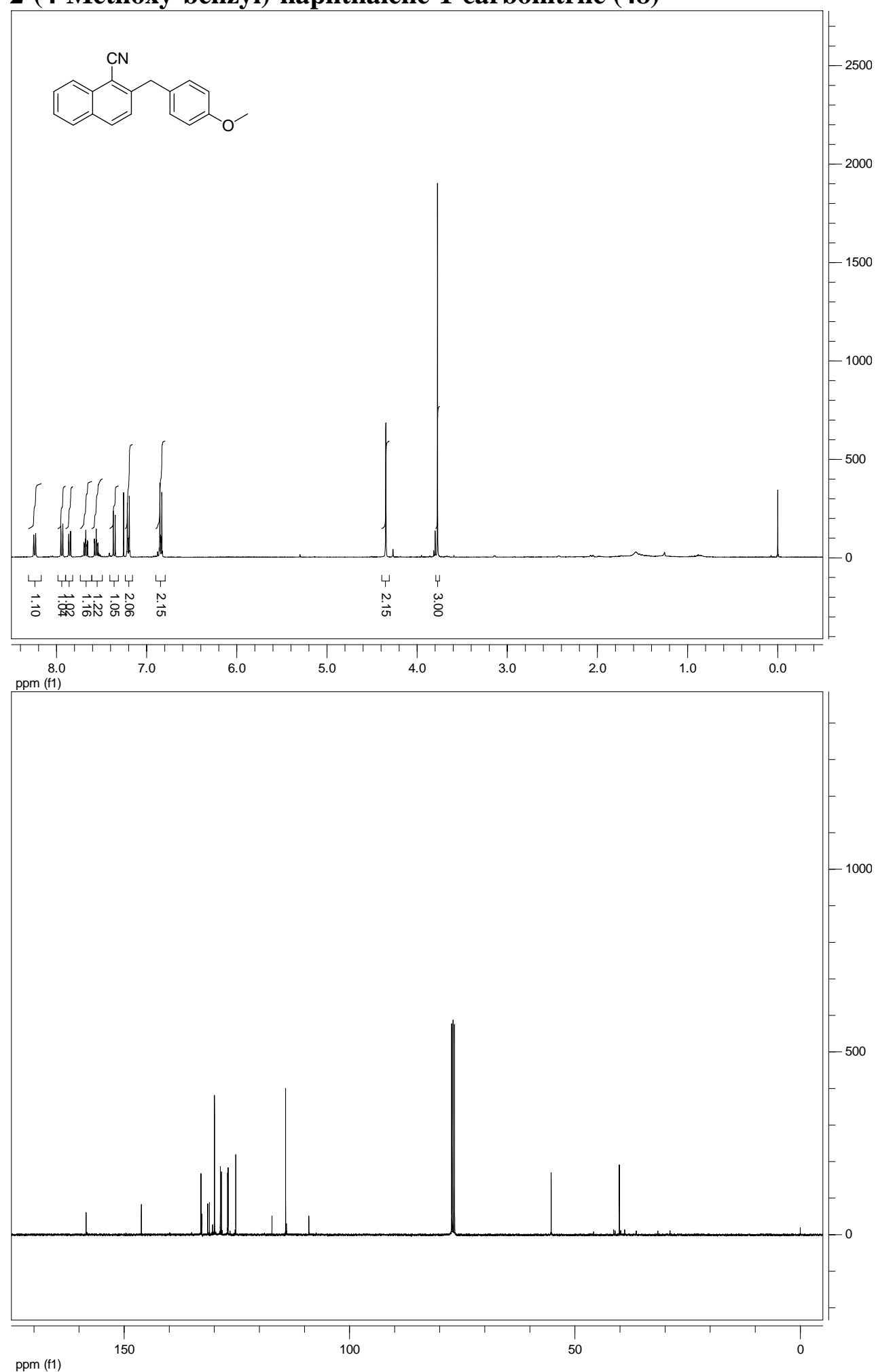
2-(4-Nitro-benzyl)-naphthalene-1-carbonitrile (49)
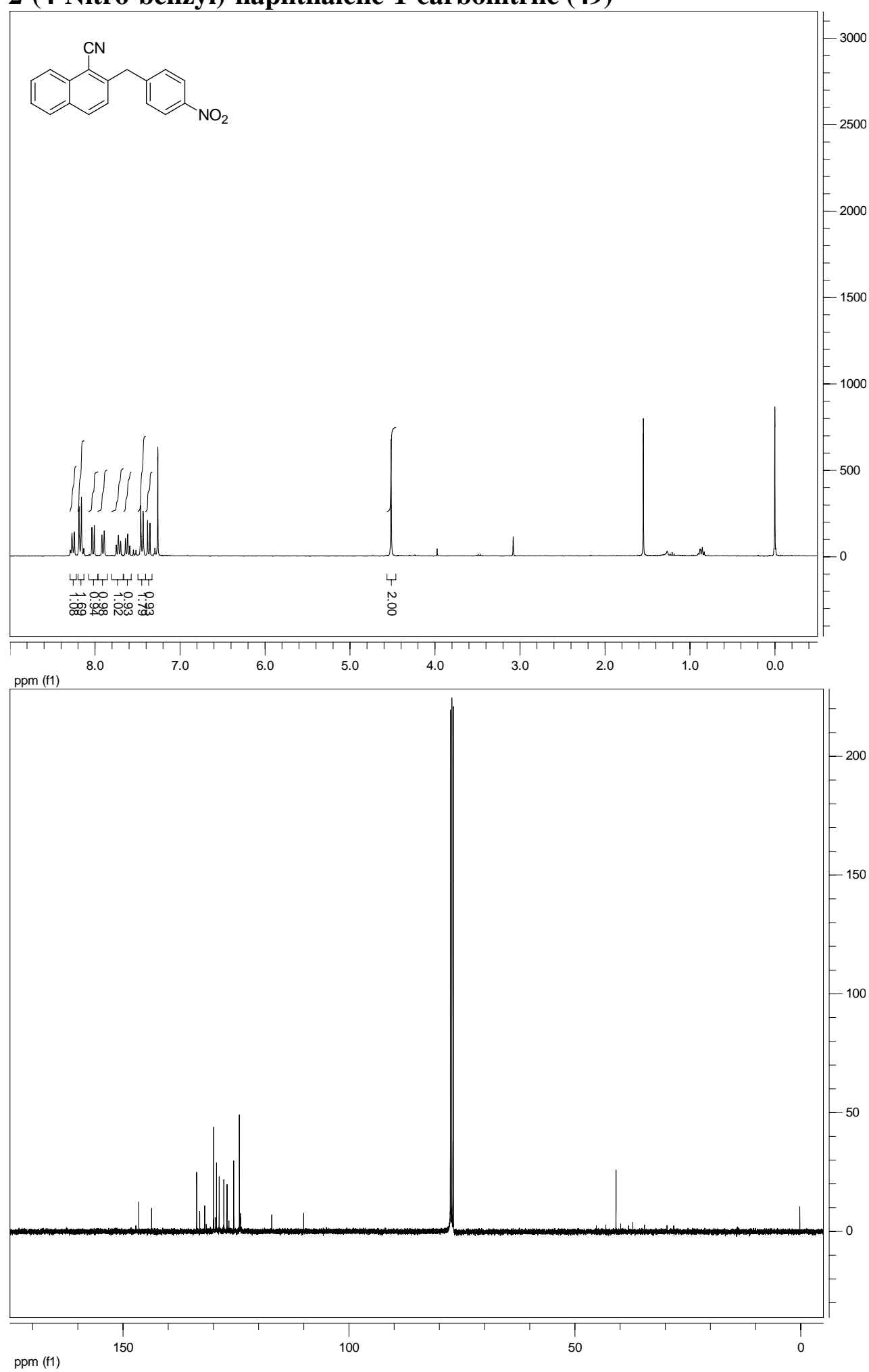
2-Methyl-6-(3-phenyl-propyl)-benzonitrile (50)
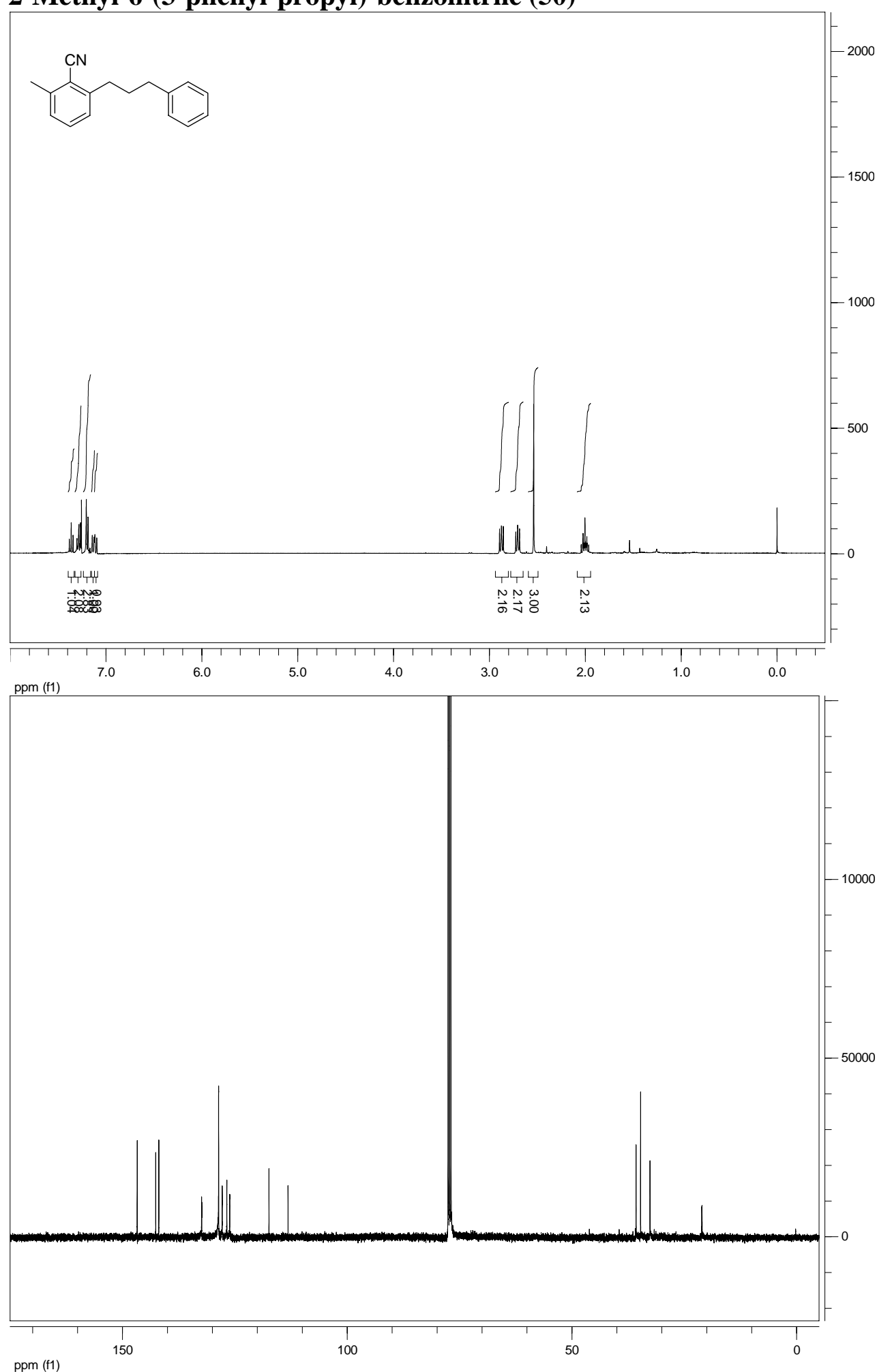


\section{2-Chloro-6-(3-phenyl-propyl)-benzonitrile (51)}
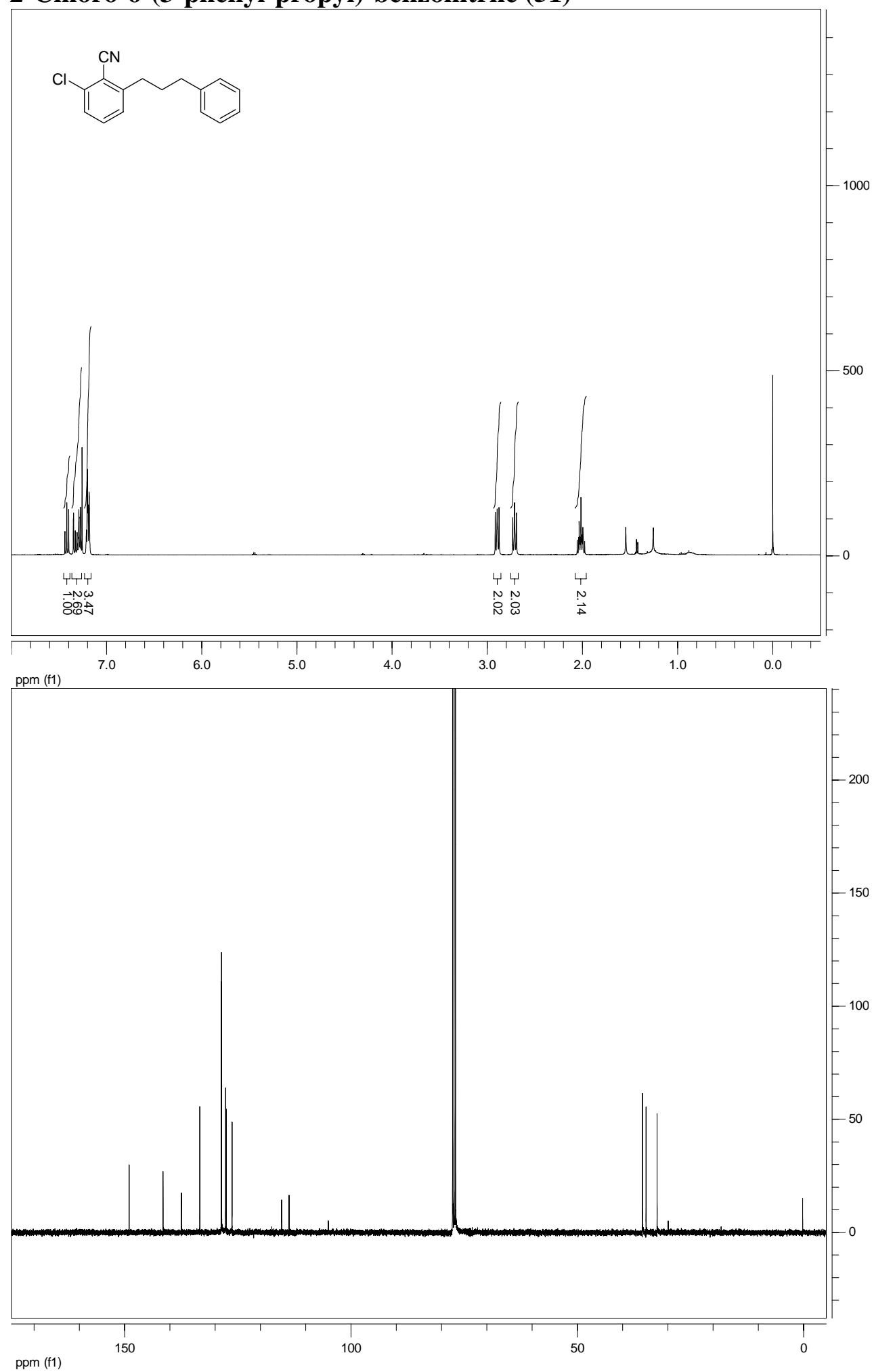
2-Fluoro-6-(3-phenyl-propyl)-benzonitrile (52)
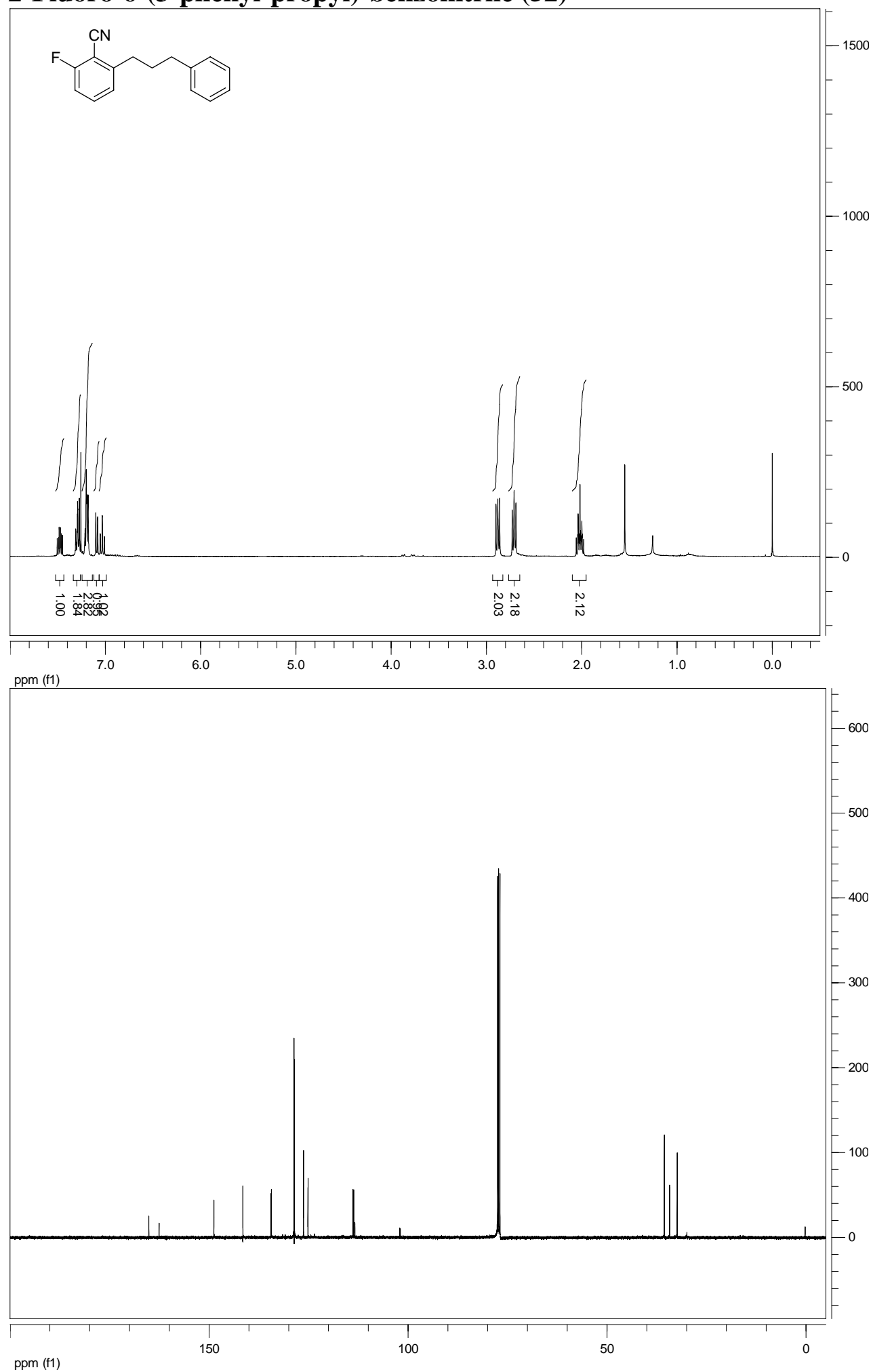


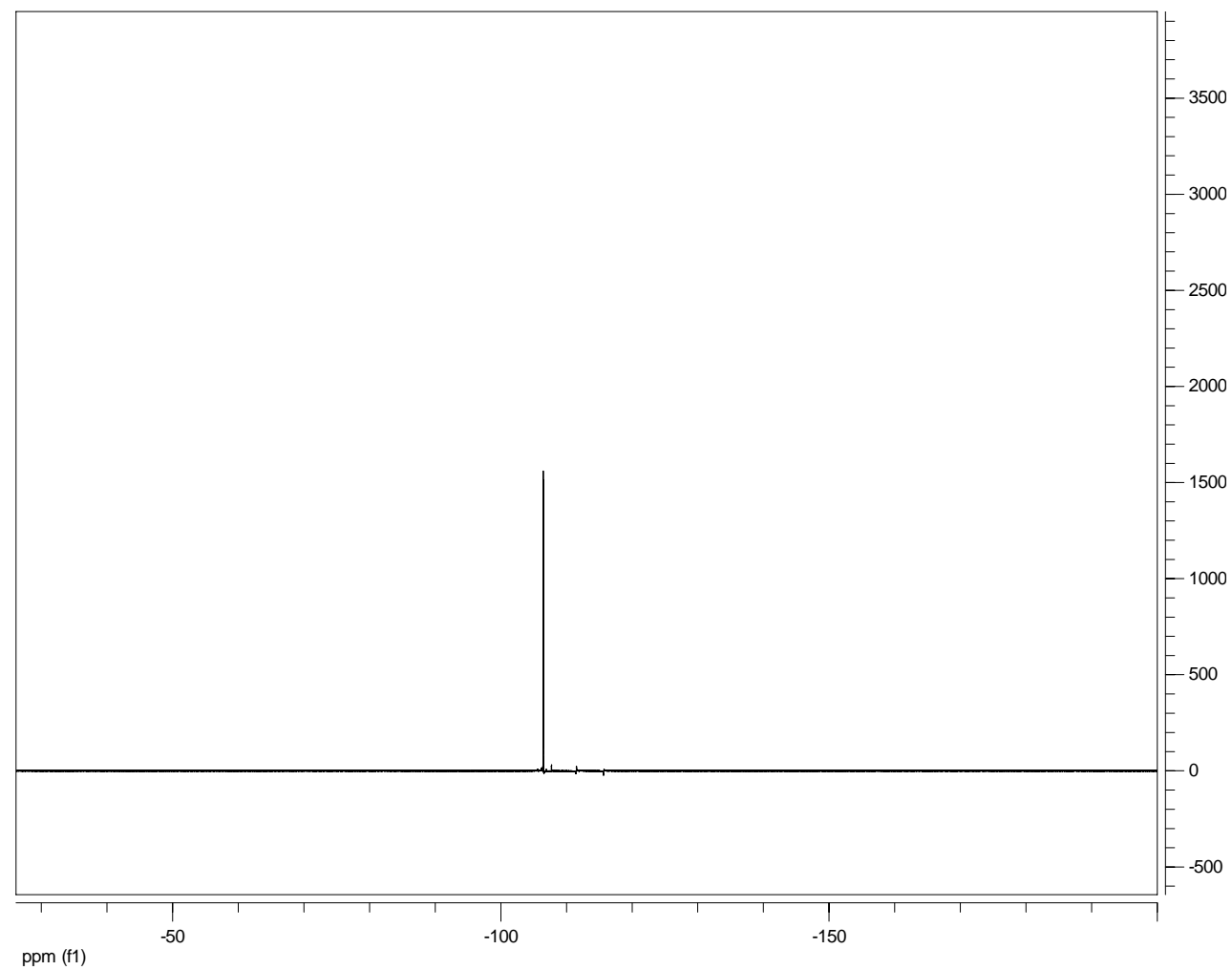


2-Methoxy-6-(3-phenyl-propyl)-benzonitrile (53)
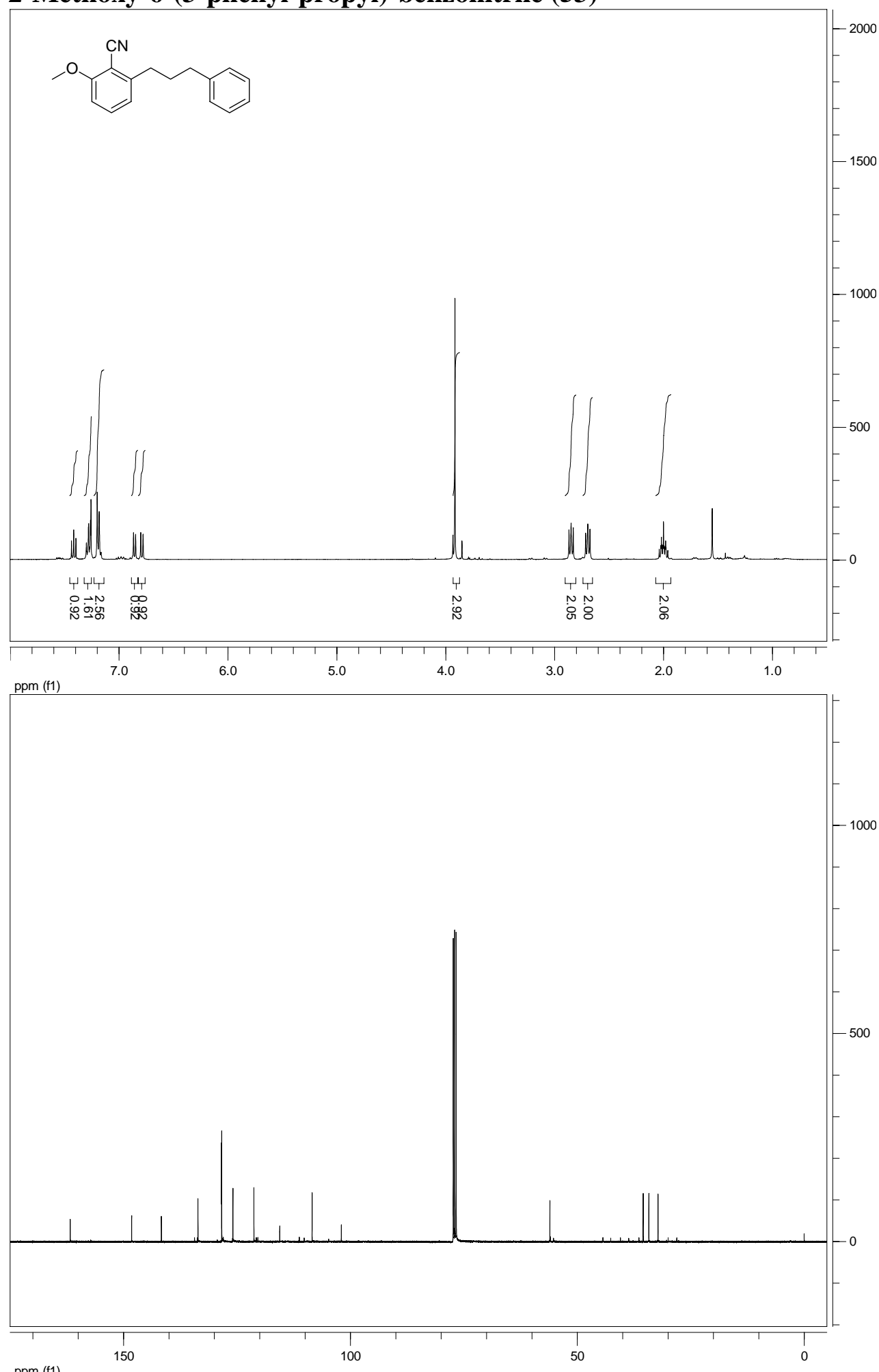

ppm (f1) 
2-Dimethylamino-6-(3-phenyl-propyl)-benzonitrile (54)

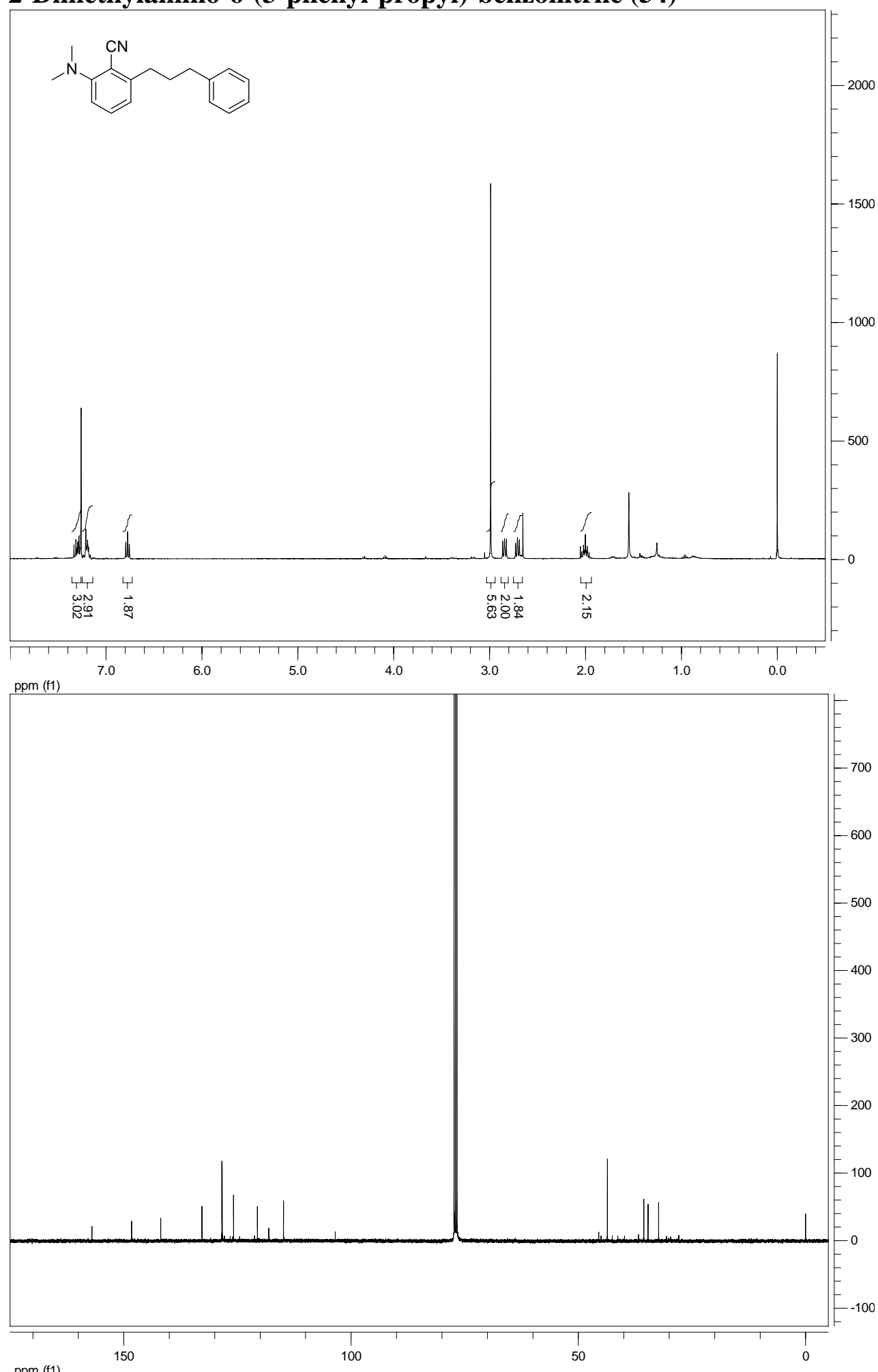

ppm (f1) 
N-[4-Cyano-3,5-bis-(3-phenyl-propyl)-phenyl]-4,N-dimethyl-benzenesulfonamide (55)
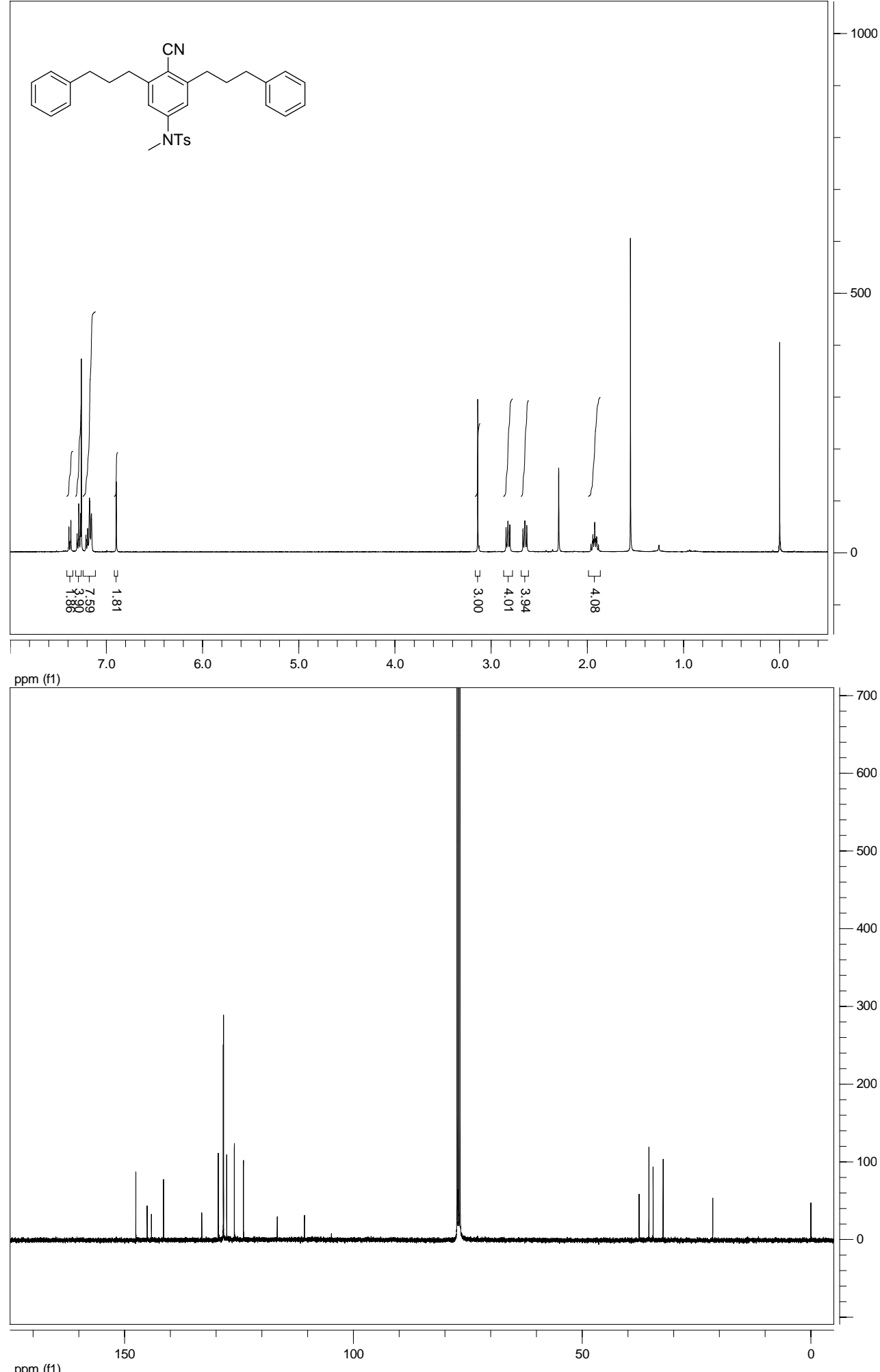
2-(4-Trifluoromethyl-phenyl)-naphthalene-1-carbonitrile (56)

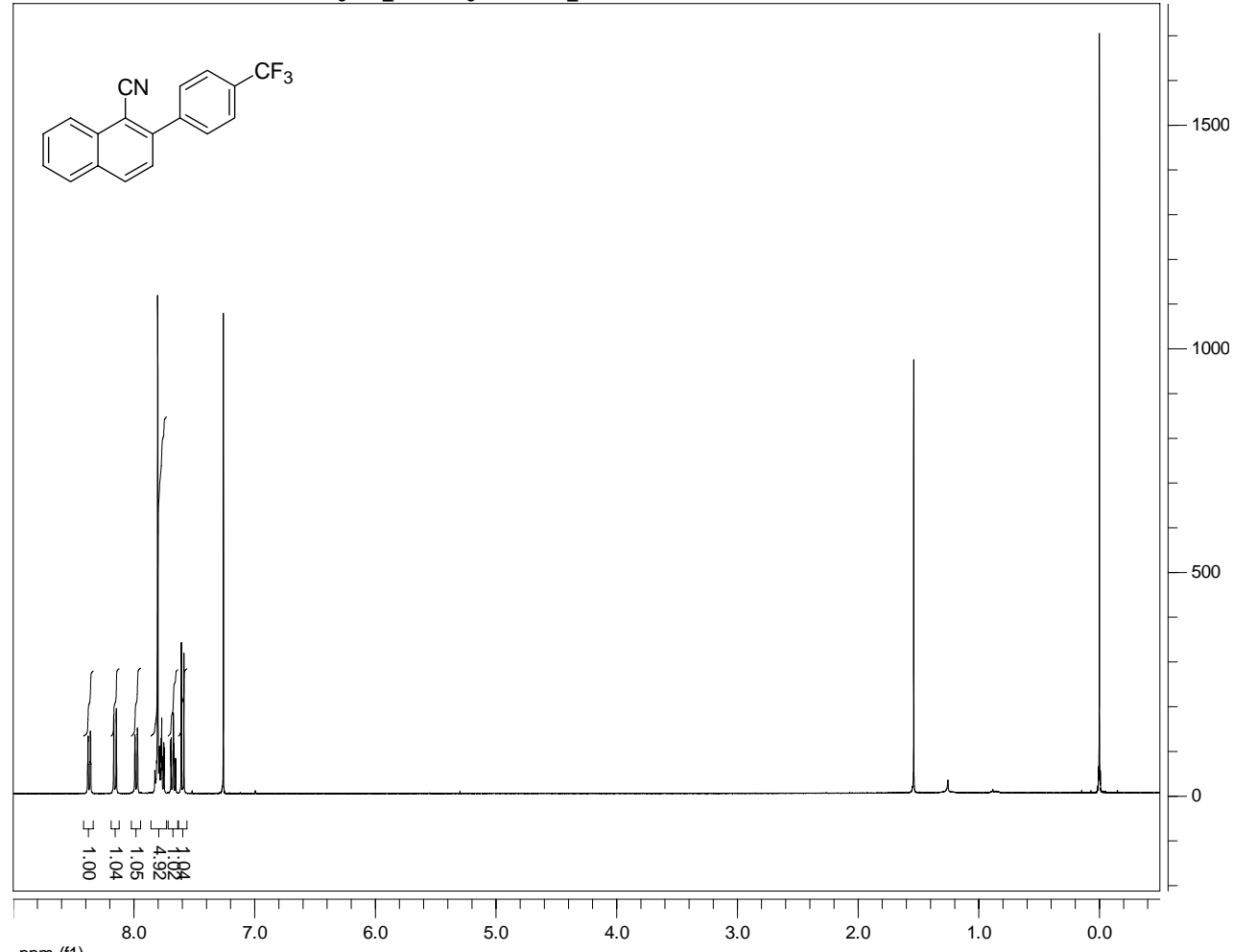

ppm (f1)

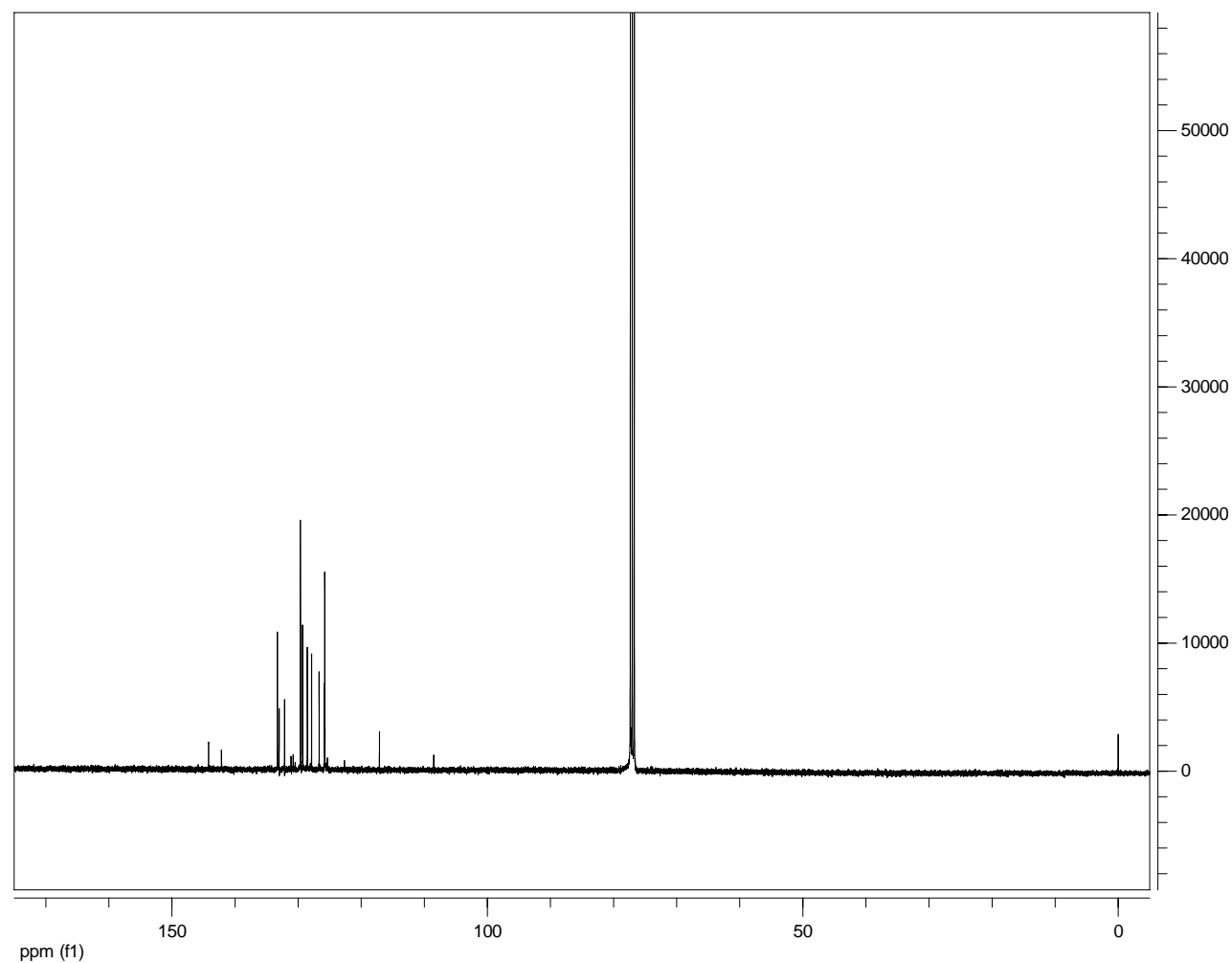


S53

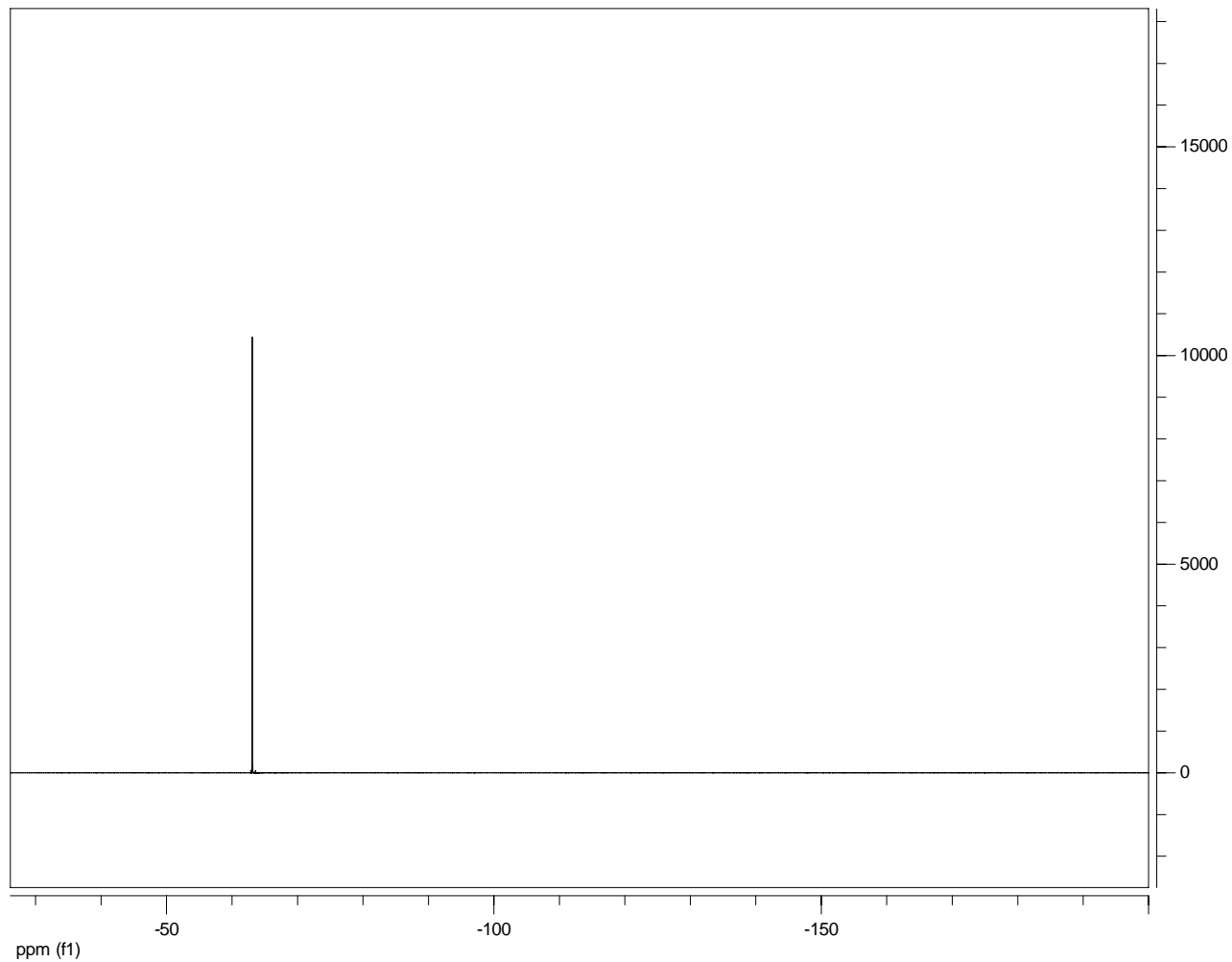


2-(3-Trifluoromethyl-phenyl)-naphthalene-1-carbonitrile (57)
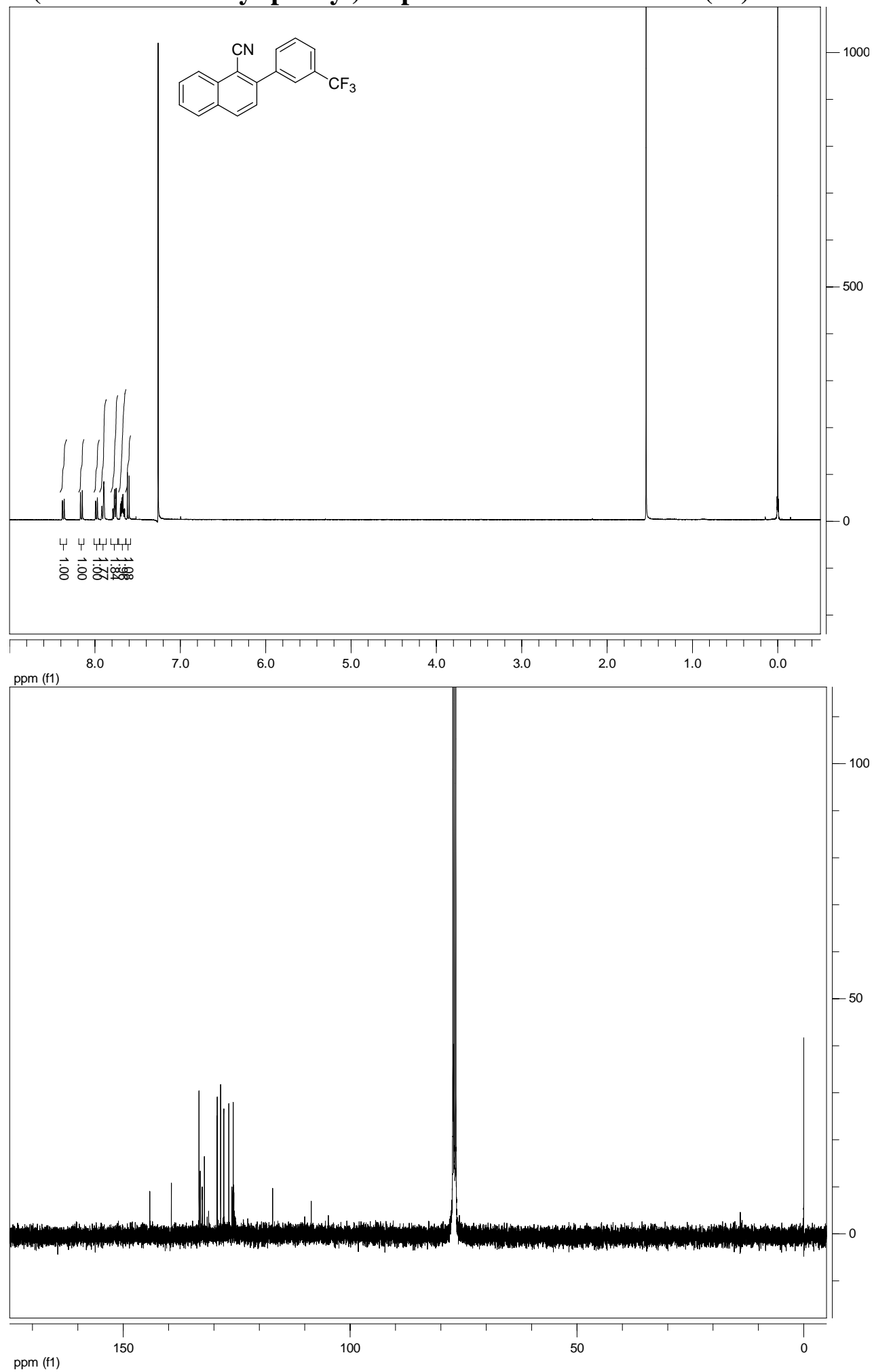


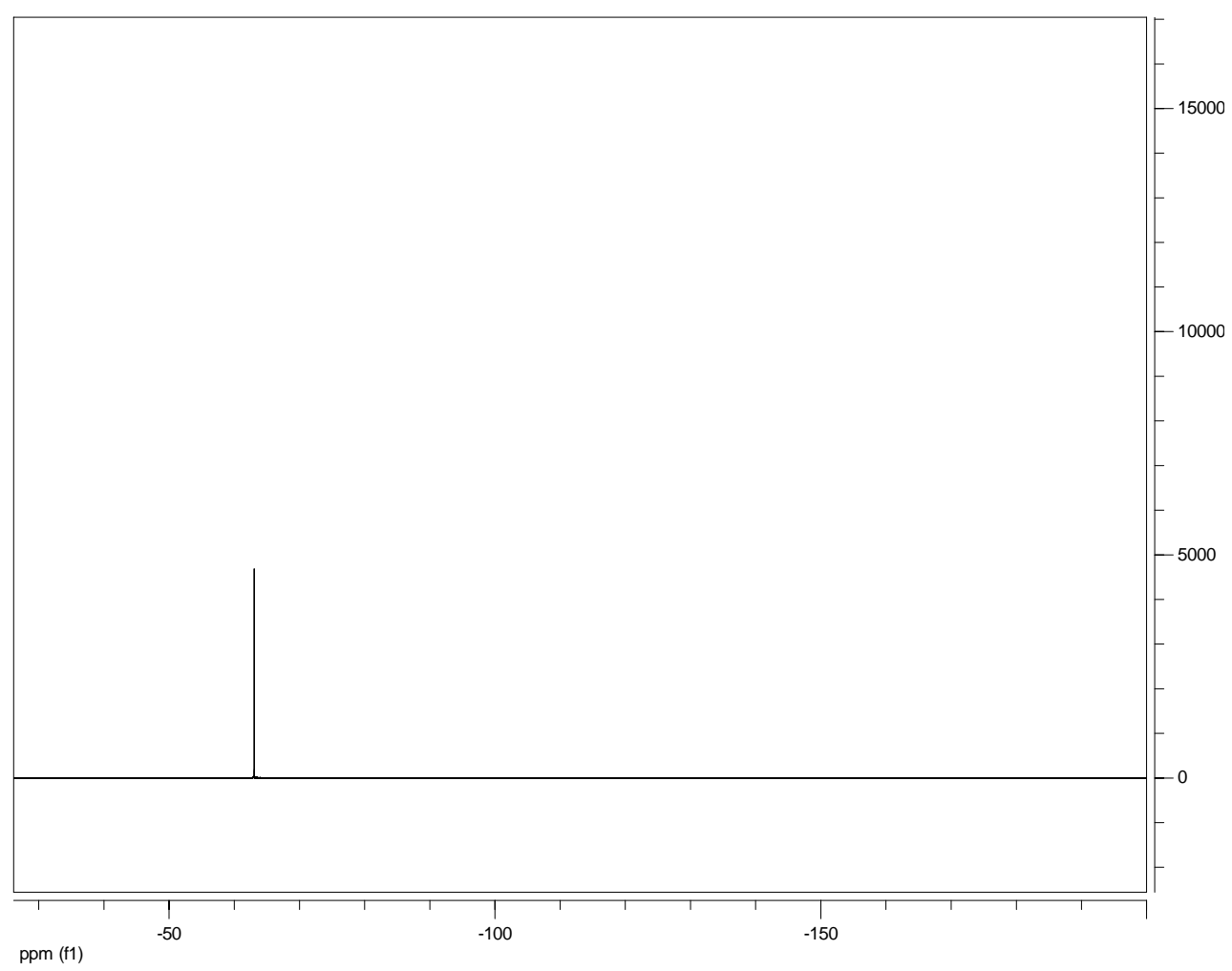


2-(4-Methanesulfonyl-phenyl)-naphthalene-1-carbonitrile (58)

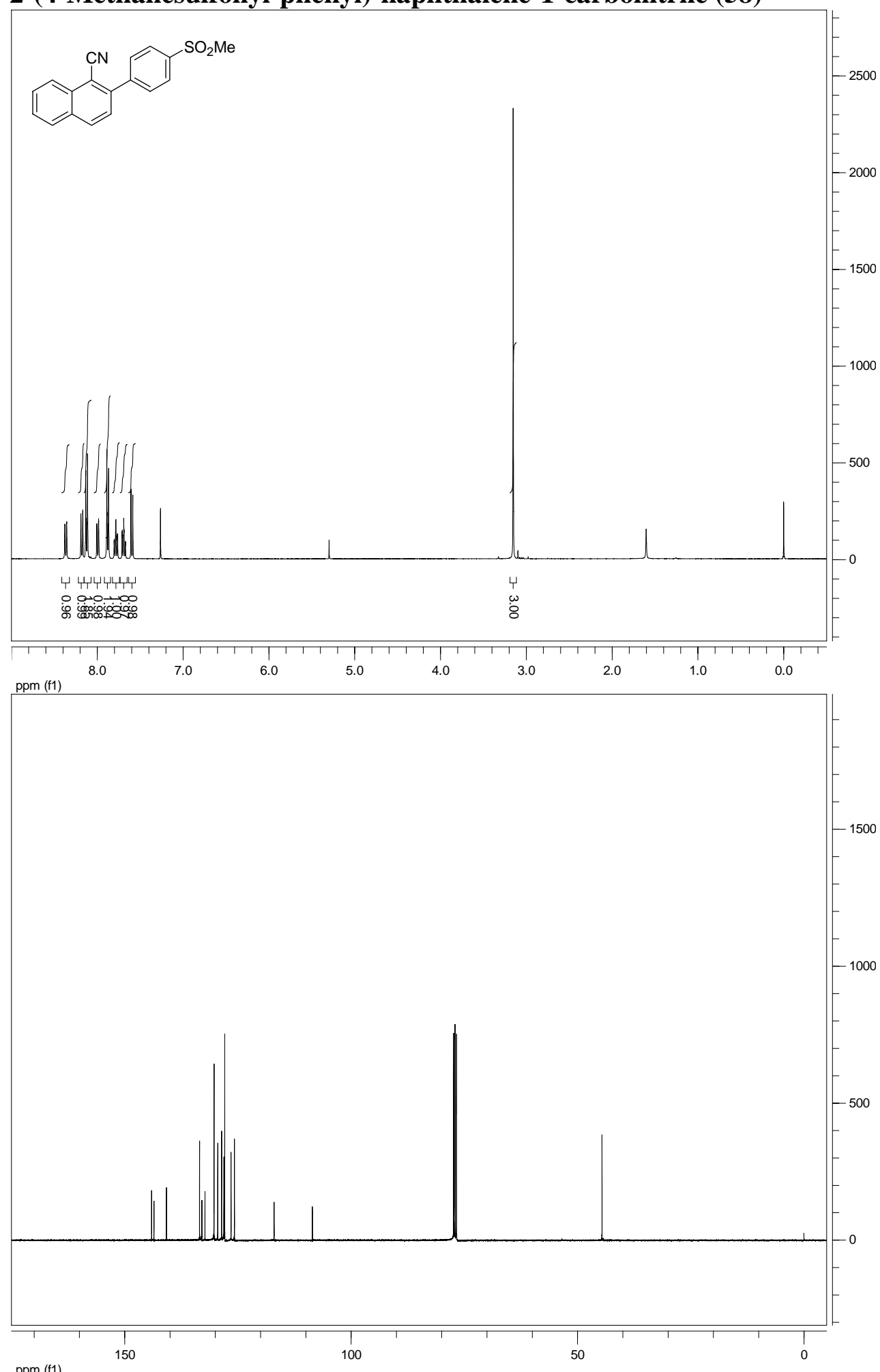

ppm (f1) 
2-(3-Methanesulfonyl-phenyl)-naphthalene-1-carbonitrile (59)
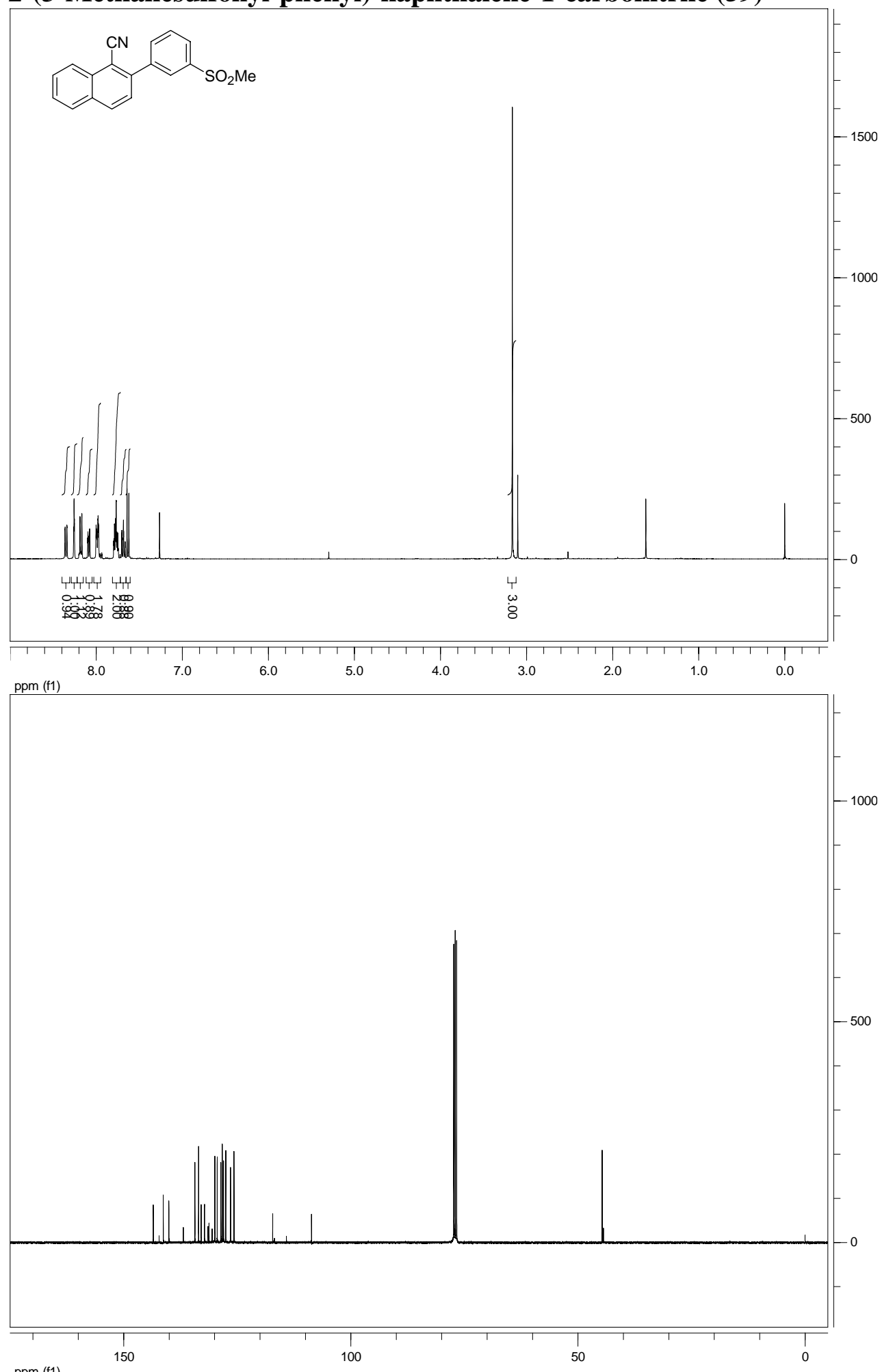

ppm (f1) 
2-(4-Formyl-phenyl)-naphthalene-1-carbonitrile (60)

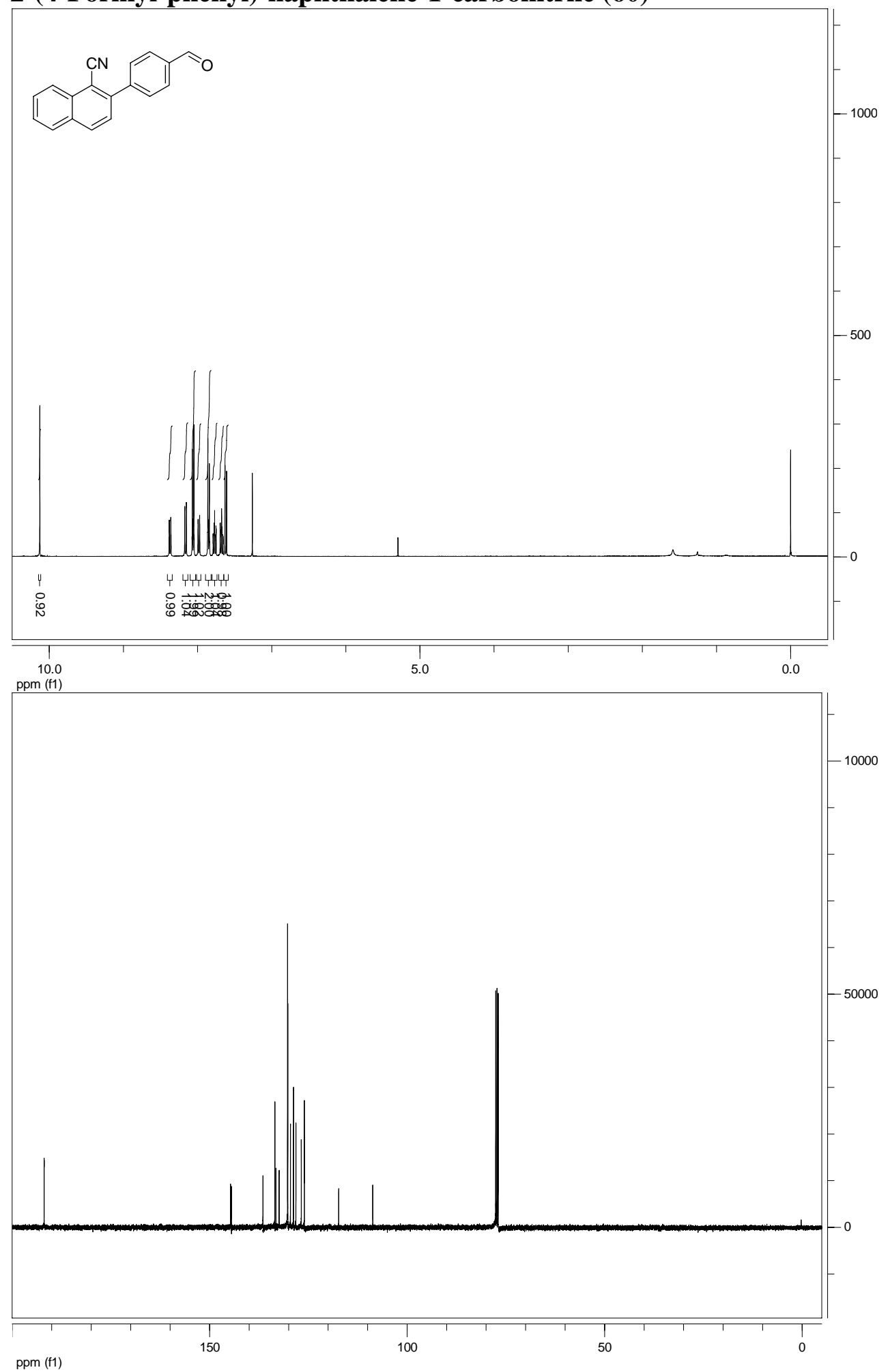


2-(3-Formyl-phenyl)-naphthalene-1-carbonitrile (61)

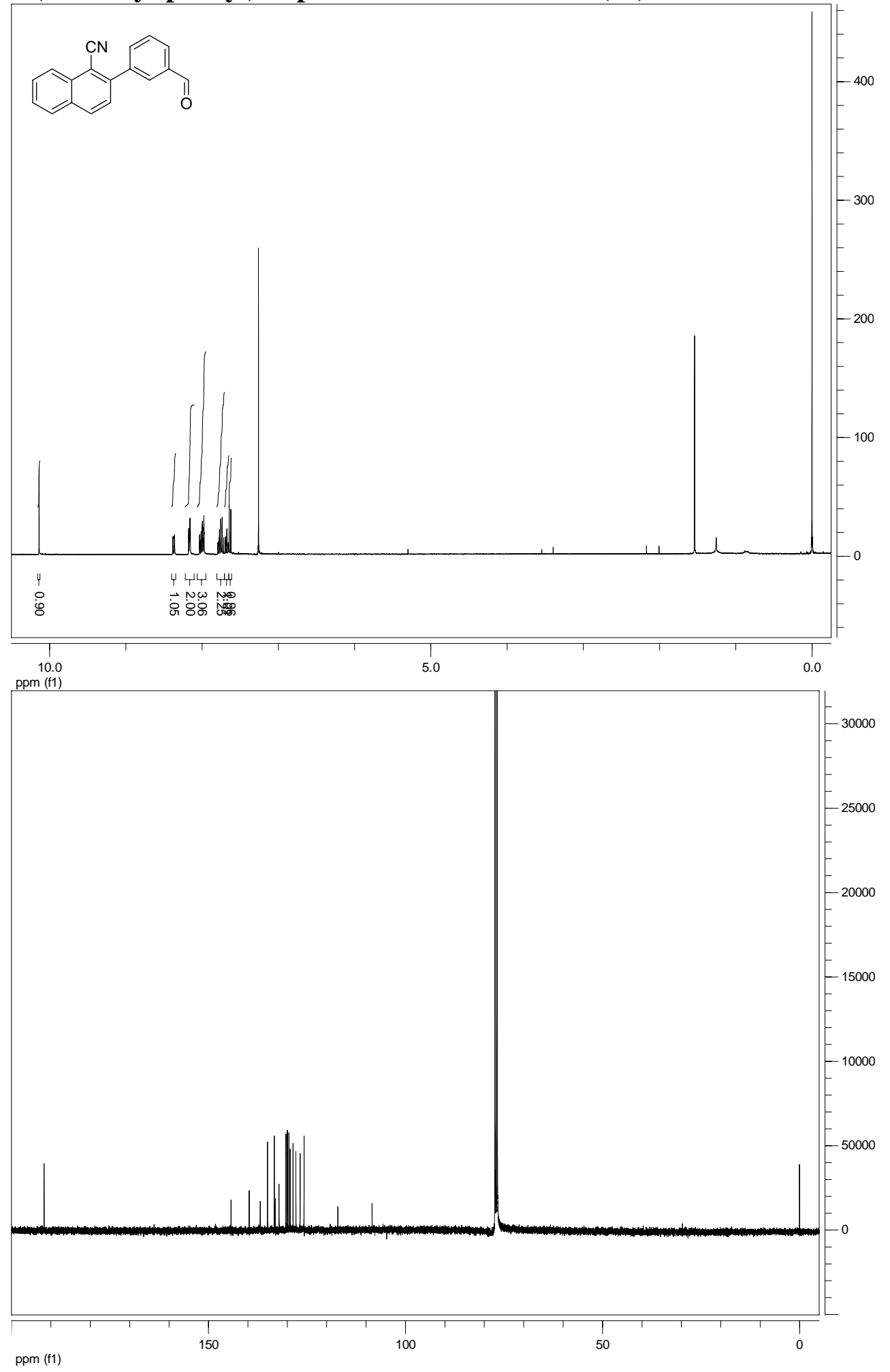


2-(3-Methoxy-phenyl)-naphthalene-1-carbonitrile (63)

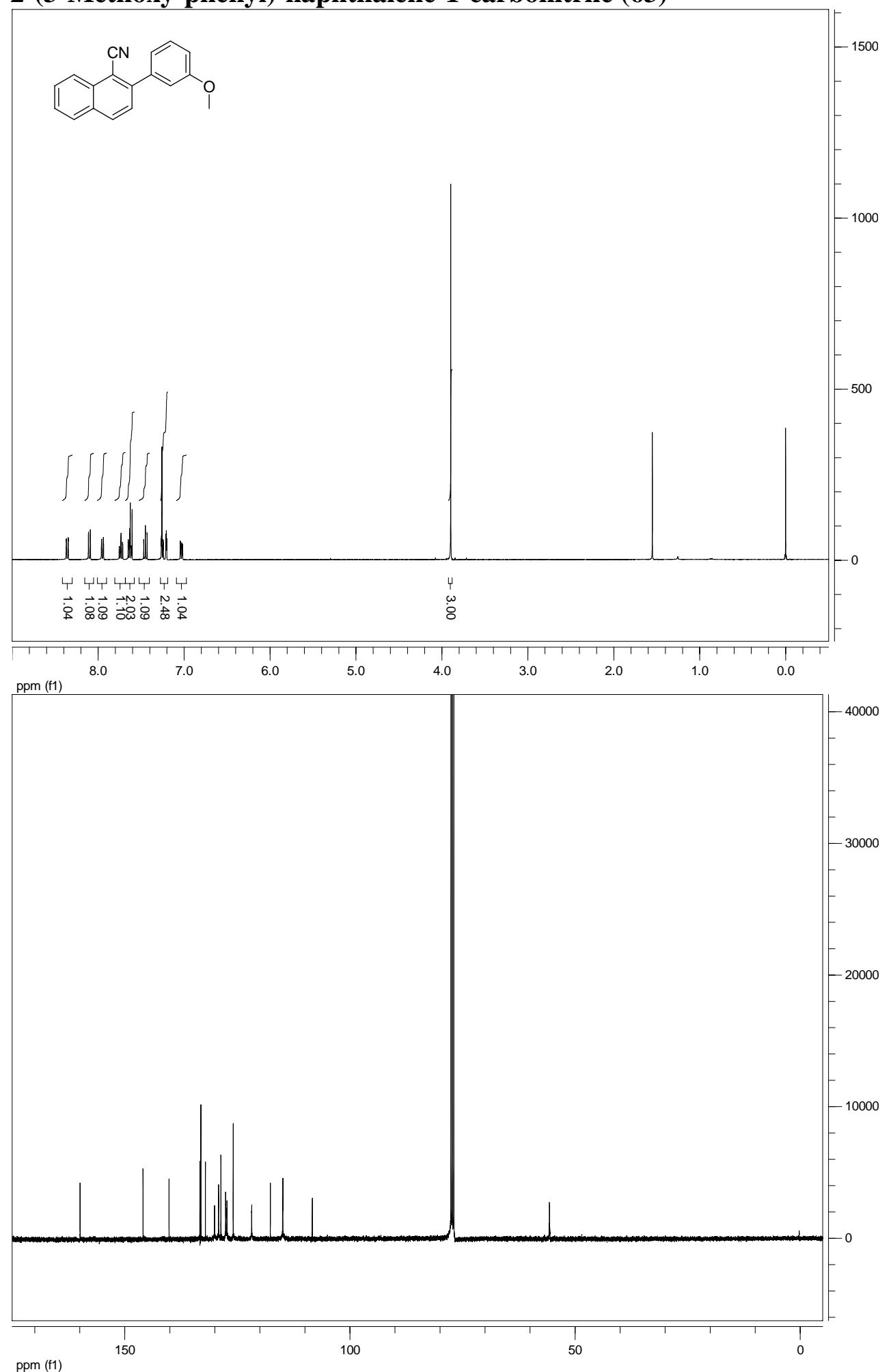


4-(1-Cyano-naphthalen-2-yl)-benzoic acid methyl ester (64)
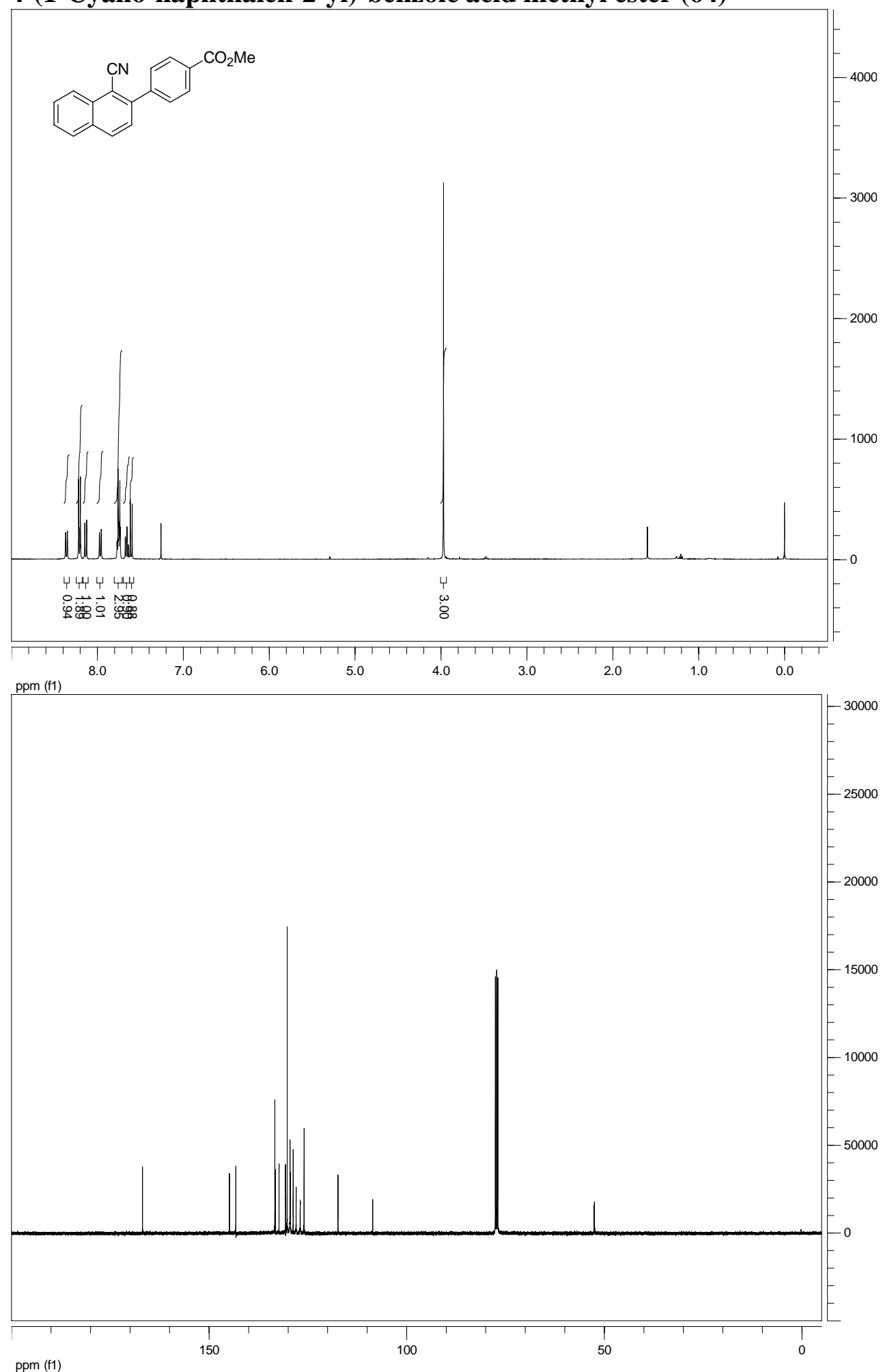
2-(1-Cyano-naphthalen-2-yl)-benzoic acid methyl ester (65)
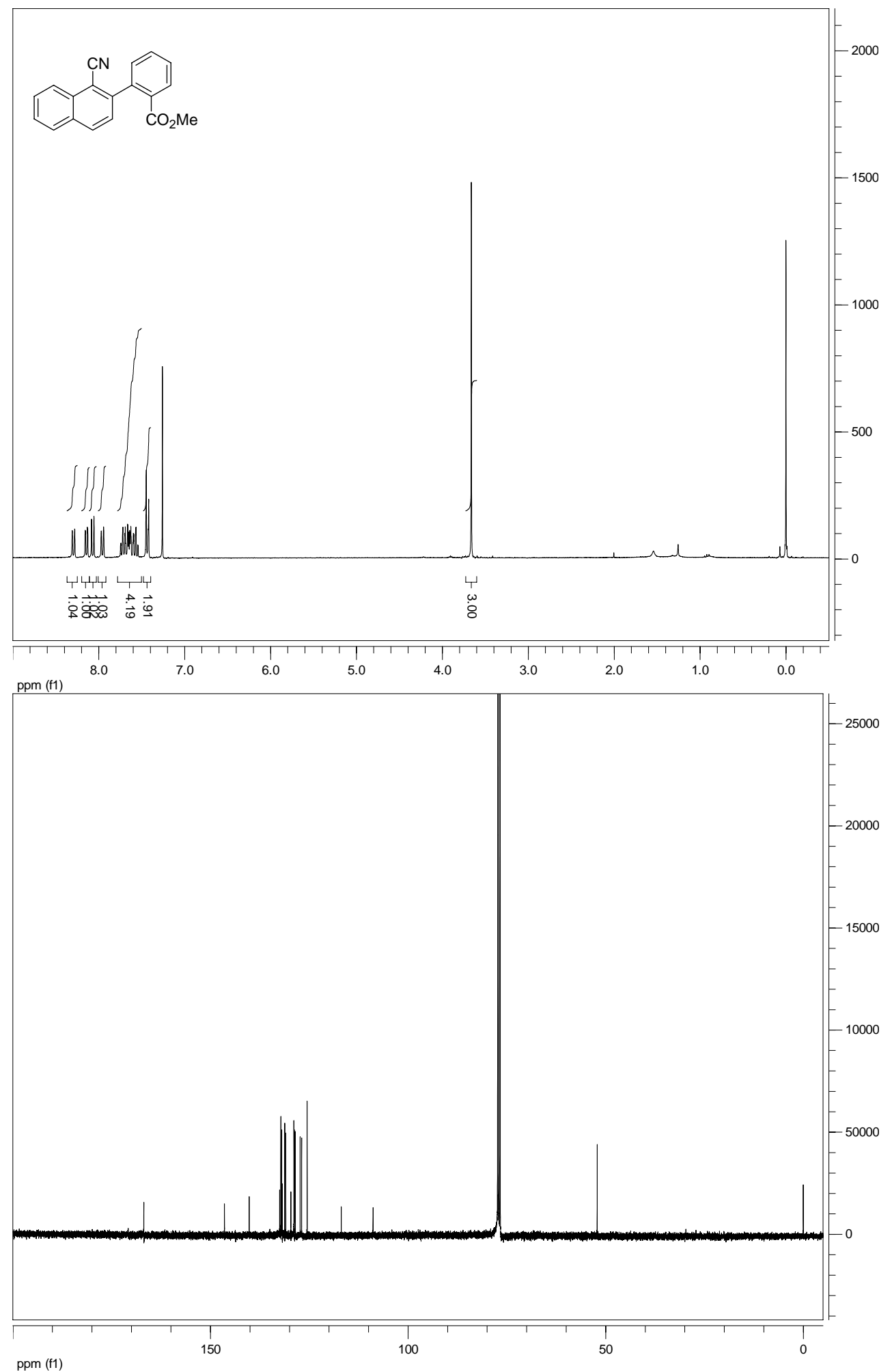
2-(2-Hydroxy-phenyl)-naphthalene-1-carbonitrile (66)
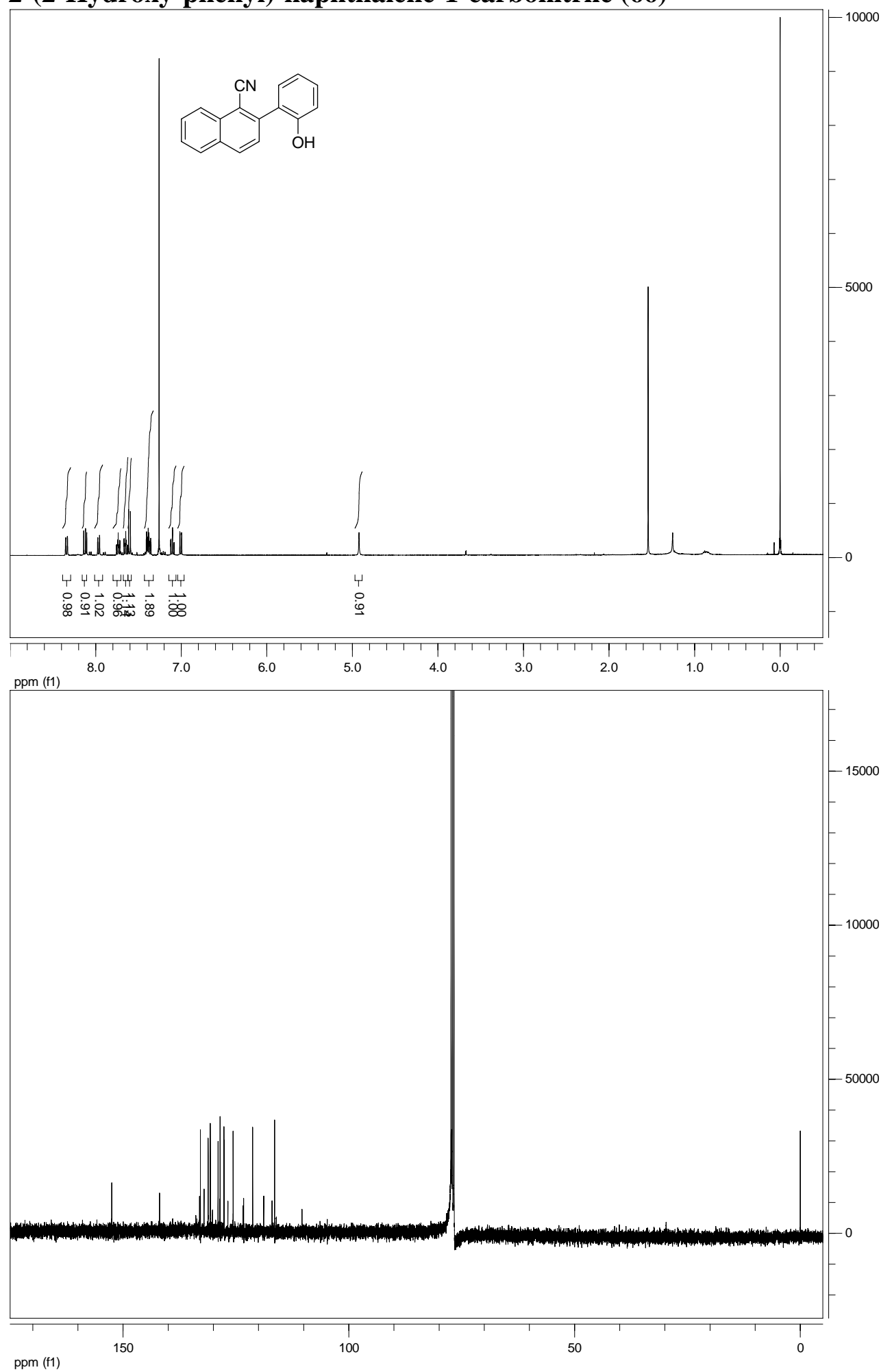
2-(4-Acetyl-phenyl)-naphthalene-1-carbonitrile (67)

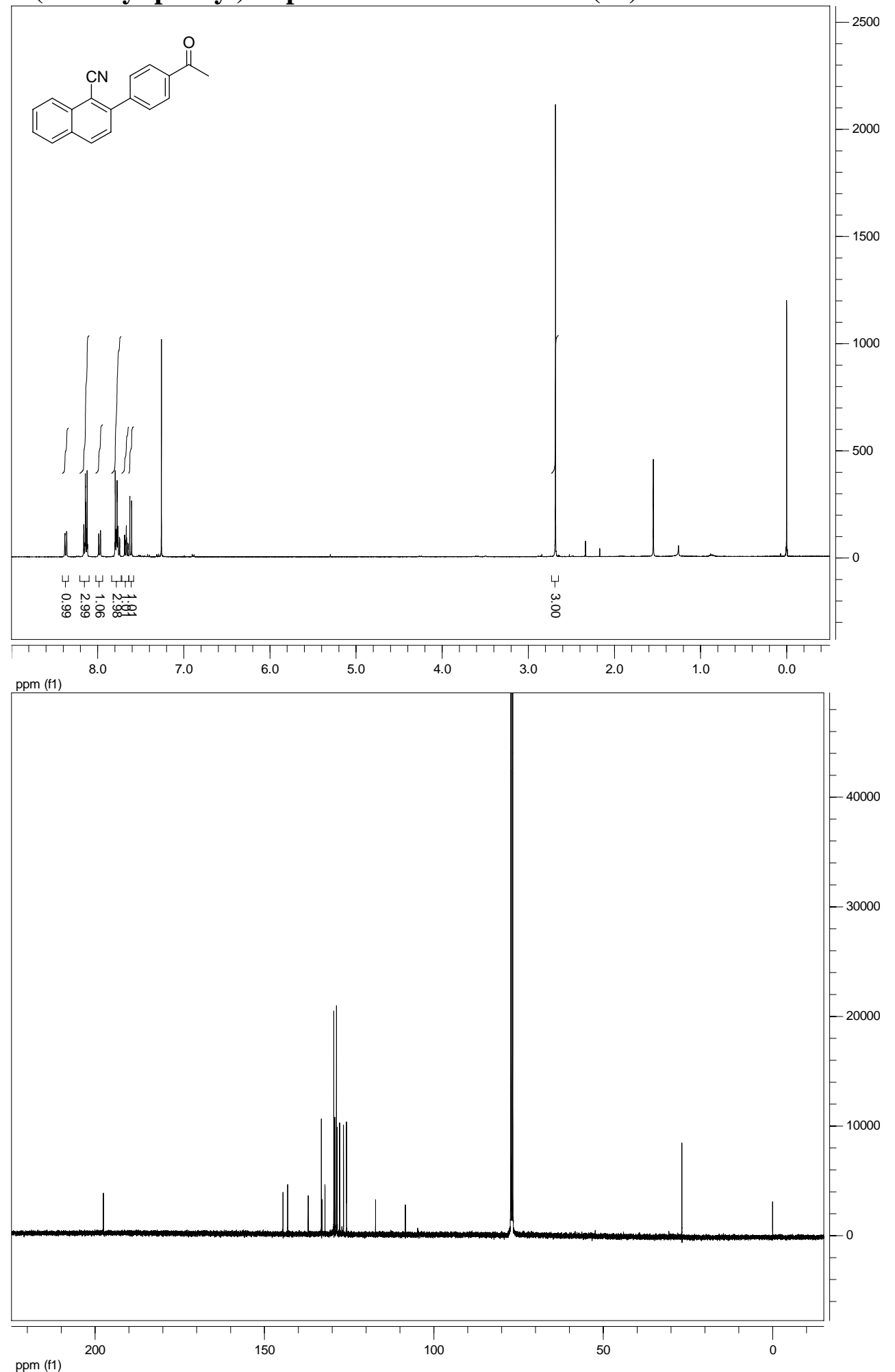


2-(4-Nitro-phenyl)-naphthalene-1-carbonitrile (68)
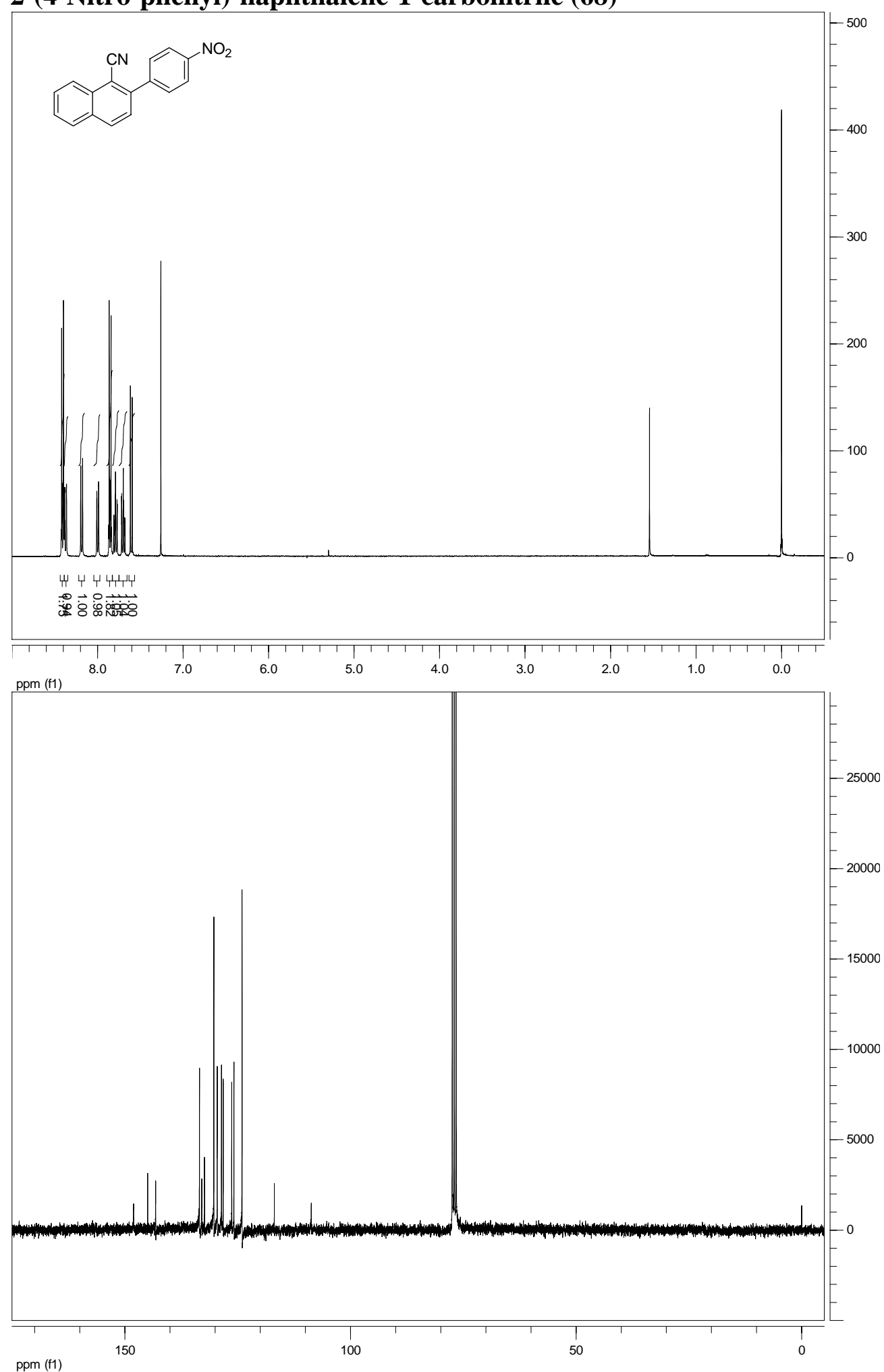
2-(4-Bromo-phenyl)-naphthalene-1-carbonitrile (69)

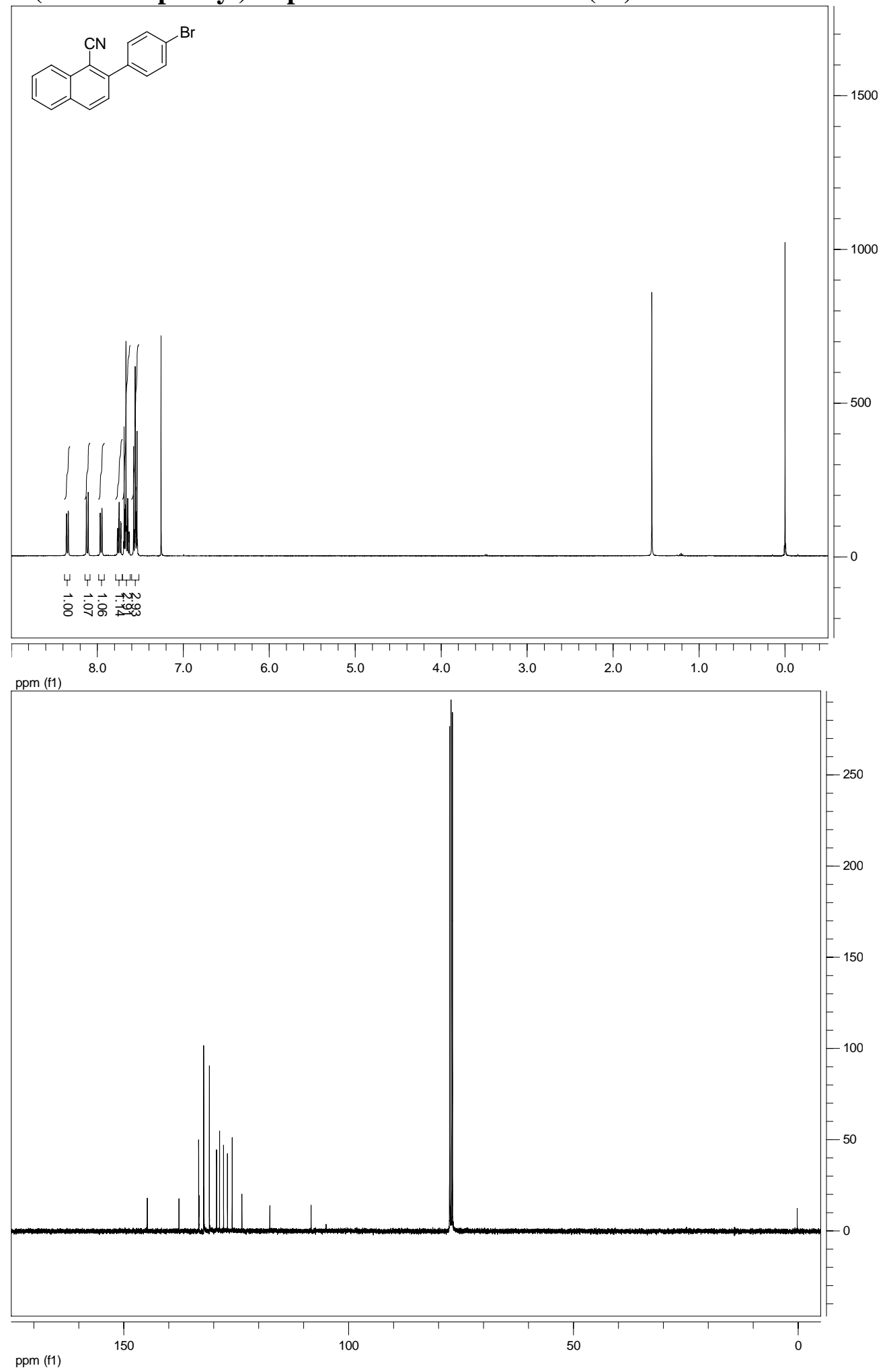


4'-Methanesulfonyl-3-methyl-biphenyl-2-carbonitrile (70)
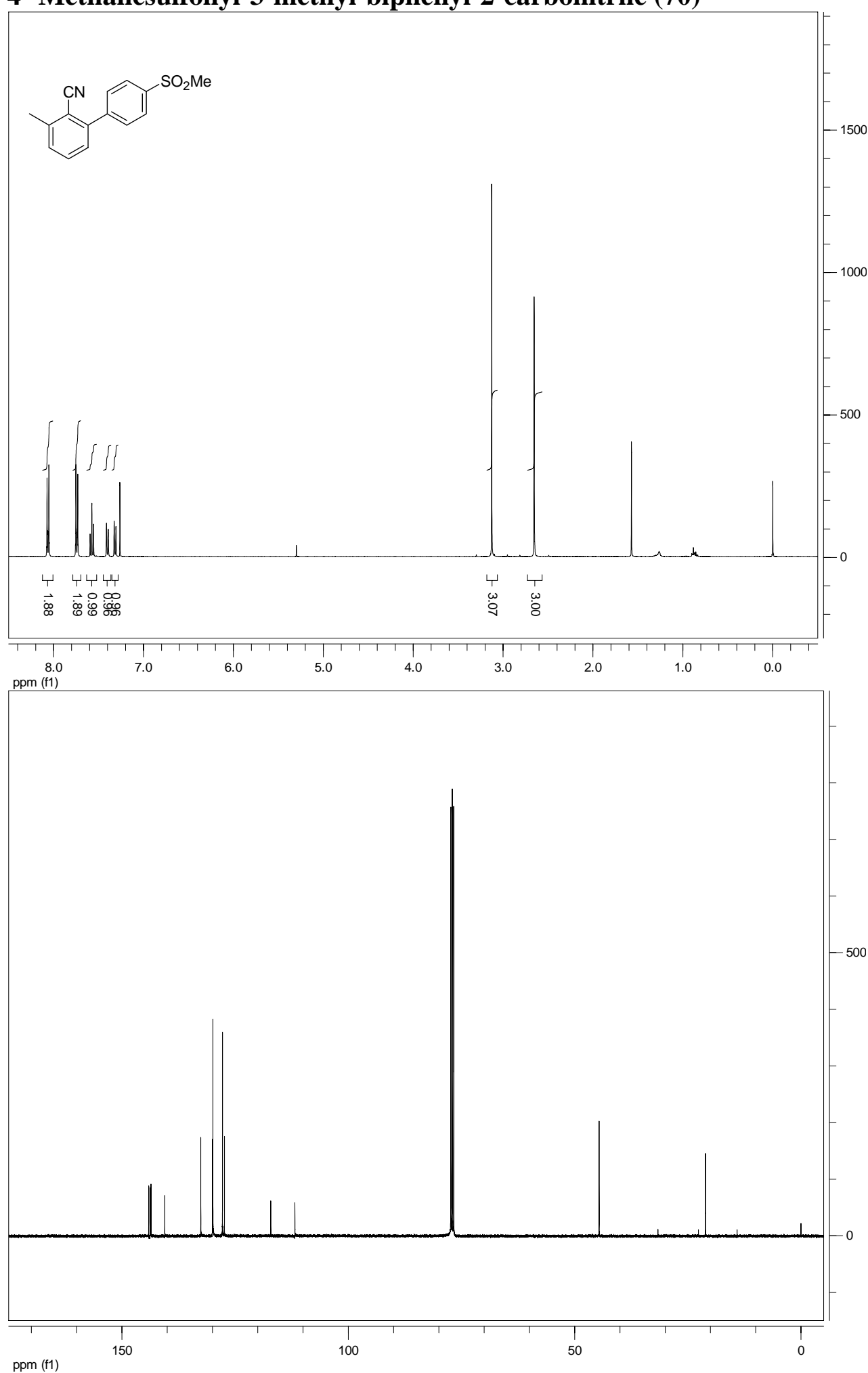
3-Chloro-4'-methanesulfonyl-biphenyl-2-carbonitrile (71)

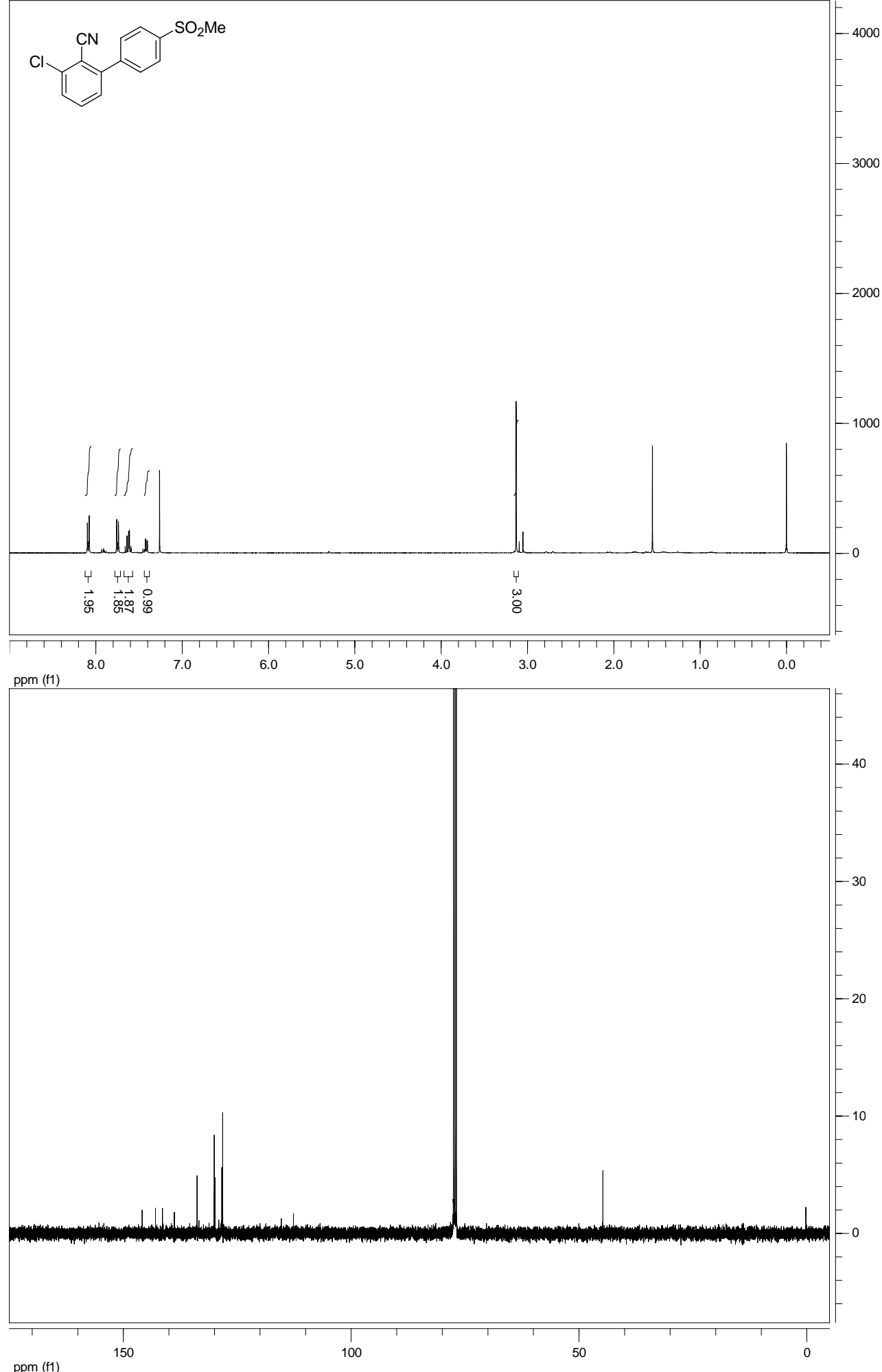

ppm (f1) 
4'-Methanesulfonyl-3-methoxy-biphenyl-2-carbonitrile (72)

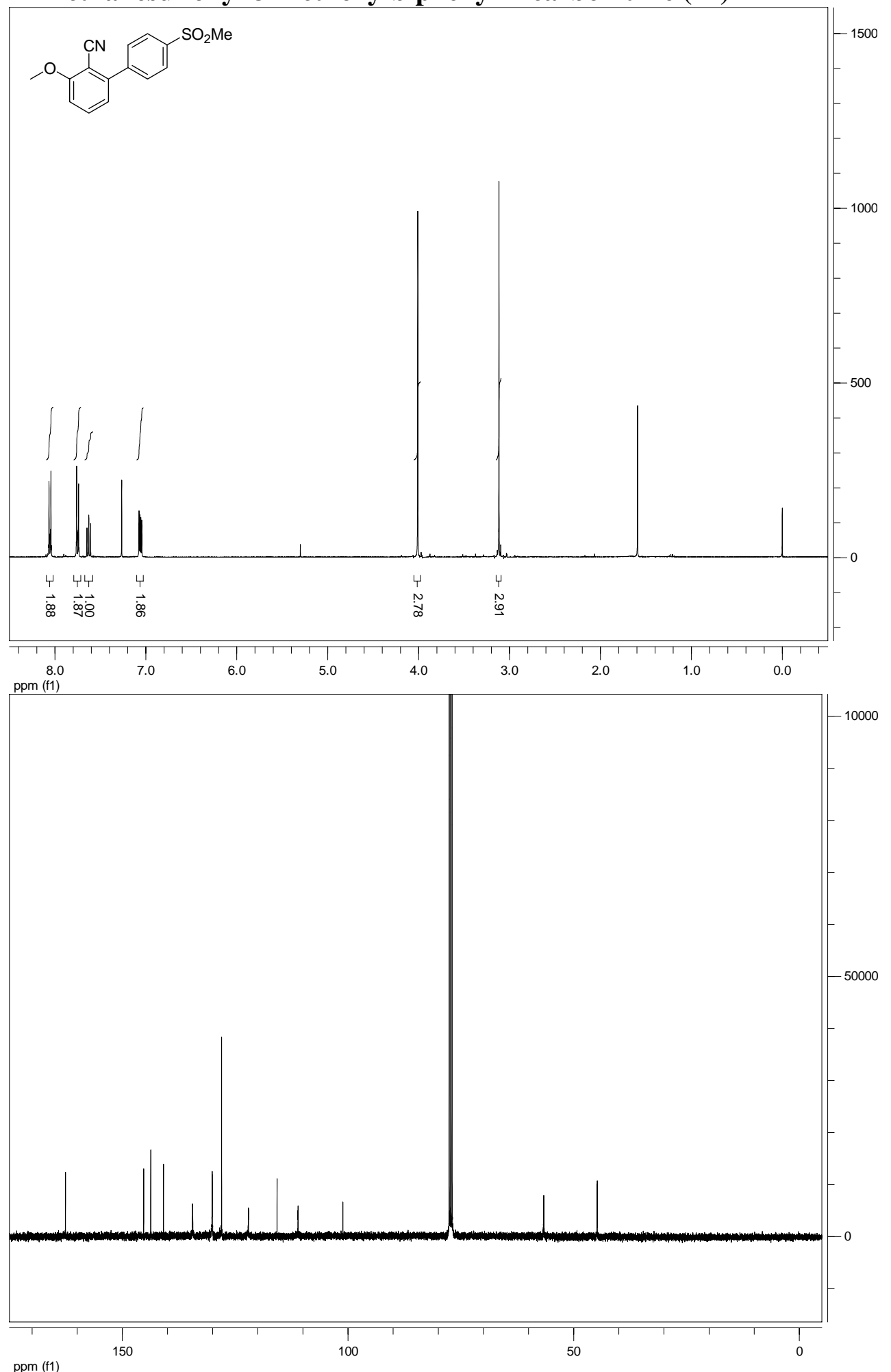


4-Chloro-4'-methanesulfonyl-3-methyl-biphenyl-2-carbonitrile (73)

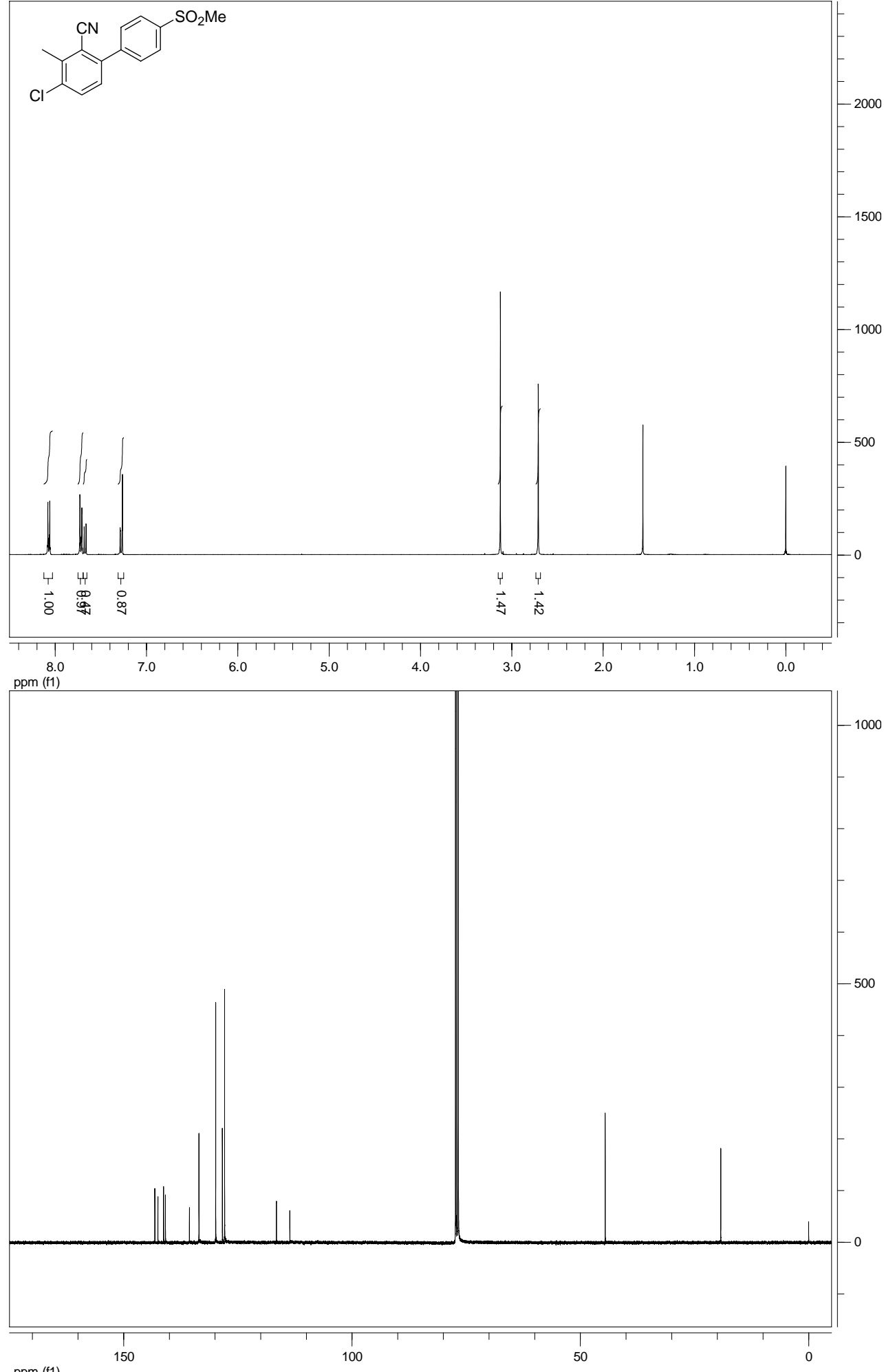

ppm (f1) 